quatrième série-tome 43 fascicule 3 mai-juin 2010

$$
\begin{aligned}
& \text { ANNALES } \\
& \text { SCIENTIFIQUES } \\
& \text { de } \\
& \text { L'ECOLE } \\
& \text { NORMALE } \\
& \text { SUPERIEURE }
\end{aligned}
$$

Aurélien DJAMENT \& Christine VESPA

Sur l' bomologie des groupes orthogonaux et symplectiques à coefficients tordus 


\title{
SUR L'HOMOLOGIE DES GROUPES ORTHOGONAUX ET SYMPLECTIQUES À COEFFICIENTS TORDUS
}

\author{
PAR Aurélien DJAMENT et Christine VESPA
}

RÉSUmÉ. - On calcule dans cet article l'homologie stable des groupes orthogonaux et symplectiques sur un corps fini $k$ à coefficients tordus par un endofoncteur usuel $F$ des $k$-espaces vectoriels (puissance extérieure, symétrique, divisée...). Par homologie stable, on entend, pour tout entier naturel $i$, les colimites des espaces vectoriels $H_{i}\left(O_{n, n}(k) ; F\left(k^{2 n}\right)\right)$ et $H_{i}\left(\operatorname{Sp}_{2 n}(k) ; F\left(k^{2 n}\right)\right)$ - dans cette situation, la stabilisation (avec une borne explicite en fonction de $i$ et $F$ ) est un résultat classique de Charney.

Tout d'abord, nous donnons un cadre formel pour relier l'homologie stable de certaines suites de groupes à l'homologie de petites catégories convenables, à l'aide d'une suite spectrale, qui dégénère dans de nombreux cas favorables. Cela nous permet d'ailleurs de retrouver des résultats de Betley sur l'homologie stable des groupes linéaires et des groupes symétriques, par des méthodes purement algébriques (sans recours à la $K$-théorie stable).

Pour une application exploitable de ce formalisme aux groupes orthogonaux ou symplectiques sur un corps fini, nous réinterprétons la deuxième page de notre suite spectrale en termes de foncteurs de Mackey non additifs et utilisons leurs propriétés d'acyclicité. Cela permet d'obtenir une simplification spectaculaire de la deuxième page de la suite spectrale en employant de puissants résultats d'annulation connus en homologie des foncteurs.

Dans le cas où les groupes orthogonaux ou symplectiques sont pris sur un corps fini et les coefficients à valeurs dans les espaces vectoriels sur ce même corps, nous pouvons mener le calcul de cette deuxième page grâce à des résultats classiques : annulation homologique à coefficients triviaux (Quillen, Fiedorowicz-Priddy), et calcul des groupes de torsion entre foncteurs usuels (Franjou-FriedlanderScorichenko-Suslin, Chałupnik). Ceci permet de nombreux calculs d'homologie stable à coefficients.

Abstract. - We compute the stable homology of orthogonal and symplectic groups, over a finite field $k$, when the coefficients module is twisted by a usual endofunctor $F$ of $k$-vector spaces (e.g. an exterior, a symmetric, or a divided power) - that is, for each natural integer $i$, we compute the colimit of the vector spaces $H_{i}\left(O_{n, n}(k) ; F\left(k^{2 n}\right)\right)$ and $H_{i}\left(\mathrm{Sp}_{2 n}(k) ; F\left(k^{2 n}\right)\right)$. Stabilization in this situation is a classical result of Charney.

We first set a formal framework, within which the stable homology of some families of groups relates through a spectral sequence to the homology of suitable small categories. The spectral sequence

Les auteurs ont bénéficié des contrats INTAS 06-1000017-8609 et ANR BLAN08-2-338236 (HGRT : Nouveaux liens entre la théorie de l'homotopie et la théorie des groupes et des représentations). 
collapses in many cases. We illustrate this purely algebraic method to retrieve results of Betley for the stable homology of the general linear groups and of the symmetric groups.

We then apply our approach to orthogonal and symplectic groups over a finite field. To this end, we reinterpret the second page of our spectral sequence with Mackey functors and use their acyclicity properties. It allows us to simplify the second page of the spectral sequence, by using powerful cancellation results for functor homology.

For the orthogonal as for the symplectic groups over a finite field, and for coefficients modules over the same field, we compute the second page of the spectral sequence. Classical results prove useful at this point: homological cancellation with trivial coefficients (Quillen, Fiedorowicz-Priddy), and calculation of the torsion groups between usual functors (Franjou-Friedlander-Scorichenko-Suslin, Chałupnik). This provides extensive computations of stable homology with coefficients.

\section{Introduction}

Cet article a pour objet l'homologie stable à coefficients tordus de familles de groupes classiques, c'est-à-dire la colimite de leur homologie; celle-ci, dans de nombreux cas, est atteinte en temps fini pour chaque degré. Alors que cette homologie stable possède un comportement plus régulier que l'homologie instable, elle s'avère généralement inaccessible au calcul direct. Depuis les travaux de Betley ([4]) et Suslin ([14]), on dispose cependant d'une interprétation de l'homologie stable des groupes linéaires en terme d'homologie des foncteurs, qui permet de mener à bien de nombreux calculs. Néanmoins aucun analogue n'était jusqu'alors connu dans les cas des groupes orthogonaux et symplectiques.

Le présent travail établit un isomorphisme naturel entre l'homologie stable des groupes orthogonaux (ou symplectiques) sur un corps fini, à coefficients tordus par un foncteur polynomial, et des groupes de torsion entre endofoncteurs des espaces vectoriels. Il est remarquable que cet isomorphisme ne fasse pas intervenir de catégorie d'espaces quadratiques (ou symplectiques), et qu'il ne s'exprime que par la catégorie déjà bien étudiée des endofoncteurs entre espaces vectoriels. Cela rend calculables les groupes d'homologie stable des groupes orthogonaux pour les foncteurs polynomiaux usuels : puissances symétriques, extérieures, tensorielles, etc.

L'homologie stable à coefficients constants des groupes orthogonaux sur un corps fini $k$ a été calculée dans les années 1970 par Fiedorowicz et Priddy [12], en généralisant les méthodes initiées par Quillen [25] pour les groupes linéaires. L'homologie stable à coefficients dans un corps de même caractéristique que $k$ est triviale pour les groupes orthogonaux (si la caractéristique de $k$ est impaire), symplectiques ou linéaires sur $k$. De plus, on dispose de résultats de stabilité homologique pour les familles de groupes classiques sur les corps finis, à coefficients constants ou tordus par un foncteur polynomial - le cas des groupes orthogonaux étant dû à Charney [8]. Pour autant, la détermination de cette valeur stable semblait jusqu'à présent inabordable, y compris pour des coefficients tordus par un foncteur polynomial non constant élémentaire.

L'annulation de l'homologie stable du groupe linéaire sur un anneau, à coefficients tordus par un foncteur polynomial sans terme constant, a été obtenue par Betley [3] par des méthodes complètement différentes de celles utilisées pour les coefficients constants. En 1999, 
Betley [4] et Suslin [14, appendice] ont démontré indépendamment une généralisation du résultat précédent, pour des coefficients tordus par un bifoncteur, polynomial en chaque variable. L'homologie stable n'est alors plus généralement nulle, mais naturellement isomorphe à l'homologie de Hochschild de la catégorie des $k$-espaces vectoriels de dimension finie, à coefficients dans le bifoncteur. Ces groupes d'homologie sont accessibles pour les foncteurs usuels, comme l'a notamment montré l'article [14]. La démonstration de Betley repose sur un analogue, en termes de groupes algébriques, du lien entre l'homologie des groupes linéaires et l'homologie de Hochschild des bifoncteurs polynomiaux, résultat établi un peu plus tôt par Friedlander et Suslin [16]. Suslin s'appuie entièrement, pour sa part, sur des considérations internes aux foncteurs entre espaces vectoriels. Sa démarche a été étendue peu après à l'homologie stable des groupes linéaires sur un anneau arbitraire par Scorichenko [27], qui a obtenu un isomorphisme entre $K$-théorie stable et homologie de Hochschild d'un bifoncteur polynomial.

Ces résultats constituent une illustration de la richesse du point de vue des catégories de foncteurs, dont on trouvera une synthèse dans [13]. Cette approche a déjà permis de résoudre de difficiles conjectures de finitude, comme en témoignent les travaux de Friedlander-Suslin [16], et tout récemment de Touzé et van der Kallen [31]. L'étude fine des catégories de foncteurs s'est également poursuivie avec, par exemple, les résultats cohomologiques de Chałupnik [7] et Touzé [29], complétant ceux de [14], ou l'introduction de nouvelles catégories de foncteurs reliées aux groupes orthogonaux dans [33].

Nous présentons maintenant le contenu de l'article. Une première section dégage un cadre formel pour étudier l'homologie stable d'une suite convenable de groupes, à partir de l'homologie d'une catégorie adaptée à la situation. Plus précisément, on considère une petite catégorie monoïdale symétrique $(\mathscr{C}, \oplus, 0)$ dont l'unité 0 est objet initial, et $A$ un objet de $\mathscr{C}$. Pour chaque entier naturel $i$, on note $G(i)$ le groupe d'automorphismes $\operatorname{Aut}_{\mathscr{C}}\left(A^{\oplus i}\right)$. La catégorie $\mathscr{C}$ sera par exemple celle des modules projectifs de type fini sur un anneau $A$, avec les monomorphismes scindés comme flèches, et la somme directe pour structure monoïdale. On peut prendre aussi pour $\mathscr{C}$ la catégorie des ensembles finis avec injections, pour $A$ un ensemble à 1 élément et la réunion disjointe pour $\oplus$, ce qui nous permettra de retrouver des résultats de Betley ([5]) sur l'homologie stable des groupes symétriques. Pour le sujet principal de cet article, on considère une catégorie $\mathscr{C}$ d'espaces quadratiques, ou symplectiques, de dimension finie sur un corps commutatif, pour $A$ un espace hyperbolique ou symplectique de dimension 2 et pour $\oplus$ la somme orthogonale. Ces exemples sont détaillés au paragraphe 1.2.

Dans le cas général, la suite de morphismes :

$$
0 \rightarrow A \rightarrow \cdots \rightarrow A^{\oplus n} \rightarrow A^{\oplus(n+1)} \rightarrow \cdots
$$

donnés par :

$$
A^{\oplus n} \simeq A^{\oplus n} \oplus 0 \stackrel{\operatorname{Id} \oplus(0 \rightarrow A)}{\longrightarrow} A^{\oplus n} \oplus A \simeq A^{\oplus(n+1)}
$$

est compatible aux actions des groupes d'automorphismes $G(n)=\operatorname{Aut}\left(A^{\oplus n}\right)$, où $G(n)$ agit sur $A^{\oplus(n+1)}$ via le morphisme :

$$
G(n) \stackrel{g \mapsto g \oplus A}{\longrightarrow} G(n+1)
$$


Ceci induit une suite naturelle de morphismes :

$$
\cdots \rightarrow H_{*}\left(G(n) ; F\left(A^{\oplus n}\right)\right) \rightarrow H_{*}\left(G(n+1) ; F\left(A^{\oplus(n+1)}\right)\right) \rightarrow \cdots,
$$

où $F$ est un foncteur de $\mathscr{C}$ vers les groupes abéliens. On appelle homologie stable des groupes $G(n)$ à coefficients dans $F$ la colimite de cette suite.

Il existe toujours un morphisme naturel de l'homologie stable des groupes $G(n)$ à coefficients dans $F$ vers l'homologie de $\mathscr{C}$ à coefficients dans $F$. Sous de bonnes hypothèses sur la catégorie $\mathscr{C}$, ce morphisme est un isomorphisme en degré 0 , mais ce n'est plus en général le cas au-delà, où l'on obtient, au paragraphe 2.2, une suite spectrale convergeant vers cette homologie stable et dont la deuxième page s'exprime par des groupes de torsion sur la catégorie $\mathscr{C}$.

Cette suite spectrale offre une alternative purement algébrique à la suite spectrale de la $K$-théorie stable convergeant vers l'homologie stable du groupe linéaire à coefficients tordus, et à ses avatars la généralisant aux familles de groupes usuelles. En effet, dans les cas connus où l'on sait exprimer la $K$-théorie stable en termes algébriques plus simples, et où la suite spectrale correspondante s'arrête dès la deuxième page, on observe un phénomène analogue dans notre formalisme - voir notamment nos propositions 2.22 et 2.26. Ceci n'est guère surprenant dans la mesure où les travaux de Scorichenko (cas classique de la $K$-théorie stable) ou Betley (cas des groupes symétriques) n'utilisent en fait nullement la définition de la $K$-théorie stable, mais seulement l'existence d'une suite spectrale naturelle d'un certain type, et où l'article [6] de Betley et Pirashvili identifie dans de nombreux cas la $K$-théorie stable à un foncteur dérivé défini de façon purement algébrique.

Notre résultat principal relatif à l'homologie stable des groupes orthogonaux, démontré au paragraphe 3.2, est le suivant :

ThÉORÈme 1. - Soit $k$ un corps fini de caractéristique impaire. Pour tout foncteur polynomial (voir définition 3.20) $F$ entre k-espaces vectoriels, il existe un isomorphisme naturel :

$$
\operatorname{colim}_{n \in \mathbb{N}} H_{*}\left(O_{n, n}(k) ; F\left(k^{2 n}\right)\right) \simeq \operatorname{Tor}_{*}^{\varepsilon_{k}^{f}}\left(V \mapsto k\left[S^{2}\left(V^{*}\right)\right], F\right)
$$

où $\mathcal{E}_{k}^{f}$ désigne la catégorie des $k$-espaces vectoriels de dimension finie, et $S^{2}$ la seconde puissance symétrique.

Rappelons que les foncteurs $n$-ième puissance tensorielle $T^{n}$ et $n$-ième puissance symétrique $S^{n}$ sont polynomiaux de degré $n$. Par les résultats de [8], la colimite du théorème est donc atteinte pour $n$ fini, en chaque degré homologique. Hormis pour cette considération de stabilisation, le choix des groupes orthogonaux $O_{n, n}$ plutôt que d'autres n'a pas d'importance : toute colimite analogue construite à partir de l'homologie d'autres groupes orthogonaux (associés à des formes quadratiques non dégénérées) sur $k$ est canoniquement isomorphe à celle qu'on considère (cf. remarque 2.24.3).

Le théorème 1 s'obtient par la suite d'isomorphismes suivante :

1 .

$$
\operatorname{colim}_{n \in \mathbb{N}} H_{*}\left(O_{n, n}(k) ; F\left(k^{2 n}\right)\right) \simeq H_{*}\left(\mathcal{E}_{q} ; F\right)
$$

où $\mathcal{E}_{q}$ est la catégorie des $k$-espaces quadratiques non dégénérés de dimension finie. 
Cet isomorphisme vient du cadre général que nous avons évoqué précédemment et de la trivialité de $\operatorname{colim}_{n \in \mathbb{N}} H_{*}\left(O_{n, n}(k) ; k\right)$ (pour $k$ de caractéristique impaire).

2.

$$
H_{*}\left(\mathcal{E}_{q} ; F\right) \simeq H_{*}\left(\mathcal{E}_{q}^{\mathrm{deg}} ; F\right)
$$

où $\mathscr{E}_{q}^{\mathrm{deg}}$ est la catégorie des $k$-espaces quadratiques (éventuellement dégénérés) de dimension finie avec pour morphismes les injections quadratiques. Cette étape constitue le cœur de la démonstration de ce théorème et en est la partie la plus délicate.

3.

$$
H_{*}\left(\mathcal{E}_{q}^{\mathrm{deg}} ; F\right) \simeq \operatorname{Tor}_{*}^{\mathcal{E}_{\text {inj }}^{f}}\left(V \mapsto k\left[S^{2}\left(V^{*}\right)\right], F\right)
$$

où $\mathcal{E}_{\text {inj }}^{f}$ est la sous-catégorie des injections de $\varepsilon_{k}^{f}$. Cet isomorphisme s'obtient par adjonction en observant qu'une forme quadratique sur $V$ est un élément de $S^{2}\left(V^{*}\right)$.

4.

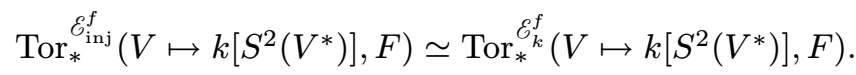

Cet isomorphisme est un cas particulier d'un résultat de Suslin, essentiel pour exprimer l'homologie stable des groupes linéaires en terme d'homologie des foncteurs.

Notons que les isomorphismes 1 et 3 valent pour un foncteur $F$ arbitraire alors que ceux des points 2 et 4 utilisent de manière fondamentale le caractère polynomial de $F$. L'hypothèse de finitude de $k$ n'intervient qu'à l'étape 2.

Revenons plus en détail sur celle-ci. Pour des raisons formelles, il existe une suite spectrale de Grothendieck convergente de la forme :

$$
E_{p, q}^{2}=\operatorname{Tor}_{p}^{\varepsilon_{q}^{\mathrm{deg}}}\left(L_{q}, F\right) \Rightarrow H_{p+q}\left(\varepsilon_{q} ; F\right)
$$

où les foncteurs $L_{q}$ s'obtiennent à partir des foncteurs dérivés d'un adjoint à la précomposition par l'inclusion $\mathcal{E}_{q} \rightarrow \mathcal{E}_{q}^{\mathrm{deg}}$. Les valeurs des foncteurs $L_{q}$ sont données par le $q$-ième groupe d'homologie de sous-groupes explicites du groupe orthogonal. Elles sont l'aboutissement de suites spectrales de Serre dont on peut calculer la deuxième page, mais pas les différentielles. Ces valeurs sont donc inaccessibles pour $q>0$.

Nous contournons ce problème en observant que ces foncteurs $L_{q}$ transforment l'inclusion d'un espace quadratique dans sa somme orthogonale avec un espace non dégénéré, en un isomorphisme. On peut donc les définir depuis la catégorie de fractions où l'on inverse ces inclusions. On montre au théorème 3.17 l'équivalence de cette catégorie avec la catégorie de Burnside sur les espaces vectoriels avec injections. Autrement dit, nos foncteurs $L_{q}$ peuvent être vus comme des foncteurs de Mackey non additifs sur les espaces vectoriels avec injections, ou encore comme des familles de représentations des différents groupes linéaires, par un résultat général d'équivalence de Morita (cf. [33]). Grâce au théorème d'annulation homologique principal de [9], on en déduit un isomorphisme :

$$
E_{p, q}^{2}=\operatorname{Tor}_{p}^{\delta_{q}^{\mathrm{deg}}}\left(L_{q}, F\right) \simeq \operatorname{Tor}_{p}^{\delta_{q}^{\mathrm{deg}}}\left(L_{q}(0), F\right) .
$$

Or $L_{q}(0) \simeq H_{q}\left(O_{\infty}(k) ; k\right)$ est nul pour $q>0$ par le résultat de Fiedorowicz-Priddy cité plus haut. L'isomorphisme 2 en découle.

La conséquence suivante du théorème 1 illustre en quoi la (co)homologie stable est plus régulière que la (co)homologie instable. 
ThÉORÈme 2. - Soient $k$ un corps fini de caractéristique impaire, $F$ et $G$ deux foncteurs polynomiaux entre $k$-espaces vectoriels et $i$, $j$ des entiers. Pour tout entier $n$ assez grand, le produit externe

$$
H^{i}\left(O_{n, n}(k) ; F\left(k^{2 n}\right)\right) \otimes H^{j}\left(O_{n, n}(k) ; G\left(k^{2 n}\right)\right) \rightarrow H^{i+j}\left(O_{n, n}(k) ; F\left(k^{2 n}\right) \otimes G\left(k^{2 n}\right)\right)
$$

est injectif.

Ce résultat est énoncé en terme de cohomologie pour traiter de produits plutôt que de coproduits. Il s'obtient à partir de notre théorème principal par un raisonnement formel dû à Touzé (cf. [30]).

Le théorème 1 permet aussi des calculs explicites, donnés aux théorèmes 4.16 et 4 .17. Nous obtenons entre autres, à l'aide du calcul de [14] des groupes d'extensions entre puissances divisées sur un corps fini, que la cohomologie stable des groupes orthogonaux ou symplectiques à coefficients dans une algèbre polynomiale est elle-même une algèbre de polynômes. Plus précisément :

ThÉORÈme 3. - Soit $k$ un corps fini de cardinal $q$ impair. L'algèbre de cohomologie stable des groupes orthogonaux (resp. symplectiques) sur $k$ à coefficients dans les puissances symétriques est polynomiale sur des générateurs $\alpha_{m, s}$ (resp. $\beta_{m, s}$ ) de bidegré $\left(2 q^{s} m, q^{s}+1\right)$ indexés par des entiers $m \geq 0$ et $s \geq 0$ (resp. $s>0$ ), où le premier degré est le degré homologique.

La partie formelle du présent travail (tout comme l'article [5] sur les groupes symétriques) ne traite que d'homologie stable à coefficients dans un foncteur, pas à coefficients dans un bifoncteur, comme le fait Scorichenko pour les groupes linéaires (à la suite de Betley et Suslin). Néanmoins, contrairement à ce qui advient lorsqu'on étudie l'homologie stable des groupes linéaires (où l'annulation à valeurs dans un foncteur polynomial sans terme constant contraste avec le résultat général pour un bifoncteur polynomial), l'homologie stable des groupes orthogonaux ou symplectiques à coefficients tordus par un bifoncteur ne s'avère pas plus générale que le cas particulier des foncteurs. De fait, toute forme quadratique ou symplectique non dégénérée sur un espace vectoriel $V$ déterminant un isomorphisme entre $V$ et son dual, l'homologie à coefficients tordus par un bifoncteur $B$ (avec une première variable contravariante et la seconde covariante) s'identifie à l'homologie à coefficients tordus par le foncteur $V \mapsto B\left(V^{*}, V\right)$ (cf. remarque 2.13). Comme application, on obtient au corollaire 4.20 l'annulation stable de l'homologie du groupe orthogonal (ou symplectique) sur un corps fini de caractéristique impaire à coefficients dans sa représentation adjointe.

Enfin, signalons que Touzé a tout récemment obtenu (cf. [30]), par des méthodes différentes, des résultats analogues à ceux de cet article pour la cohomologie rationnelle (i.e. comme groupes algébriques) stable des groupes orthogonaux et symplectiques.

Organisation de l'article. - La section 1 présente en détail le cadre général adapté à notre approche de l'homologie stable. On y discute également une classe d'exemples fondamentale, qui contient tous nos cas d'application, et une condition supplémentaire qui intervient dans l'étude de la suite spectrale de la section 2. Celle-ci étudie, dans le formalisme de la section 1, le morphisme puis la suite spectrale naturels qui relient l'homologie d'une catégorie convenable à l'homologie stable de la suite de groupes correspondante. On y discute notamment 
de la simplification de la deuxième page de cette suite spectrale, de son arrêt et de sa comparaison avec les suites spectrales classiques dérivées de constructions du type de la $K$-théorie stable.

La section 3 constitue le cœur de ce travail : elle donne les arguments non formels qui rendront accessible au calcul l'homologie stable des groupes orthogonaux ou symplectiques sur les corps finis, à coefficients tordus raisonnables. Elle identifie d'abord la catégorie de fractions des espaces éventuellement dégénérés où l'inclusion dans la somme orthogonale avec un espace non dégénéré est inversée. Ensuite, elle combine ce résultat avec les théorèmes d'annulation idoines connus en homologie des foncteurs pour obtenir le théorème 1 .

La section 4 donne les applications de ce théorème. On y traite de compatibilité aux (co)produits pour obtenir notamment le théorème 2. On effectue ensuite des calculs d'homologie stable de groupes orthogonaux ou symplectiques. Il faut distinguer la caractéristique impaire, qui se prête à des calculs complets (théorème 3), de la caractéristique 2 où les mêmes méthodes ne suffisent plus, mais où quelques résultats partiels sont déduits des travaux de Troesch ([32]).

Les trois premiers appendices donnent des rappels et des notations sur les catégories de foncteurs utilisés dans le corps de l'article : généralités d'algèbre homologique, foncteurs exponentiels puis quelques équivalences de catégories de foncteurs.

L'appendice D expose les deux résultats sur l'homologie des foncteurs (dus à Djament et Suslin) utilisés dans la démonstration du théorème principal de l'article et en rappelle les arguments.

Dans les deux derniers appendices, on montre comment retrouver rapidement à l'aide de notre formalisme des théorèmes dus à Betley sur l'homologie stable des groupes symétriques et linéaires.

Quelques notations utilisées dans tout l'article. - On se donne un anneau commutatif (unitaire) $\mathbb{k}$ de «base», au-dessus duquel tous les produits tensoriels non spécifiés seront pris. On désigne par $\mathbf{M o d}_{\mathbb{k}}$ la catégorie des $\mathbb{k}$-modules.

Si $\mathscr{C}$ est une catégorie (essentiellement) petite, on note $\mathscr{C}$-Mod la catégorie des foncteurs de $\mathscr{C}$ vers $\operatorname{Mod}_{\mathbb{k}}$. Quelques généralités sur cette catégorie abélienne sont rappelées dans l'appendice A. On pose également Mod- $\mathscr{C}=\mathscr{C}^{\mathrm{op}}$-Mod.

Si $k$ est un corps commutatif, on note $\mathscr{E}(k)$ la catégorie des espaces vectoriels sur $k$ et $\mathcal{E}^{f}(k)$ (ou simplement $\mathcal{E}^{f}$ ) la sous-catégorie pleine des espaces de dimension finie.

La précomposition par un foncteur $Q$ est notée $Q^{*}$.

On note $\mathbb{N}$ l'ensemble des entiers positifs ou nuls.

Remerciements. - Les auteurs témoignent leur gratitude à Vincent Franjou pour ses nombreuses discussions utiles à la réalisation de cet article. Ils remercient Serge Bouc pour leur avoir indiqué l'utilité de l'inversion de Möbius pour obtenir des équivalences de Morita, en particulier le théorème de Pirashvili à la Dold-Kan. Ils sont reconnaissants envers Antoine Touzé pour de fructueuses conversations qui ont permis d'améliorer la présentation ou les résultats de cet article en plusieurs occurrences. Ils le remercient notamment pour leur avoir signalé la remarque 2.13 permettant de traiter le cas des bifoncteurs, ainsi qu'une erreur présente dans une version préliminaire de ce travail, relative à la caractéristique 2 . 
Le second auteur remercie pour son hospitalité le laboratoire de mathématiques Jean Leray (Nantes), où une grande partie de ce travail a été élaboré, ainsi que le programme MATPYL de la Fédération de Recherche 2962 «Mathématiques des Pays de Loire » pour son soutien.

\section{Cadre formel}

Dans cette section, on donne le cadre général de cet article, qui permet de traiter de l'homologie stable des groupes orthogonaux ou symplectiques. On verra, dans les appendices $\mathrm{F}$ et $\mathrm{E}$, que ce cadre s'applique également aux groupes linéaires et symétriques.

\subsection{Hypothèses générales}

On introduit ici des axiomes que l'on supposera vérifiés dans tout l'article. Les exemples et des hypothèses supplémentaires souvent utiles seront donnés dans le paragraphe suivant en effet, tous les cas intéressants d'application de la situation générale, hormis un cas technique apparaissant en cours de démonstration, relèveront desdites hypothèses supplémentaires.

On se donne une catégorie (essentiellement) petite $\mathscr{C}$ et deux foncteurs $S: \mathbb{N} \rightarrow \mathscr{C}$ et $G: \mathbb{N} \rightarrow \mathbf{G r p}$, où $\mathbb{N}$ désigne la catégorie associée à l'ensemble ordonné $\mathbb{N}$ (il y a exactement une flèche d'un entier positif $i$ vers un autre entier positif $j$ si $i \leq j$, et aucune sinon) et Grp la catégorie des groupes.

Avant de donner les trois hypothèses que nous ferons sur $(\mathscr{C}, S, G)$, signalons que l'on pourrait remplacer $\mathbb{N}$ par un autre ensemble ordonné filtrant à droite et possédant un plus petit élément sans modifier la plupart des considérations qui suivent. Cependant, une telle généralisation semble présenter un intérêt modeste dans la mesure où l'on ne connaît aucun exemple qui ne puisse se ramener au cas ici décrit (remplacer l'ensemble ordonné par une partie cofinale à droite contenant le plus petit élément ne modifie guère la situation).

Notre premier axiome est relatif au foncteur $S$, dont il exprime une sorte de propriété de cofinalité :

(C) pour tout objet $c$ de $\mathscr{C}$, il existe $i \in \mathbb{N}$ et un morphisme dans $\mathscr{C}$ de source $c$ et de but $S(i)$.

Notre second axiome renvoie à une forme stable de transitivité de l'action au but des automorphismes sur un ensemble de morphismes :

(W) étant donnés $i \in \mathbb{N}$ et $c \in \mathrm{Ob} \mathscr{C}$, pour tous morphismes $u, v: c \rightarrow S(i)$ de $\mathscr{C}$, il existe $j \geq i$ dans $\mathbb{N}$ et $g \in \operatorname{Aut}_{C} S(j)$ tels que le diagramme

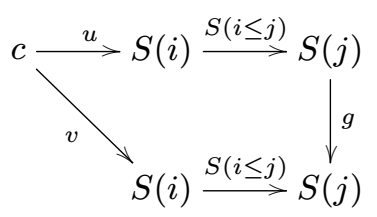

commute.

Dans de nombreux cas, l'axiome suivant, qui implique (W), et exprime une transitivité instable, sera vérifié :

$4^{\mathrm{e}}$ SÉRIE - TOME $43-2010-\mathrm{N}^{\mathrm{o}} 3$ 
$\left(\mathrm{W}^{\prime}\right)$ pour tous $c \in \mathrm{Ob} \mathscr{C}$ et $i \in \mathbb{N}$, le groupe $\operatorname{Aut}_{\mathscr{C}} S(i)$ opère transitivement sur l'ensemble $\operatorname{Hom}_{\mathscr{C}}(c, S(i))$.

L'archétype de résultat fournissant ce type de propriété est le théorème de Witt (cf. paragraphe suivant).

On astreint enfin le foncteur $G$ à satisfaire la propriété de compatibilité au foncteur $S$ suivante :

(G) sur les objets, $G$ est donné par $G(i)=\operatorname{Aut}_{\mathscr{C}}(S(i))$ pour tout $i \in \mathbb{N}$. De plus, on suppose que pour tous entiers $i$ et $j$ tels que $i \leq j$, le morphisme $S(i \leq j): S(i) \rightarrow S(j)$ est $G(i)$-équivariant, où $S(i)$ est muni de l'action à gauche tautologique de $G(i)$ et $S(j)$ de celle déduite du morphisme $G(i \leq j): G(i) \rightarrow G(j)$.

Нy ротнѐSE 1.1. - Dans la suite du paragraphe 1.1, on suppose que $(\mathscr{C}, S, G)$ vérifie les hypothèses $(\mathrm{C}),(\mathrm{W})$ et $(\mathrm{G})$.

Notation 1.2. - La colimite du foncteur $G$ est notée $G_{\infty}$. Pour tout foncteur $F: \mathscr{C} \rightarrow \mathbf{M o d}_{\mathbb{k}}$, on note $F_{\infty}$ la colimite du foncteur composé $\mathbb{N} \stackrel{S}{\rightarrow} \mathscr{C} \stackrel{F}{\rightarrow} \mathbf{M o d}_{\mathbb{k}}$.

Remarque 1.3. - Pour tout foncteur $F: \mathscr{C} \rightarrow \operatorname{Mod}_{\mathbb{k}}$ et tous entiers $i \leq j$, l'application linéaire $(F \circ S)(i \leq j): F(S(i)) \rightarrow F(S(j))$ est $G(i)$-équivariante, par l'axiome (G). Ainsi, $F_{\infty}$ est naturellement un $\mathbb{k}\left[G_{\infty}\right]$-module.

On peut donc donner la définition suivante, qui introduit l'objet d'étude de cet article.

DÉfinition 1.4. - L'homologie stable de la suite de groupes $(G(i))_{i \in \mathbb{N}}$ à coefficients dans $F \in \mathrm{Ob} \mathscr{C}$-Mod est $H_{*}\left(G_{\infty} ; F_{\infty}\right)$.

Remarque 1.5. - Le caractère filtrant à droite de $\mathbb{N}$ implique que le morphisme canonique $\operatorname{colim}_{i \in \mathbb{N}} H_{*}(G(i) ; F(S(i))) \rightarrow H_{*}\left(G_{\infty} ; F_{\infty}\right)$ est un isomorphisme. Dans la suite on identifiera les deux groupes via cet isomorphisme.

On rappelle que l'on note $P_{c}^{\mathscr{C}^{\text {op }}}$ le foncteur $\mathbb{k}\left[\operatorname{Hom}_{\mathscr{C}^{\text {op }}}(c,-)\right]$, appelé projectif standard de Mod- $\mathscr{C}$ (voir appendice $A$ ). On termine ce paragraphe par quelques résultats généraux sur la colimite de ces projectifs standards.

Lemme 1.6. - Le foncteur

$$
P_{\infty}^{\mathscr{C}^{\mathrm{op}}}:=\operatorname{colim}_{i \in \mathbb{N}} P_{S(i)}^{\mathscr{C}^{\mathrm{op}}}
$$

est plat et est muni d'une action du groupe $G_{\infty}$.

De plus, il existe un isomorphisme de $G_{\infty}$-modules

$$
P_{\infty}^{\mathscr{C}^{\mathrm{op}}} \underset{\mathscr{C}}{\otimes} F \simeq F_{\infty}
$$

naturel en $F \in \mathrm{Ob} \mathscr{C}$-Mod.

Démonstration. - La platitude découle du caractère filtrant de $\mathbb{N}$. Comme $G(i)$ agit sur $P_{S(i)}^{\mathscr{C}^{\mathrm{op}}}$, on en déduit également une action canonique de $G_{\infty} \operatorname{sur} P_{\infty}^{\mathscr{C}^{\mathrm{op}}}$.

La dernière partie se vérifie immédiatement. 
LeMme 1.7. - On a

$$
\left(P_{\infty}^{\mathscr{C}^{\mathrm{op}}}\right)_{G_{\infty}} \simeq \mathbb{k}
$$

où $\left(P_{\infty}^{\mathscr{C}^{\mathrm{op}}}\right)_{G_{\infty}}$ désigne les co-invariants de $P_{\infty}^{\mathscr{C}^{\mathrm{op}}}$ par l'action de $G_{\infty}$ et $\mathbb{k}$ est le foncteur constant.

Démonstration. - Il s'agit d'une conséquence directe de l'hypothèse (W).

\subsection{Cas particuliers fondamentaux}

Dans ce paragraphe, on considère une catégorie (essentiellement) petite $\mathscr{C}$ munie d'une structure monoïdale symétrique $\oplus: \mathscr{C} \times \mathscr{C} \rightarrow \mathscr{C}$ dont l'unité sera notée 0 . On fait également l'hypothèse que 0 est objet initial de $\mathscr{C}$.

Quitte à remplacer $\mathscr{C}$ par une catégorie monoïdale symétrique équivalente, on pourra supposer que le foncteur $\oplus$ est strictement associatif et que 0 en est un élément neutre strict, ce qui permet de donner un sens univoque à des expressions comme $A^{\oplus n}$, où $n \in \mathbb{N}$ et $A \in \mathrm{Ob} \mathscr{C}$.

Soit $A \in \mathrm{Ob} \mathscr{C}$. On peut définir un foncteur $S_{A}: \mathbb{N} \rightarrow \mathscr{C}$ par $S_{A}(n)=A^{\oplus n}$ et

$$
S_{A}(n \leq m): A^{\oplus n}=A^{\oplus n} \oplus 0 \stackrel{A^{\oplus n} \oplus\left(0 \rightarrow A^{\oplus(m-n)}\right)}{\longrightarrow} A^{\oplus n} \oplus A^{\oplus(m-n)}=A^{\oplus m} .
$$

Ce choix de fonctorialité consiste, lorsque $\mathscr{C}$ est une catégorie de modules (cf. exemple 1.9.4 ci-dessous), par exemple, à « ajouter des zéros à droite» en termes matriciels.

On définit également un foncteur $G_{A}: \mathbb{N} \rightarrow \operatorname{Grp} \operatorname{par} G_{A}(n)=\operatorname{Aut}_{\mathscr{C}}\left(A^{\oplus n}\right)$ et

$$
G_{A}(n \leq m): \operatorname{Aut}_{\mathscr{C}}\left(A^{\oplus n}\right) \rightarrow \operatorname{Aut}_{\mathscr{C}}\left(A^{\oplus m}\right) \quad u \mapsto u \oplus A^{\oplus(m-n)} .
$$

On vérifie aussitôt le fait suivant :

Proposition 1.8. - Le triplet $\left(\mathscr{C}, S_{A}, G_{A}\right)$ vérifie l'hypothèse $(G)$.

Nous aurons également à considérer l'hypothèse suivante :

(S) pour tout morphisme $f: d \rightarrow c$ de $\mathscr{C}$ et tout $i \in \mathbb{N}$, le morphisme du stabilisateur de $f$ sous l'action de $\operatorname{Aut}_{\mathscr{C}}(c)$ vers le stabilisateur de $S(i) \oplus f$ sous l'action de $\operatorname{Aut}_{\mathscr{C}}(S(i) \oplus c)$ induit par le foncteur $S(i) \oplus$ - est un isomorphisme.

L'hypothèse $(\mathrm{S})$ permettra de donner des renseignements supplémentaires sur la deuxième page de la suite spectrale pour l'homologie de $G_{\infty}$ à coefficients tordus que nous obtiendrons dans la section 2 lorsque les axiomes $(\mathrm{C})$, (W) et $(\mathrm{G})$ sont vérifiés.

Nous introduisons enfin une hypothèse plus forte que (C) mais moins forte que l'essentielle surjectivité du foncteur $S_{A}$ :

$\left(\mathrm{C}^{\prime}\right)$ pour tout objet $c$ de $\mathscr{C}$, il existe un objet $b$ de $\mathscr{C}$ et un entier $i$ tels que $b \oplus c \simeq S(i)$.

Nous terminons ce paragraphe en donnant les exemples fondamentaux qui interviendront dans cet article.

$4{ }^{\mathrm{e}}$ SÉRIE - TOME $43-2010-\mathrm{N}^{\mathrm{o}} 3$ 
Exemple 1.9. - $\quad$ 1. Soient $k$ un corps commutatif (éventuellement de caractéristique 2 ) et $\delta_{q}^{\mathrm{deg}}(k)$ la catégorie (qui sera notée simplement $\mathcal{E}_{q}^{\text {deg }}$ lorsqu'aucune confusion ne peut en résulter) dont les objets sont les espaces quadratiques de dimension finie sur $k$ et les morphismes les applications linéaires injectives compatibles aux formes quadratiques (appelées aussi applications orthogonales). Remarquons qu'une forme quadratique sur un espace vectoriel $V$ est un polynôme homogène de degré 2 sur $V$ ([22]), c'est-à-dire un élément de $S^{2}\left(V^{*}\right)$, où $S^{2}$ est la deuxième puissance symétrique. Comme d'habitude, on note $O(A)$ pour $\operatorname{Aut}_{\mathscr{\delta}_{q}^{\text {deg }}}(A)$ le groupe orthogonal associé à un objet $A$ de $\mathcal{E}_{q}^{\mathrm{deg}}$. On notera par ailleurs $\mathcal{E}_{q}$ la sous-catégorie pleine de $\mathcal{E}_{q}^{\mathrm{deg}}$ dont les objets sont les espaces quadratiques non dégénérés.

La somme orthogonale, notée $\perp$, définit une structure monoïdale symétrique sur $\mathcal{E}_{q}^{\text {deg }}$ dont l'unité 0 est objet initial de $\mathcal{E}_{q}^{\text {deg }}$.

Soit $\mathbf{H}$ l'objet de $\mathcal{E}_{q}$, appelé plan hyperbolique, dont l'espace vectoriel sous-jacent est $k^{2}$ et la forme quadratique l'application $k^{2} \rightarrow k \quad(x, y) \mapsto x y$. Comme d'habitude, on notera $O_{n, n}(k)$ le groupe $G_{\mathbf{H}}(n)$ des automorphismes de $\mathbf{H}^{\perp n}$.

Le triplet ( $\delta_{q}^{\mathrm{deg}}, S_{\mathbf{H}}, G_{\mathbf{H}}$ ) vérifie l'hypothèse $(\mathrm{C})$, car tout espace quadratique se plonge dans un espace quadratique non dégénéré et tout espace quadratique non dégénéré se plonge dans un espace hyperbolique (i.e. un espace quadratique qui est somme directe de sous-espaces totalement isotropes), qui est isomorphe à une somme orthogonale de copies de $\mathbf{H}$ (cf. [26]). Le triplet $\left(\varepsilon_{q}, S_{\mathbf{H}}, G_{\mathbf{H}}\right)$ vérifie pour sa part (C').

Le théorème de Witt montre que l'axiome $\left(\mathrm{W}^{\prime}\right)$ est satisfait, puisque $S_{\mathbf{H}}$ prend ses valeurs dans les espaces non dégénérés.

Le triplet ( $\mathcal{E}_{q}^{\mathrm{deg}}, S_{\mathbf{H}}, G_{\mathbf{H}}$ ) vérifie également l'hypothèse $(\mathrm{S})$ pour la même raison. En effet, si $V$ est un espace quadratique et $H$ un espace non dégénéré, tout automorphisme de $V \perp H$ qui préserve $H$ préserve également son supplémentaire orthogonal, qui n'est autre que $V$ car $H$ est non dégénéré. Par conséquent, tout élément du stabilisateur d'un morphisme $H \perp f: H \perp V \rightarrow H \perp W$ sous l'action de $O(H \perp V)$ stabilise $H \subset H \perp V$ et $W$, donc s'écrit sous la forme $H \perp u$, où $u \in O(W)$ stabilise $f: V \rightarrow W ; u$ est manifestement unique, d'où la satisfaction de (S).

(On pourrait étendre ces considérations à un corps gauche muni d'une involution et aux formes hermitiennes afférentes.)

Remarque 1.10. - On peut remplacer $\mathbf{H}$ par n'importe quel autre $k$-espace quadratique non dégénéré de dimension finie $H$ tel qu'on puisse plonger tout autre $k$-espace quadratique non dégénéré de dimension finie dans une somme de copies de $H$. Si le corps $k$ est fini (auquel cas le treillis des classes d'isomorphisme de $k$-espaces quadratiques non dégénérés de dimension finie est particulièrement simple!), n'importe quel objet non nul $H$ de $E_{q}$ convient.

2. On peut reprendre mutatis mutandis l'exemple précédent en remplaçant les formes quadratiques par les formes bilinéaires alternées. Bornons-nous à préciser nos notations : $\mathcal{E}_{\text {alt }}^{\text {eg }}(k)$ (ou simplement $\mathcal{E}_{\text {alt }}^{\text {deg }}$ ) désignera la catégorie des espaces symplectiques de dimension finie (avec les injections symplectiques pour morphismes) sur le corps 
commutatif $k, \mathcal{E}_{\text {alt }}$ la sous-catégorie pleine des espaces non dégénérés, $\perp$ la somme orthogonale.

On notera aussi $\mathbf{H}$ l'espace symplectique $k^{2}$ muni de la forme $\left((x, y),\left(x^{\prime}, y^{\prime}\right)\right) \mapsto x y^{\prime}-y x^{\prime}$. Le groupe des automorphismes de $A \in \mathrm{Ob} \mathcal{E}_{\text {alt }}^{\text {deg }}$ sera noté $\operatorname{Sp}(A) ; G_{\mathbf{H}}(n)=\operatorname{Sp}\left(\mathbf{H}^{\perp n}\right)$ sera noté $\operatorname{Sp}_{2 n}(k)$.

3. Soit $\Theta$ la catégorie ayant pour objets les ensembles finis et pour morphismes les fonctions injectives. La structure monoïdale symétrique de $\Theta$ est donnée par la réunion disjointe; son unité l'ensemble vide est objet initial de $\Theta$. Soit $A$ un ensemble à un élément fixé. La condition ( $\left.\mathrm{C}^{\prime}\right)$ est vérifiée puisque $S_{A}$ est essentiellement surjectif. On a $G_{A}(n)=\mathfrak{S}_{n}$ (groupe symétrique sur $n$ lettres); on voit facilement que la condition $\left(\mathrm{W}^{\prime}\right)$ est satisfaite. La condition (S) a lieu du fait qu'une bijection d'un ensemble $E$ qui préserve un sous-ensemble $F$ préserve également son complémentaire.

4. Soient $A$ un anneau et $\mathbb{M}(A)$ la catégorie des $A$-modules à gauche projectifs de type fini avec pour morphismes les injections $A$-linéaires scindées, munie de la somme directe, dont l'unité 0 est objet initial. Le triplet $\left(\mathbb{M}(A), S_{A}, G_{A}\right)$ vérifie l'hypothèse $\left(\mathrm{C}^{\prime}\right)$. Comme d'habitude, on note $G L_{n}(A)$ pour $G_{A}(n)$.

L'hypothèse (W) est aussi satisfaite, mais pas (S) si $A$ est non nul et pas non plus $\left(\mathrm{W}^{\prime}\right)$ en général : pour s'en convaincre, on peut considérer un anneau non nul $A$ tel que les $A$-modules à gauche $A$ et $A^{2}$ soient isomorphes, par exemple l'anneau des endomorphismes d'un espace vectoriel de dimension infinie, de sorte que l'injection scindée évidente $A \hookrightarrow A^{2}$, qui n'est pas surjective, ne saurait être conjuguée sous $G L_{2}(A)$ à un isomorphisme $A \stackrel{\simeq}{\rightarrow} A^{2}\left(\left(\mathrm{~W}^{\prime}\right)\right.$ est cependant vraie si $A$ est un anneau assez gentil, par exemple principal). La satisfaction de (W) découle du résultat classique de stabilisation suivant:

Lemme 1.11. - Soient $\mathscr{C}$ une catégorie additive, $M$ et $N$ deux objets de $\mathscr{C}$ et $u: N \rightarrow M$ un monomorphisme scindé de $\mathscr{C}$.

Il existe un automorphisme $g$ de $N \oplus M$ tel que le diagramme

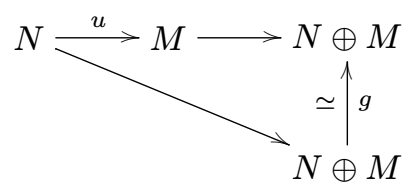

dans lequel les flèches non spécifiées sont les inclusions canoniques, commute.

Démonstration. - Soit $p: M \rightarrow N$ une rétraction de $u$. L'endomorphisme $g$ de $N \oplus M$ donné par la matrice $\left(\begin{array}{cc}0 & p \\ u & \text { Id }\end{array}\right)$ fait commuter le diagramme, et c'est un automorphisme d'inverse donné par $\left(\begin{array}{cc}-\mathrm{Id} & p \\ u & \mathrm{Id}-u p\end{array}\right)$. 


\section{Suite spectrale pour l'homologie stable d'une suite de groupes}

Dans cette section, on introduit une suite spectrale obtenue de manière purement algébrique, convergeant vers l'homologie stable, à coefficients tordus, des familles de groupes qui nous intéressent. Cette suite spectrale est l'ingrédient original de la partie formelle de ce travail ; elle fournit une alternative à la suite spectrale de la $K$-théorie stable considérée dans les travaux de Betley et Scorichenko.

Convention 2.1. - Dans toute cette section, on se donne un triplet $(\mathscr{C}, S, G)$ vérifiant les hypothèses $(C),(W)$ et $(G)$ du paragraphe 1.1.

\subsection{Morphisme de l'homologie stable vers l'homologie de catégorie}

Le but de ce paragraphe est de construire des morphismes naturels :

$$
H_{*}\left(G_{\infty} ; F_{\infty}\right) \rightarrow H_{*}(\mathscr{C} ; F)
$$

où $H_{*}(\mathscr{C} ; F)$ est l'homologie de la catégorie $\mathscr{C}$ à coefficients dans un foncteur $F \in \mathrm{Ob} \mathscr{C}$-Mod, dont on rappelle la définition dans l'appendice A, et

$$
H_{*}\left(G_{\infty} ; F_{\infty}\right) \rightarrow H_{*}\left(\mathscr{C} \times G_{\infty} ; \Pi^{*} F\right)
$$

où l'on voit le groupe $G_{\infty}$ comme une catégorie à un objet et où $\Pi: \mathscr{C} \times G_{\infty} \rightarrow \mathscr{C}$ désigne le foncteur de projection. (On rappelle que $\Pi^{*}: \mathscr{C}$-Mod $\rightarrow \mathscr{C} \times G_{\infty}$-Mod désigne la précomposition par П.)

Ces morphismes naturels peuvent être définis à partir des morphismes d'évaluation. On en donne ici une présentation utilisant une catégorie auxiliaire notée $\widetilde{\mathscr{C}}$, qui interviendra uniquement dans cette section.

L'intérêt de cette catégorie provient de ce qu'on peut obtenir des résolutions plates du foncteur constant $\mathbb{k} \in \mathrm{Ob}$ Mod- $\widetilde{C}$ à partir de résolutions projectives du $G_{\infty}$-module trivial $\mathbb{k}$. Ceci permet, entre autre, d'exprimer $H_{*}\left(G_{\infty} ; F_{\infty}\right)$ en terme d'homologie de la catégorie $\widetilde{\mathscr{C}}$.

Définition 2.2. - Soit $\widetilde{\mathscr{C}}$ la catégorie ayant $\mathbb{N}$ pour ensemble d'objets, telle que $\operatorname{Hom}_{\tilde{C}}(i, j)=G(j)$ pour $i \leq j$ et $\varnothing$ sinon. La composition est définie par $t^{\prime} \circ t:=t^{\prime} \cdot G(j \leq l)(t)$ pour $i \leq j \leq l$ et $t \in \operatorname{Hom}_{\widetilde{\mathscr{C}}}(i, j)=G(j)$ et $t^{\prime} \in \operatorname{Hom}_{\widetilde{\mathscr{C}}}(j, l)=G(l)$.

Notation 2.3. - On désigne par $\widetilde{S}: \mathbb{N} \rightarrow \widetilde{\mathscr{C}}$ le foncteur égal à l'identité sur les objets et associant à chaque relation $i \leq j$ de $\mathbb{N}$ le morphisme de $\widetilde{\mathscr{C}}$ correspondant à $1 \in G(j)$.

Pour la cohérence des notations, on note également $\widetilde{G}:=G: \mathbb{N} \rightarrow \operatorname{Grp}$.

La propriété suivante est immédiate.

Proposition 2.4. - Le triplet $(\widetilde{\mathscr{C}}, \widetilde{S}, \widetilde{G})$ vérifie les hypothèses $(C),\left(W^{\prime}\right)$ et $(G)$.

En revanche, même si $(\mathscr{C}, S, G)$ provient d'une structure monoïdale sur $\mathscr{C}$ comme au paragraphe 1.2, il n'en est pas nécessairement de même pour $(\widetilde{\mathscr{C}}, \widetilde{S}, \widetilde{G})$ (la définition évidente que l'on est tenté de donner à partir de l'addition sur $\mathbb{N}$ et de la structure monoïdale sur $\mathscr{C}$ n'est pas toujours fonctorielle).

Définition 2.5. - On note $Q: \widetilde{\mathscr{C}} \rightarrow \mathscr{C}$ le foncteur donné par $Q(i)=S(i)$ sur les objets et $Q(f)=f \cdot S(i \leq j)$ pour $i \leq j$ dans $\mathbb{N}$ et $f \in \operatorname{Hom}_{\widetilde{\mathscr{C}}}(i, j)=G(j)$ sur les morphismes. 
On vérifie aussitôt la compatibilité de $Q$ à la composition.

Pour $F \in \mathrm{Ob} \mathscr{C}$-Mod, le foncteur $Q^{*}$ fournit un morphisme naturel $H_{*}\left(\widetilde{\mathscr{C}}, Q^{*} F\right) \rightarrow H_{*}(\mathscr{C}, F)$.

Afin d'obtenir le morphisme naturel (1), on identifie dans la suite $H_{*}\left(\widetilde{C} ; Q^{*} F\right)$ et $H_{*}\left(G_{\infty} ; F_{\infty}\right)$. Pour cela nous aurons besoin du résultat suivant qui explique l'avantage de la catégorie $\widetilde{\mathscr{C}} \operatorname{sur} \mathscr{C}$.

LEMme 2.6. - Le foncteur $P_{\infty}^{\widetilde{\mathscr{C}}^{\mathrm{op}}}\left(=\operatorname{colim}_{i \in \mathbb{N}} P_{i}^{\widetilde{\mathscr{C}}^{\mathrm{op}}}\right.$, cf. lemme 1.6) est tel que pour tout $i \in \mathbb{N}$ on a un isomorphisme de $G_{\infty}$-modules :

$$
P_{\infty}^{\widetilde{\mathscr{C}}^{\mathrm{op}}}(i) \simeq \mathbb{k}\left[G_{\infty}\right]
$$

Démonstration. - Pour $i, j \in \mathbb{N}$ tels que $j \leq i$ on a : $P_{i}^{\widetilde{\mathscr{C}}^{\mathrm{op}}}(j)=\mathbb{k}\left[\operatorname{Hom}_{\widetilde{\mathscr{C}}}(j, i)\right] \simeq \mathbb{k}[G(i)]$. Le résultat s'en déduit par passage à la colimite.

REMARQUe 2.7. - Le foncteur $P_{\infty}^{\widetilde{\mathscr{C}}^{\text {op }}}$ n'est pas pour autant un foncteur constant : via l'isomorphisme du lemme précédent, son action sur les morphismes n'est pas donnée par l'identité. En effet, pour $l \leq j \leq i$, si $f \in \operatorname{Hom}_{\tilde{C}^{\circ p}}(j, l)=G(j)$, nous avons ${P_{i}^{\text {op }}}^{\widetilde{\mathscr{C}}^{\mathrm{op}}}(f)([g])=[g \cdot G(j \leq i)(f)]$, pour $g \in G(i)$. Néanmoins nous avons le résultat suivant.

Lemme 2.8. - Le foncteur $\left(P_{\infty}^{\widetilde{\mathscr{C}}^{\mathrm{op}}}\right)_{G_{\infty}}: \widetilde{\mathscr{C}}^{\mathrm{op}} \rightarrow \operatorname{Mod}_{\mathbb{k}}$ est constant en $\mathbb{k}$.

Démonstration. - Notons $\alpha_{j}: G(j) \rightarrow G_{\infty}$, pour $j \in \mathbb{N}$, le morphisme canonique. La remarque précédente montre que, dans l'isomorphisme du lemme 2.6, le morphisme $\mathbb{k}\left[G_{\infty}\right] \simeq P_{\infty}^{\widetilde{\mathscr{C}}^{\text {op }}}(j) \rightarrow P_{\infty}^{\widetilde{\mathscr{C}}^{\text {op }}}(l) \simeq \mathbb{k}\left[G_{\infty}\right]$ induit par un morphisme $l \rightarrow j$ de $\widetilde{\mathscr{C}}$ correspondant à un élément $f$ de $G(j)$ est la multiplication par $\alpha_{j}(f)$. Par passage aux co-invariants il induit donc l'identité de $\mathbb{k}$.

Proposition 2.9. - Il existe un isomorphisme gradué

$$
H_{*}\left(\widetilde{\mathscr{C}} ; Q^{*} F\right) \simeq H_{*}\left(G_{\infty} ; F_{\infty}\right)
$$

naturel en $F \in \mathrm{Ob} \mathscr{C}$-Mod.

Démonstration. - Soit $R_{\bullet} \rightarrow \mathbb{k}$ une résolution projective de $\mathbb{k}$ en tant que $G_{\infty}$-module. Par le lemme 2.6 le foncteur $-\underset{G_{\infty}}{\otimes} P_{\infty}^{\widetilde{\mathscr{C}}^{\mathrm{op}}}: \operatorname{Mod}_{\mathbb{k}\left[G_{\infty}\right]} \rightarrow \operatorname{Mod}-\widetilde{\mathscr{C}}$ est exact. On en déduit un complexe exact $R_{\bullet} \otimes_{G_{\infty}} P_{\infty}^{\widetilde{\mathscr{C}}^{\mathrm{op}}} \rightarrow \mathbb{k} \otimes_{G_{\infty}} P_{\infty}^{\widetilde{\mathscr{C}}^{\mathrm{op}}}$ dans Mod- $\widetilde{\mathscr{C}}$. Or $\mathbb{k} \otimes_{G_{\infty}} P_{\infty}^{\widetilde{C}^{\mathrm{op}}} \simeq\left({\widetilde{\mathscr{C}}_{\infty}^{\mathrm{op}}}\right)_{G_{\infty}} \simeq \mathbb{k}$ d'après le lemme 2.8. De plus les foncteurs $R_{i} \otimes_{G_{\infty}} P_{\infty}^{\widetilde{\mathscr{C}}^{\mathrm{op}}}$ de Mod- $\widetilde{C}$ sont plats comme $P_{\widetilde{C}_{\text {op }}}^{\widetilde{\mathscr{O}}_{\mathrm{op}}}$ puisque les $G_{\infty}$-modules $R_{i}$ sont projectifs. On en déduit que $R_{\bullet} \otimes_{G_{\infty}} P_{\infty}^{\widetilde{\mathscr{C}}^{\text {op }}} \rightarrow \mathbb{k} \otimes_{G_{\infty}} P_{\infty}^{\widetilde{\mathscr{C}}^{\text {op }}} \simeq \mathbb{k}$ est une résolution plate de $\mathbb{k} \in$ Ob Mod- $\widetilde{C}$.

On utilise maintenant l'isomorphisme canonique

$$
\left(M \underset{G_{\infty}}{\otimes} P_{\infty}^{\widetilde{\mathscr{C}}^{\mathrm{op}}}\right) \underset{\widetilde{\mathscr{C}}}{\otimes} Q^{*} F \simeq M \underset{G_{\infty}}{\otimes}\left(P_{\infty}^{\widetilde{\mathscr{C}}^{\mathrm{op}}} \underset{\widetilde{\mathscr{C}}}{\otimes} Q^{*} F\right)
$$


naturel en $F$ et en le $G_{\infty}$-module $M$, associé aux isomorphismes naturels de $G_{\infty}$-modules

$$
P_{\infty}^{\widetilde{\mathscr{C}}^{\mathrm{op}}} \underset{\widetilde{\mathscr{C}}}{\otimes} Q^{*} F \simeq\left(Q^{*} F\right)_{\infty} \simeq F_{\infty}
$$

dont le premier est déduit du lemme 1.6 et de la proposition 2.4 et le second s'obtient par inspection.

On peut donc définir le morphisme canonique (1) par la composition

$$
H_{*}\left(G_{\infty} ; F_{\infty}\right) \stackrel{\simeq}{\longrightarrow} H_{*}\left(\widetilde{\mathscr{C}} ; Q^{*} F\right) \rightarrow H_{*}(\mathscr{C} ; F)
$$

de l'isomorphisme de la proposition précédente et du morphisme induit par $Q$. On peut vérifier aisément que ce morphisme est toujours un isomorphisme en degré 0 , ce qui découlera des résultats du paragraphe suivant, qui permettent d'étudier son comportement en tout degré.

Remarque 2.10. - Pour formel et élémentaire qu'il soit, ce résultat en degré 0 peut déjà procurer un point de vue efficace sur des calculs de co-invariants stables. Par exemple (anticipant sur les résultats que nous donnerons par la suite en tout degré homologique, résultats qui sont eux non formels et considérablement plus difficiles qu'en degré 0 ) si $\mathbb{k}=k$ est un corps fini, on peut en déduire sans trop de peine le fait que

$$
\operatorname{colim}_{n \in \mathbb{N}} H_{0}\left(O_{n, n}(k) ; \Gamma^{*}\left(k^{2 n}\right)\right) \quad\left(\text { resp. colim } H_{n \in \mathbb{N}} H_{0}\left(\operatorname{Sp}_{2 n}(k) ; \Gamma^{*}\left(k^{2 n}\right)\right)\right)
$$

est isomorphe à l'espace vectoriel des transformations naturelles de $\Gamma^{*}$ (foncteur (gradué) puissance divisée sur les $k$-espaces vectoriels) vers le foncteur $V \mapsto k^{S^{2}\left(V^{*}\right)}$ (resp. $\left.V \mapsto k^{\Lambda^{2}\left(V^{*}\right)}\right)$, où l'étoile indique cette fois la dualité.

L'article de Kuhn [20] (cf. son théorème 1.6) montre comment calculer ces espaces vectoriels gradués, à l'aide du lien fondamental établi entre les foncteurs entre $k$-espaces vectoriels et algèbre de Steenrod sur $k$ (au moins lorsque le corps fini $k$ est premier), établi par Henn, Lannes et Schwartz (on pourra consulter le premier article de Schwartz dans l'ouvrage [13] à ce sujet). Ce résultat est déjà remarquable car le calcul direct des co-invariants (3) n'est pas du tout immédiat!

Pour définir le morphisme (2), nous utiliserons le foncteur donné par la proposition immédiate suivante :

Proposition 2.11. - Il existe un foncteur $J: \widetilde{\mathscr{C}} \rightarrow G_{\infty}$ envoyant chaque flèche $u \in \operatorname{Hom}_{\tilde{C}}(i, j)=G(j)$ sur son image canonique dans $G_{\infty}$.

Le morphisme naturel (2) est défini par la composition

$$
H_{*}\left(G_{\infty} ; F_{\infty}\right) \stackrel{\simeq}{\rightarrow} H_{*}\left(\widetilde{\mathscr{C}} ; Q^{*} F\right)=H_{*}\left(\widetilde{\mathscr{C}} ;(Q, J)^{*}\left(\Pi^{*} F\right)\right) \rightarrow H_{*}\left(\mathscr{C} \times G_{\infty} ; \Pi^{*} F\right)
$$

(on rappelle que $\Pi$ désigne la projection $\mathscr{C} \times G_{\infty} \rightarrow \mathscr{C}$ ) composée de l'isomorphisme de la proposition 2.9 et du morphisme induit $\operatorname{par}(Q, J)$.

On notera que le morphisme (1) n'est autre que la composée du morphisme (2) avec le morphisme naturel $H_{*}\left(\mathscr{C} \times G_{\infty} ; \Pi^{*} F\right) \rightarrow H_{*}(\mathscr{C} ; F)$ induit par $\Pi$.

La proposition 2.9 admet la généralisation suivante : 
Proposition 2.12. - Il existe un isomorphisme gradué

$$
H_{*}\left(\widetilde{C} ;(Q, J)^{*} X\right) \simeq H_{*}\left(G_{\infty} ; X_{\infty}\right)
$$

naturel en $X \in \mathrm{Ob}\left(\mathscr{C} \times G_{\infty}\right)$-Mod, où $X_{\infty}=\operatorname{colim}_{n \in \mathbb{N}} X(S(n))$ est muni de l'action diagonale de $G_{\infty}$ (cet espace vectoriel est naturellement muni d'une action de $G_{\infty} \times G_{\infty}$, dont un facteur agit comme dans la remarque 1.3 et l'autre par l'action tautologique de $G_{\infty}$ sur $\left.\mathscr{C} \times G_{\infty}\right)$.

Démonstration. - Elle est complètement analogue à celle de la proposition 2.9, en remplaçant $Q^{*} F$ par $(Q, J)^{*} X$.

Remarque 2.13. - Pour donner une généralisation des considérations précédentes en termes de bifoncteurs, on a besoin de données supplémentaires. Par exemple, si $B$ est un bifoncteur sur la catégorie $\mathbb{P}(A)$ des $A$-modules à gauche projectifs de type fini sur un anneau $A$, i.e. un objet de $\mathbb{P}(A)^{\mathrm{op}} \times \mathbb{P}(A)$-Mod, on définit l'homologie stable des groupes linéaires sur $A$ à coefficients dans $B$ comme étant $H_{*}\left(G L_{\infty}(A) ; B_{\infty}\right)$, où $B_{\infty}$ est la colimite des $B\left(A^{n}, A^{n}\right)$ construite à partir des projections $A^{n+1} \rightarrow A^{n}$ sur les $n$ premiers facteurs (pour la première variable, contravariante) et des inclusions $A^{n} \hookrightarrow A^{n+1}$ des $n$ premiers facteurs (pour la seconde variable, covariante).

Néanmoins, dans le cas des groupes orthogonaux ou symplectiques, on n'obtient rien de plus général par une telle procédure. En effet, toute forme quadratique non dégénérée sur un espace vectoriel déterminant un isomorphisme de celui-ci sur son dual, on dispose, à côté du foncteur d'oubli de la forme quadratique $\mathcal{E}_{q} \rightarrow \mathcal{E}^{f}$, d'un foncteur $\mathcal{E}_{q} \rightarrow\left(\mathcal{E}^{f}\right)^{\text {op }}$ égal à l'identité sur les objets, de sorte que l'homologie stable des groupes orthogonaux à coefficients dans un bifoncteur sur $\mathcal{E}^{f}$ (les bifoncteurs définis déjà sur les espaces vectoriels s'avèrent les plus intéressants) n'est autre que l'homologie stable du foncteur obtenu en précomposant avec :

$$
\mathcal{E}_{q} \rightarrow\left(\mathcal{E}^{f}\right)^{\mathrm{op}} \times \mathcal{E}^{f}
$$

On peut procéder de manière analogue pour les formes symplectiques.

\subsection{Suite spectrale fondamentale}

Soit $(Q, J) !:$ Mod- $\widetilde{\mathscr{C}} \rightarrow \operatorname{Mod}-\left(\mathscr{C} \times G_{\infty}\right)$ le foncteur défini (à isomorphisme canonique près) par l'isomorphisme naturel

$$
\forall X \in \mathrm{Ob}\left(\mathscr{C} \times G_{\infty}\right) \text {-Mod } \forall Y \in \text { Ob Mod- } \widetilde{\mathscr{C}} \quad Y \underset{\mathscr{C}}{\otimes}(Q, J)^{*}(X) \simeq(Q, J)_{!}(Y) \underset{\mathscr{C} \times G_{\infty}}{\otimes} X
$$

(cf. proposition A.2).

Cet isomorphisme se dérive en une suite spectrale de Grothendieck (homologique) de terme $E^{2}$ donné par

$$
E_{p, q}^{2}=\operatorname{Tor}_{p}^{\mathscr{C} \times G_{\infty}}\left(\mathbb{L}_{q}(Q, J)_{!}(Y), X\right)
$$

et d'aboutissement $\operatorname{Tor}_{*}^{\widetilde{\mathscr{C}}}\left(Y,(Q, J)^{*}(X)\right)$. Les foncteurs $\mathbb{L}_{q}(Q, J)_{!}(Y)$ désignent les dérivés à gauche du foncteur exact à droite $(Q, J)$ ! ; cette suite spectrale est naturelle en $X$ et concentrée dans le premier quadrant, donc en particulier convergente.

On s'intéresse maintenant au cas où $Y$ est le foncteur constant $\mathbb{k}$. 
Proposition 2.14. - Pour tout entier $q>0$, on a $\mathbb{L}_{q}(Q, J)_{!}(\mathbb{k})=0$.

De plus, $(Q, J)_{!}(\mathbb{k})$ est donné par un isomorphisme naturel $(Q, J)_{!}(\mathbb{k})(c) \simeq\left(P_{c}^{\mathscr{C}}\right)_{\infty}$ pour $c \in \mathrm{Ob} \mathscr{C}$.

Démonstration. - La formule (12) (appendice A, proposition A.2) et la proposition 2.12 procurent des isomorphismes

$$
\mathbb{L}_{q}(Q, J)_{!}(\mathbb{k})(c)=H_{q}\left(\widetilde{\mathscr{C}} ;(Q, J)^{*}\left(P_{c}^{\mathscr{C} \times G_{\infty}}\right)\right) \simeq H_{q}\left(G_{\infty} ;\left(P_{c}^{\mathscr{C} \times G_{\infty}}\right)_{\infty}\right) .
$$

Comme $\left(P_{c}^{\mathscr{C} \times G_{\infty}}\right)_{\infty} \simeq\left(P_{c}^{\mathscr{C}}\right)_{\infty} \otimes \mathbb{k}\left[G_{\infty}\right]$ comme $G_{\infty}$-modules, cela démontre la proposition.

On déduit donc de notre suite spectrale et de la proposition 2.12:

Corollaire 2.15. - Il existe un isomorphisme gradué naturel

$$
H_{*}\left(G_{\infty} ; X_{\infty}\right) \simeq \operatorname{Tor}_{*}^{\mathscr{C} \times G_{\infty}}((Q, J) !(\mathbb{k}), X)
$$

pour $X \in \mathrm{Ob}\left(\mathscr{C} \times G_{\infty}\right)$-Mod.

En particulier, il existe un isomorphisme naturel

$$
H_{*}\left(G_{\infty} ; F_{\infty}\right) \simeq \operatorname{Tor}_{*}^{\mathscr{C} \times G_{\infty}}\left((Q, J) !(\mathbb{k}), \Pi^{*} F\right)
$$

pour $F \in \mathrm{Ob} \mathscr{C}$-Mod.

Pour étudier plus avant ces groupes, nous aurons besoin du résultat classique suivant sur l'homologie d'un produit de deux catégories:

Proposition 2.16. - Soient $\mathscr{G}$ et $\mathcal{B}$ deux petites catégories et $\Pi: \mathscr{G} \times \mathscr{B} \rightarrow \mathscr{G}$ le foncteur de projection.

Il existe une suite spectrale (du premier quadrant)

$$
E_{p, q}^{2}=\operatorname{Tor}_{p}^{A}\left(A \mapsto H_{q}(\mathcal{B} ; Y(A,-)), F\right) \Rightarrow \operatorname{Tor}_{p+q}^{\mathscr{Q} \times \mathscr{B}}\left(Y, \Pi^{*} F\right)
$$

fonctorielle en $F \in \mathrm{Ob} \mathscr{G}$-Mod et $Y \in \mathrm{Ob} \operatorname{Mod}-(\mathscr{G} \times \mathcal{B})$.

En particulier, il existe deux suites spectrales de Künneth

$$
\begin{aligned}
\mathrm{I}_{p, q}^{2} & =H_{p}\left(\mathscr{G} ; A \mapsto H_{q}(\mathscr{B} ; X(A,-))\right) \Rightarrow H_{p+q}(\mathscr{C} \times \mathscr{B} ; X), \\
\mathrm{II}_{p, q}^{2} & =H_{p}\left(\mathscr{B} ; B \mapsto H_{q}(\mathscr{C} ; X(-, B))\right) \Rightarrow H_{p+q}(\mathscr{C} \times \mathscr{B} ; X)
\end{aligned}
$$

fonctorielles en $X \in \mathrm{Ob}(\mathscr{G} \times \mathcal{B})$-Mod.

Démonstration. - Il existe un isomorphisme naturel

$$
Y \underset{\mathscr{Q} \times \mathscr{B}}{\otimes} \Pi^{*}(F) \simeq\left(A \mapsto H_{0}(\mathscr{B} ; Y(A,-))\right) \underset{\mathscr{Q}}{\otimes} F
$$

La suite spectrale recherchée est la suite spectrale de Grothendieck correspondante.

Afin d'examiner la forme que prend cette suite spectrale dans le cas qui nous intéresse, nous introduisons les définitions suivantes. 
Notation 2.17. - Soient $i$ et $j$ deux éléments de $\mathbb{N}$ tels que $i \leq j$. Nous désignerons par $\operatorname{St}_{\mathscr{C}}(i, j)$ le stabilisateur de $S(i \leq j) \in \operatorname{Hom}_{\mathscr{C}}(S(i), S(j))$ sous l'action à gauche canonique de $G(j)$.

Nous noterons $\operatorname{St}_{\mathscr{C}}(i)$ la colimite $\operatorname{sur} j \geq i$ des groupes $\operatorname{St}_{\mathscr{C}}(i, j)$. Nous identifierons $\operatorname{St}_{\mathscr{C}}(i)$ avec son image dans le groupe $G_{\infty}$.

On remarque que l'on a $\operatorname{St}_{\mathscr{C}}(j) \subset \operatorname{St}_{\mathscr{C}}(i)$ pour $i \leq j$. Cela permet de considérer $\mathrm{St}_{\mathscr{C}}$ comme un foncteur de but Grp et de source $\mathbb{N}^{\text {op }}$.

Nous aurons besoin, dans les cas où le foncteur $S$ n'est pas essentiellement surjectif, de la généralisation suivante de ces stabilisateurs, qui s'effectue au prix d'une perte de fonctorialité.

Notation 2.18. - Choisissons, conformément à l'axiome (C), un morphisme $c \stackrel{u_{c}}{\longrightarrow} S\left(i_{c}\right)$ de $\mathscr{C}$ pour tout objet $c$ de $\mathscr{C}$. Pour tout entier naturel $j \geq i_{c}$, on note $\operatorname{St}(c, j)$ le stabilisateur de $c \stackrel{u_{c}}{\longrightarrow} S\left(i_{c}\right) \stackrel{S\left(i_{c} \leq j\right)}{\longrightarrow} S(j)$ sous l'action à gauche canonique de $G(j)$.

Nous noterons $\operatorname{St}(c)$ la colimite sur $j \geq i_{c}$ des groupes $\operatorname{St}(c, j)$. Nous identifierons $\operatorname{St}(c)$ avec son image dans le groupe $G_{\infty}$.

L'hypothèse (W) montre que changer le choix des $i_{c}$ et des $u_{c}$ ne modifie pas, à conjugaison près, les groupes $\operatorname{St}(c)$ obtenus. Pour la même raison, tout morphisme $f: b \rightarrow c$ de $\mathscr{C}$ fait de $\operatorname{St}(c)$ un sous-groupe d'un conjugué de $\operatorname{St}(b)$ dans $G_{\infty}$.

Malgré le manque de fonctorialité sur les groupes $\operatorname{St}(c)$, la remarque précédente et la trivialité en homologie de l'action des automorphismes intérieurs d'un groupe font de $c \mapsto H_{*}(\operatorname{St}(c) ; \mathbb{k})$ un foncteur de $\mathscr{C}^{\mathrm{op}}$ vers les $\mathbb{k}$-modules gradués.

Proposition 2.19. - Il existe une suite spectrale

$$
E_{p, q}^{2}=\operatorname{Tor}_{p}^{\mathscr{C}}\left(c \mapsto H_{q}(\operatorname{St}(c) ; \mathbb{k}), F\right) \Rightarrow H_{p+q}\left(G_{\infty} ; F_{\infty}\right)
$$

naturelle en $F \in \mathrm{Ob} \mathscr{C}$-Mod.

Démonstration. - Le $G_{\infty}$-module $\left(P_{c}^{\mathscr{C}}\right)_{\infty}$ s'identifie à $\mathbb{k}\left[G_{\infty} / \operatorname{St}(c)\right]$ grâce à l'axiome $(\mathrm{W})$. La conclusion découle donc des propositions 2.14 et 2.16 et du lemme de Shapiro.

Remarque 2.20. - $\quad$ 1. En utilisant la proposition 2.9 plutôt que la proposition 2.12, on obtient une suite spectrale naturelle

$$
E_{p, q}^{2}=\operatorname{Tor}_{p}^{\mathscr{C}}\left(\mathbb{L}_{*} Q_{!}(\mathbb{k}), F\right) \Rightarrow H_{p+q}\left(G_{\infty} ; F_{\infty}\right) .
$$

On vérifie aisément qu'existent des isomorphismes naturels $\mathbb{L}_{q} Q_{!}(\mathbb{k})(c) \simeq H_{q}(\operatorname{St}(c) ; \mathbb{k})$ et que la suite spectrale précédente est isomorphe à celle de la proposition 2.19.

L'intérêt de la présentation qu'on donne réside dans la possibilité d'utiliser des arguments généraux d'effondrement pour les suites spectrales de la proposition 2.16 comme on le verra dans le paragraphe suivant.

2. Les suites spectrales de ce paragraphe possèdent également des propriétés de fonctorialité relativement à $\mathscr{C}$ qu'on laisse au lecteur le soin d'énoncer.

Notation 2.21. - Lorsqu'aucune ambiguïté n'est possible $\operatorname{sur}(\mathscr{C}, S, G)$ ou $\mathbb{k}$, on notera $L_{q}$ le foncteur $c \mapsto H_{q}(\mathrm{St}(c) ; \mathbb{k})$.

C'est dans la proposition suivante que l'intérêt de l'hypothèse (S) apparaît. 
Proposition 2.22. - Supposons que les foncteurs $S$ et $G$ proviennent d'une structure monoïdale symétrique sur $\mathscr{C}$ comme au paragraphe 1.2 et que l'hypothèse $(S)$ est satisfaite.

Alors pour tous entiers $n$, $i$ et tout objet $c$ de $\mathscr{C}$, le foncteur $L_{n}$ transforme le morphisme canonique $c \rightarrow S(i) \oplus$ c en un isomorphisme.

Si de plus $S$ vérifie l'hypothèse $\left(C^{\prime}\right)$, alors le foncteur $L_{n}$ est constant en $H_{n}\left(G_{\infty} ; \mathbb{k}\right)$. La suite spectrale de la proposition 2.19 prend donc naturellement la forme

$$
E_{p, q}^{2} \simeq \operatorname{Tor}_{p}^{\mathscr{C}}\left(H_{q}\left(G_{\infty} ; \mathbb{k}\right), F\right) \Rightarrow H_{p+q}\left(G_{\infty} ; F_{\infty}\right) .
$$

Démonstration. - On peut supposer que $i_{S(i) \oplus c}=S(i) \oplus i_{c}$ et $u_{S(i) \oplus c}=S(i) \oplus u_{c}$ (cf. supra). Pour tout entier $j \geq i_{c}$, le foncteur $S(i) \oplus-$ induit donc un isomorphisme $\operatorname{St}(c, j) \stackrel{\simeq}{\longrightarrow} \operatorname{St}(S(i) \oplus c, i+j)$, par l'axiome (S).

Identifiant ces deux groupes via cet isomorphisme, on voit que la flèche induite par le morphisme canonique $c \rightarrow S(i) \oplus c$ s'identifie au morphisme de groupes $\operatorname{St}(c, j) \hookrightarrow \operatorname{St}(c, i+$ j) induit par

$$
G(j)=\operatorname{Aut}_{\mathscr{C}}\left(A^{\oplus j}\right) \rightarrow G(i+j)=\operatorname{Aut}_{\mathscr{C}}\left(A^{\oplus(i+j)}\right) \quad u \mapsto A^{\oplus i} \oplus u .
$$

Celui-ci est conjugué au morphisme $u \mapsto u \oplus A^{\oplus i}$ qui intervient dans la colimite définissant $G_{\infty}$ par l'automorphisme de $A^{\oplus(i+j)}$

$$
A^{\oplus(i+j)}=A^{\oplus i} \oplus A^{\oplus j} \simeq A^{\oplus j} \oplus A^{\oplus i}=A^{\oplus(i+j)}
$$

donné par l'échange des facteurs.

Comme les automorphismes intérieurs n'agissent pas en homologie, cela fournit la première partie du résultat, grâce à la proposition 2.19.

Supposons maintenant que $S$ vérifie l'hypothèse $\left(\mathrm{C}^{\prime}\right)$. Soient $b$ et $c$ des objets de $\mathscr{C}$ tels qu'existent $i \in \mathbb{N}$ et un isomorphisme $b \oplus c \simeq S(i)$. Ce qui précède montre que, pour tout $a \in \mathrm{Ob} \mathscr{C}$ et tout $n \in \mathbb{N}$, l'application linéaire $L_{n}(a \rightarrow a \oplus c)$ a un inverse à droite, donné à isomorphisme près par $L_{n}(a \oplus c \rightarrow a \oplus c \oplus b)$ (qui a donc lui un inverse à gauche!); pour la même raison, ce dernier morphisme a un inverse à droite. Donc $L_{n}(a \oplus c \rightarrow a \oplus c \oplus b)$ puis $L_{n}(a \rightarrow a \oplus c)$ sont des isomorphismes.

Considérons à présent un morphisme $f: d \rightarrow c$ quelconque de $\mathscr{C}$ et montrons que c'est un $L_{*}$-isomorphisme. On choisit des objets $a$ et $b$ de $\mathscr{C}$ et des entiers $i$ et $j$ tels qu'existent des isomorphismes $a \oplus d \simeq S(j)$ et $b \oplus c \simeq S(i)$. On peut ensuite trouver, par l'axiome (W), un entier $l$, qu'on peut supposer supérieur à $i$ et $j$, et un automorphisme $g$ de $S(l)$ tels que le diagramme suivant commute :

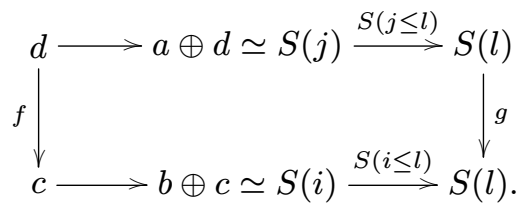

Toutes les flèches horizontales sont des $L_{*}$-isomorphismes par ce qui précède, de même que $g$ qui est un isomorphisme, donc c'est aussi le cas de $f$, ce qui achève la démonstration.

Remarque 2.23. - C'est dans cette démonstration qu'apparaît l'intérêt de supposer symétrique la structure monoïdale de $\mathscr{C}$, hypothèse qui n'intervient nulle part ailleurs dans les constructions ou démonstrations. 
Remarque 2.24. - $\quad$ 1. Dans le cas de la catégorie $\mathbb{M}(A)$ des modules à gauche projectifs de type fini sur un anneau $A$ avec injections scindées (cf. exemple 1.9.4), l'hypothèse $\left(\mathrm{C}^{\prime}\right)$ est satisfaite mais pas $(\mathrm{S})$; néanmoins, la conclusion de la proposition précédente a encore lieu. Cela provient de résultats établis par Betley dans [3]. Nous en donnons une démonstration, fondée sur les travaux de Scorichenko, dans l'appendice F.

2. Toutes les hypothèses de la proposition sont satisfaites par la catégorie $\Theta$ de l'exemple 1.9.3. Cela permet de retrouver les résultats de Betley (cf. [5]) sur l'homologie stable des groupes symétriques. C'est ce que nous ferons dans l'appendice E.

3. Toutes les hypothèses de la proposition 2.22 sont également satisfaites dans les catégories $\mathcal{E}_{q}(k)$ et $\mathscr{E}_{\text {alt }}(k)$ (où $k$ est un corps commutatif) munies de la somme orthogonale et de l'objet $\mathbf{H}$ (cf. exemples 1.9. 1 et 1.9.2). Cependant, les résultats de ce corollaire sont peu maniables dans ce cas, ce qui nous amènera à travailler plutôt dans les catégories $\mathcal{E}_{q}^{\mathrm{deg}}$ et $\mathcal{E}_{\text {alt }}^{\mathrm{deg}}$, dans les sections suivantes.

On remarquera déjà que, le choix de l'espace quadratique $\mathbf{H}$ n'ayant pas réellement d'importance (cf. remarque 1.10), l'homologie de la catégorie $\mathcal{E}_{q}$ calcule l'homologie stable de tous les groupes orthogonaux sur $k:$ le morphisme canonique de la colimite des homologies des groupes orthogonaux $O_{n, n}$ (qui correspondent au choix de $\mathbf{H}$ ) vers la colimite des homologies de tous les groupes orthogonaux (associés à des formes quadratiques non dégénérées) est un isomorphisme. (On peut le voir directement très rapidement : c'est une simple conséquence de l'inaction des automorphismes intérieurs en homologie, comme dans la démonstration de la proposition 2.22.)

\subsection{Arrêt de la suite spectrale et comparaison à la $K$-théorie stable}

Nous commençons par donner une condition suffisante pour que le morphisme $H_{*}\left(G_{\infty} ; F_{\infty}\right) \rightarrow H_{*}\left(\mathscr{C} \times G_{\infty} ; \Pi^{*} F\right)$ du paragraphe 2.1 soit un isomorphisme.

Proposition 2.25. - Supposons que la catégorie C possède un objet initial, noté 0 , de sorte qu'il existe, pour tout objet $G$ de Mod- $\mathscr{C}$, un morphisme naturel de $G$ vers le foncteur $G(0)$.

Soit $F \in \mathrm{Ob} \mathscr{C}$-Mod tel que le morphisme $L_{q} \rightarrow L_{q}(0)$ induise un isomorphisme $\operatorname{Tor}_{*}^{\mathscr{C}}\left(L_{q}, F\right) \stackrel{\simeq}{\longrightarrow} \operatorname{Tor}_{*}^{\mathscr{C}}\left(L_{q}(0), F\right)$ en homologie pour tout $q \in \mathbb{N}$.

Alors le morphisme (2) : $H_{*}\left(G_{\infty} ; F_{\infty}\right) \rightarrow H_{*}\left(\mathscr{C} \times G_{\infty} ; \Pi^{*} F\right)$ est un isomorphisme.

Démonstration. - Par la proposition 2.16 et le corollaire 2.15,

$$
H_{*}\left(G_{\infty} ; F_{\infty}\right) \simeq \operatorname{Tor}_{*}^{\mathscr{C} \times G_{\infty}}\left(c \mapsto\left(P_{c}^{\mathscr{C}}\right)_{\infty}, \Pi^{*} F\right) \text { et } H_{*}\left(\mathscr{C} \times G_{\infty} ; \Pi^{*} F\right) \simeq \operatorname{Tor}_{*}^{\mathscr{C} \times G_{\infty}}\left(\mathbb{k}, \Pi^{*} F\right)
$$

sont respectivement l'aboutissement de suites spectrales convergentes de deuxièmes pages :

$$
\mathrm{I}_{p, q}^{2}=\operatorname{Tor}_{p}^{\mathscr{C}}\left(L_{q}, F\right) \quad \text { et } \quad \mathrm{II}_{p, q}^{2}=\operatorname{Tor}_{p}^{\mathscr{C}}\left(L_{q}(0), F\right)
$$

(puisque $\left.L_{q}(0) \simeq H_{q}\left(G_{\infty} ; \mathbb{k}\right)\right)$. Le morphisme $\left(c \mapsto\left(P_{c}^{\mathscr{C}}\right)_{\infty}\right) \rightarrow\left(P_{0}^{\mathscr{C}}\right)_{\infty} \simeq \mathbb{k}$ induit par hypothèse un isomorphisme $\mathrm{I}_{p, q}^{2} \rightarrow \mathrm{II}_{p, q}^{2}$, il induit donc un isomorphisme entre les aboutissements des suites spectrales, d'où la proposition.

$4^{\mathrm{e}}$ SÉRIE - TOME $43-2010-\mathrm{N}^{\mathrm{o}} 3$ 
À partir de ce résultat, nous donnons un critère simple pour que la suite spectrale qui nous intéresse s'effondre à la deuxième page; on obtient même mieux : non seulement le terme $E^{\infty}$, mais aussi l'aboutissement de la suite spectrale (qui en diffère par une filtration), sont isomorphes au terme $E^{2}$.

Proposition 2.26. - Faisons les trois hypothèses suivantes:

1. l'anneau $\mathbb{k}$ est de dimension homologique au plus 1 ;

2. la catégorie $\mathscr{C}$ possède un objet initial 0 ;

3. $F \in \mathrm{Ob} \mathscr{C}$-Mod est tel que, pour tout entier q, le morphisme $L_{q} \rightarrow L_{q}(0)$ induise un isomorphisme $\operatorname{Tor}_{*}^{\mathscr{C}}\left(L_{q}, F\right) \stackrel{\simeq}{\longrightarrow} \operatorname{Tor}_{*}^{\mathscr{C}}\left(L_{q}(0), F\right)$ en homologie.

Alors il existe des isomorphismes

$$
H_{n}\left(G_{\infty} ; F_{\infty}\right) \simeq H_{n}\left(\mathscr{C} \times G_{\infty} ; \Pi^{*} F\right) \simeq \bigoplus_{p+q=n} \operatorname{Tor}_{p}^{\mathscr{C}}\left(H_{q}\left(G_{\infty} ; \mathbb{k}\right), F\right)
$$

naturels en $F$.

Les cas les plus importants sont ceux où $\mathbb{k}$ est un corps ou égale $\mathbb{Z}$. On remarque que sous les hypothèses de la proposition 2.22 les conditions 2 et 3 de la proposition précédente sont satisfaites.

Démonstration. - Comme $\mathscr{C}$ a un objet initial, tout foncteur constant de Mod- $\mathscr{C}$ en un $\mathbb{k}$-module injectif est injectif et représente les morphismes de l'évaluation en 0 vers ledit module. Par conséquent,

$$
\operatorname{Ext}_{\mathscr{C} \text { op }}^{r}(M, N) \simeq \operatorname{Ext}_{\mathbb{k}}^{r}(M, N)
$$

lorsque $M$ et $N$ sont des $\mathbb{k}$-modules, vus dans le terme de gauche comme foncteurs constants depuis $\mathscr{C}^{\mathrm{op}}$. Ces groupes sont donc nuls pour $r \geq 2$.

Soit $C$. le complexe de Mod- $\mathscr{C}$ obtenu en prenant les co-invariants par rapport à $G_{\infty}$ d'une résolution projective de $\mathbb{k} \in \operatorname{Ob} \operatorname{Mod}-\left(\mathscr{C} \times G_{\infty}\right)$. La deuxième suite spectrale de la proposition 2.16 s'obtient en prenant le produit tensoriel au-dessus de $\mathscr{C}$ de $C$ • et d'une résolution projective de $F$. Les propositions A.1 (appendice A) et 2.25 donnent alors la conclusion.

On peut également utiliser l'autre suite spectrale pour l'homologie de la catégorie $\mathscr{C} \times G_{\infty}$ donnée par la proposition 2.16, par l'intermédiaire de la propriété générale suivante :

Proposition 2.27. - Soient $\mathscr{G}$ et $\mathscr{B}$ deux petites catégories, $\Pi: \mathscr{G} \times \mathscr{B} \rightarrow \mathscr{C}$ la projection et $F$ un objet de $\mathscr{G}$-Mod. On suppose que l'anneau $\mathbb{k}$ est de dimension homologique au plus 1.

Alors la suite spectrale

$$
E_{p, q}^{2}(F)=H_{p}\left(\mathscr{B} ; H_{q}(\mathscr{Q} ; F)\right) \Rightarrow H_{p+q}\left(\mathscr{G} \times \mathscr{B} ; \Pi^{*} F\right)
$$

donnée par la proposition 2.16 (où $H_{q}(\mathscr{G} ; F)$ est vu comme objet constant de $\mathcal{B}$-Mod) s'effondre à la deuxième page.

De surcroît, cette suite spectrale induit un scindement (non nécessairement naturel en $F$ )

$$
H_{n}\left(\mathscr{G} \times \mathscr{B} ; \Pi^{*} F\right) \simeq \bigoplus_{p+q=n} H_{p}\left(\mathscr{B} ; H_{q}(\mathscr{Q} ; F)\right)
$$


Démonstration. - On suit la démarche de la section 5 de l'article «Stable $K$-theory is bifunctor homology » de Franjou et Pirashvili, dans le volume [13], qui elle-même s'inspire du théorème 2 de l'article [6] de Betley et Pirashvili.

Comme dans [13], on remarque que cette suite spectrale est une $\partial$-suite spectrale (cf. 2.2 de l'article de Franjou-Pirashvili), c'est-à-dire que, pour toute suite exacte courte $0 \rightarrow N \rightarrow P \rightarrow F \rightarrow 0$ de $\mathscr{G}$-Mod et tous entiers $r \geq 2$, $p$ et $q$, on dispose d'un morphisme $\partial^{r}: E_{p, q}^{r}(F) \rightarrow E_{p, q-1}^{r}(N)$ de sorte que $\partial^{r+1}$ est le morphisme induit par $\partial^{r}$ en homologie et que $\partial$ commute à la différentielle de la suite spectrale. Les morphismes $\partial^{2}$ sont induits par le morphisme de liaison $H_{q}(\mathscr{Q} ; F) \rightarrow H_{q-1}(\mathscr{G} ; N)$; la structure de $\partial$-suite spectrale s'obtient en l'étendant aux complexes de chaînes sur $\mathscr{C}$-Mod (i.e. en passant à la catégorie dérivée).

On choisit ensuite une suite exacte courte $0 \rightarrow N \rightarrow P \rightarrow F \rightarrow 0$ avec $P$ projectif : le morphisme de liaison $H_{q}(\mathscr{Q} ; F) \rightarrow H_{q-1}(\mathscr{G} ; N)$ est un isomorphisme pour $q \geq 1$ et un monomorphisme pour $q=1$, lequel est scindé parce que son conoyau est un sous-module du module projectif $H_{0}(\mathscr{G} ; P)$ (si $P$ est un projectif standard $P_{a}^{\mathscr{Q}}, H_{0}(\mathscr{G} ; P) \simeq \mathbb{k}$ ), donc est lui-même projectif par l'hypothèse faite sur $\mathbb{k}$. Par conséquent, $\partial^{2}: E_{p, q}^{2}(F) \rightarrow E_{p, q-1}^{2}(N)$ est injectif, ce qui nous permet d'appliquer le lemme 2.2 de l'article de Franjou-Pirashvili susmentionné pour conclure à l'effondrement de la suite spectrale à la deuxième page.

Le scindement se démontre de façon analogue, mais indépendante, par récurrence sur $n$ (on s'inspire ici directement de [6]) : il est immédiat pour $n=0$; on suppose donc $n>0$ et l'on se donne comme avant une suite exacte $0 \rightarrow N \rightarrow P \rightarrow F \rightarrow 0$ avec $P$ projectif, qui induit des isomorphismes $H_{i}(\mathscr{G} ; F) \simeq H_{i-1}(\mathscr{G} ; N)$ pour $i>1$ et un scindement (non naturel) $H_{0}(\mathscr{G} ; N) \simeq K \oplus H_{1}(\mathscr{G} ; F)$, où $K=K e r\left(H_{0}(\mathscr{G} ; P) \rightarrow H_{0}(\mathscr{G} ; F)\right)$ est un $\mathbb{k}$-module projectif. On considère alors le diagramme

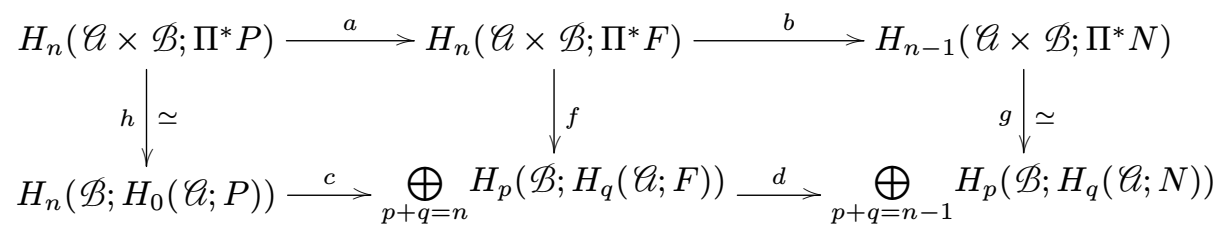

dans lequel :

1. la ligne supérieure est une partie de la suite exacte longue du foncteur homologique $H_{*}(\mathscr{G} \times \mathcal{B} ;-)$;

2. $h$ est le «coin» de la suite spectrale $\left(E_{p, q}^{r}(P)\right)$, qui est un isomorphisme parce que $E_{p, q}^{2}(P)=0$ pour $q>0, P$ étant projectif;

3. $g$ est un isomorphisme donné par l'hypothèse de récurrence;

4. $f$ est le morphisme dont la composante $H_{n}\left(\mathscr{G} \times \mathcal{B} ; \Pi^{*} F\right) \rightarrow H_{n}\left(\mathscr{B} ; H_{0}(\mathscr{C} ; F)\right)$ est le coin de la suite spectrale et la composante $H_{n}\left(\mathscr{G} \times \mathscr{B} ; \Pi^{*} F\right) \rightarrow H_{n-i}\left(\mathscr{B} ; H_{i}(\mathscr{C} ; F)\right)$ est pour $i>0$ composée de

$H_{n}\left(\mathscr{C} \times \mathscr{B} ; \Pi^{*} F\right) \stackrel{b}{\rightarrow} H_{n-1}\left(\mathscr{C} \times \mathscr{B} ; \Pi^{*} N\right) \stackrel{g}{\rightarrow} \bigoplus_{p+q=n-1} H_{p}\left(\mathscr{B} ; H_{q}(\mathscr{C} ; N) \rightarrow H_{n-i}\left(\mathscr{B} ; H_{i-1}(\mathscr{C} ; N)\right)\right.$ et de la flèche induite par l'isomorphisme $H_{i}(\mathscr{G} ; F) \simeq H_{i-1}(\mathscr{G} ; N)$ pour $i>1$ et le monomorphisme scindé $H_{1}(\mathscr{G} ; F) \hookrightarrow K \oplus H_{1}(\mathscr{G} ; F) \simeq H_{0}(\mathscr{G} ; N)$ pour $i=1$; 
5. le morphisme $d$ est défini de manière évidente pour que le carré de droite commute;

6. le morphisme $c$ est la composée du morphisme $H_{n}\left(\mathscr{B} ; H_{0}(\mathscr{Q} ; P)\right) \rightarrow H_{n}\left(\mathscr{B} ; H_{0}(\mathscr{Q} ; F)\right)$ induit par $P \rightarrow F$ et de l'inclusion canonique. Cela assure que le carré de gauche commute, par naturalité du coin de la suite spectrale et par nullité de la composée $b a$.

L'exactitude de la suite $H_{n}\left(\mathscr{B} ; H_{0}(\mathscr{G} ; P)\right) \rightarrow H_{n}\left(\mathscr{B} ; H_{0}(\mathscr{G} ; F)\right) \rightarrow H_{n-1}(\mathscr{B} ; K)$ déduite de la suite exacte courte $0 \rightarrow K \rightarrow H_{0}(\mathscr{G} ; P) \rightarrow H_{0}(\mathscr{G} ; F) \rightarrow 0$ permet de voir que la ligne inférieure du diagramme est exacte.

En notant $H_{i}(A)$ pour $H_{i}(\mathscr{G} \times \mathscr{B} ; A)$ et $g r_{i} H(A)$ pour $\underset{r+s=i}{\bigoplus_{r}} H_{r}\left(\mathscr{B} ; H_{s}(\mathscr{G} ; A)\right)$ pour alléger, le lemme des cinq appliqué au diagramme commutatif aux lignes exactes (déduit de l'hypothèse de récurrence)

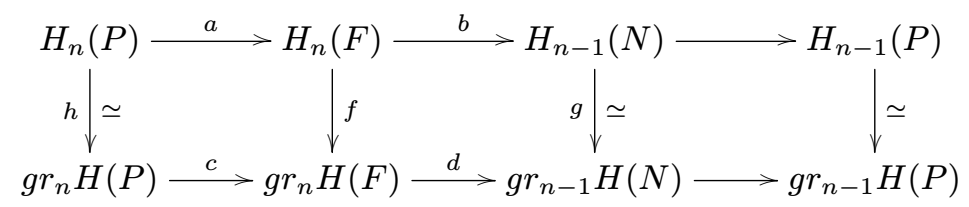

montre que $f$ est surjectif.

On considère à présent un diagramme

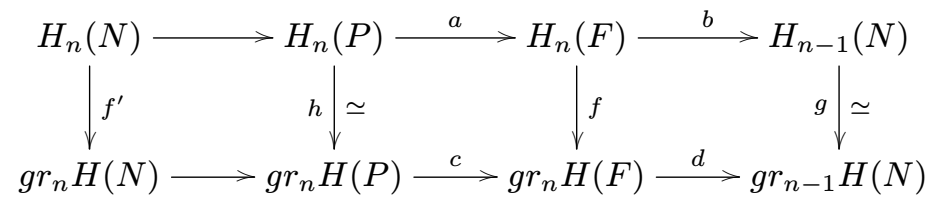

dans lequel la flèche $f^{\prime}$ est construite comme la flèche $f$, en remplaçant $F$ par $N$. Ce diagramme est commutatif et ses lignes sont exactes, pour des raisons similaires aux précédentes. Ce qu'on vient d'établir montre que la flèche $f^{\prime}$ est surjective. Le lemme des cinq montre alors que $f$ est injective, ce qui achève la démonstration.

En particulier, sous les hypothèses de la proposition 2.26, on obtient une suite spectrale fonctorielle

$$
E_{p, q}^{2}(F)=H_{p}\left(G_{\infty} ; H_{q}(\mathscr{C} ; F)\right) \Rightarrow H_{p+q}\left(\mathscr{C} \times G_{\infty} ; \Pi^{*} F\right) \simeq H_{p+q}\left(G_{\infty} ; F_{\infty}\right),
$$

où $G_{\infty}$ opère trivialement sur $H_{q}(\mathscr{C} ; F)$, qui s'effondre à la deuxième page et induit un scindement a priori non naturel

$$
H_{n}\left(G_{\infty} ; F_{\infty}\right) \simeq \bigoplus_{p+q=n} H_{p}\left(G_{\infty} ; H_{q}(\mathscr{C} ; F)\right)
$$

Remarque 2.28. - L'hypothèse que $\mathscr{C}$ possède un objet initial n'est pas nécessaire pour cette suite spectrale. On notera par ailleurs que, même dans les cas usuels favorables, il n'existe généralement pas de scindement naturel de la filtration associée à cette suite spectrale (contrairement à ce que la rédaction de l'article [6] peut laisser penser). 
Les suites spectrales que nous venons d'étudier, qui convergent vers $H_{*}\left(G_{\infty} ; F_{\infty}\right)$, qui s'arrêtent au terme $E^{2}$ dans les cas favorables, méritent d'être comparées à celle obtenue en $K$-théorie stable (et avec ses variantes faisant intervenir d'autres groupes que le groupe linéaire).

Rappelons sa construction. Soit $G$ un groupe dont le sous-groupe des commutateurs est parfait (c'est le cas du groupe linéaire infini sur un anneau arbitraire, mais aussi du groupe symétrique infini, du groupe orthogonal ou symplectique infini sur un corps commutatif). On applique la construction plus de Quillen au classifiant $B G$ de $G$ (comme groupe discret) et on forme la fibre homotopique $F_{G}$ de l'application canonique $B G \rightarrow B G^{+}$.

L'homologie de $F_{G}$ à coefficients dans un $G$-module $M$, vu comme $\pi_{1}\left(F_{G}\right)$-module via le morphisme canonique $\pi_{1}\left(F_{G}\right) \rightarrow \pi_{1}(B G)=G$, est par définition la $K$-théorie stable $G$-généralisée à coefficients dans $M$; on la note $K_{*}^{s}(G ; M)$. Le point remarquable est le suivant : dans la suite spectrale de Serre

$$
E_{p, q}^{2}=H_{p}\left(B G^{+} ; K_{q}^{s}(G ; M)\right) \simeq H_{p}\left(G ; K_{q}^{s}(G ; M)\right) \Rightarrow H_{p+q}(G ; M)
$$

l'action du groupe $G$ sur le groupe abélien $K_{q}^{s}(G ; M)$ est triviale (cf. par exemple [19], théorème 3.1).

De plus, dans de nombreux cas, la suite spectrale s'effondre au terme $E^{2}$ et la filtration associée se scinde (de manière généralement non naturelle) : voir l'article [6] et l'article «Stable $K$-theory is bifunctor homology » de Franjou et Pirashvili, déjà évoqués, pour le cas classique du groupe linéaire (nous n'avons d'ailleurs fait que reprendre leur démonstration pour établir la proposition 2.27); ce cas est adapté dans [5], théorème 1.3, pour le groupe symétrique (qui comme l'article [6] peut suggérer un scindement naturel, incorrect, de la graduation).

La $K$-théorie stable comme l'homologie de catégorie rendent donc généralement des services très analogues en terme de calcul de l'homologie du groupe $G$ à coefficients tordus : dans les cas où l'on sait les identifier naturellement, la suite spectrale (4) et celle de la proposition 2.27 sont isomorphes. Néanmoins, nous ne connaissons pas d'analogue à la suite spectrale de la proposition 2.19 (qui présente l'avantage non seulement de s'effondrer à la deuxième page, mais aussi de procurer une décomposition fonctorielle de l'homologie stable dans les cas favorables, contrairement à celle de la proposition 2.27).

Il convient d'ailleurs de noter que les arguments de Scorichenko pour identifier la $K$-théorie stable d'un anneau quelconque et l'homologie de la catégorie des modules projectifs de type fini pour des coefficients déduits de bifoncteurs polynomiaux n'utilisent pas réellement la définition de la $K$-théorie stable, mais seulement l'existence de la suite spectrale naturelle (4) et d'un morphisme naturel gradué $K_{*}^{s}\left(G L_{\infty} ; M\right) \rightarrow H_{*}\left(G L_{\infty} ; M\right)$ (induit par l'application canonique $F_{G} \rightarrow B G$ ). On déduit de ce morphisme un morphisme naturel de la $K$-théorie stable à coefficients provenant d'un bifoncteur vers l'homologie de ce bifoncteur, morphisme dont Scorichenko montre qu'il est bijectif si le bifoncteur est polynomial en exploitant uniquement la suite spectrale (4). Dans le cas d'un corps fini, l'homologie du groupe linéaire infini à coefficients dans le corps est trivial, comme l'a montré Quillen dans [25], de sorte que la suite spectrale (4) se réduit à un isomorphisme naturel entre $K$-théorie stable et homologie de $G L_{\infty}$; c'est pourquoi le théorème de Scorichenko sur la $K$-théorie stable se réduit dans ce cas, traité auparavant par Betley (cf. [4]) et Suslin (cf. l'appendice de [14]) 
indépendamment, à une identification entre homologie du groupe linéaire (à coefficients tordus) et homologie de catégorie.

Nous reviendrons dans les appendices $\mathrm{F}$ et $\mathrm{E}$ sur l'équivalence des méthodes de calcul d'homologie stable des groupes linéaires et symétriques respectivement, à coefficients venant d'un foncteur convenable, à l'aide de la $K$-théorie stable (généralisée au groupe symétrique infini dans le second cas) ou à l'aide de notre suite spectrale utilisant directement l'homologie d'une catégorie appropriée.

\section{Cas des groupes orthogonaux et symplectiques}

Cette section fournit le résultat principal de cet article, qui relie l'homologie stable des groupes orthogonaux (resp. symplectiques) à coefficients tordus et des groupes de torsion sur la catégorie des espaces vectoriels. Ces derniers sont accessibles au calcul, dans les cas favorables, comme nous l'illustrons à la section 4.

On commence par identifier une certaine catégorie de fractions de $\mathcal{E}_{q}^{\mathrm{deg}}$ (resp. de $\left.\mathcal{E}_{\text {alt }}^{\mathrm{deg}}\right)$ à une catégorie de Burnside (cf. définition C.4). La décomposition des foncteurs de Mackey non additifs associés à cette catégorie (cf. théorème C.5) intervient pour simplifier la deuxième page de la suite spectrale de la section précédente pour les groupes orthogonaux ou symplectiques. Combiné à des résultats d'annulation en homologie des foncteurs, cela permet d'obtenir notre résultat central, le théorème 3.21.

\subsection{Les catégories de fractions $\mathcal{E}_{q}^{\mathrm{deg}}\left[(-\perp \mathbf{H})^{-1}\right]$ et $\mathcal{E}_{\mathrm{alt}}^{\mathrm{deg}}\left[(-\perp \mathbf{H})^{-1}\right]$}

Dans le cas où $\mathscr{C}=\mathcal{E}_{q}^{\mathrm{deg}}(k)$ (resp. $\mathscr{C}=\mathcal{E}_{\mathrm{alt}}^{\mathrm{deg}}(k)$ ), où $k$ est un corps commutatif fixé, le foncteur $\mathbb{L}_{n} Q_{!}(\mathbb{k})$ transforme l'inclusion canonique $D \rightarrow S_{\mathbf{H}}(i) \perp D=\mathbf{H}^{\perp i} \perp D$ en un isomorphisme d'après la proposition 2.22. Cette observation justifie que l'on s'intéresse dans ce paragraphe à la catégorie de fractions inversant les morphismes canoniques de la forme $D \stackrel{i}{\rightarrow} D \perp \mathbf{H}^{\perp n}$.

Soient $(-\perp \mathbf{H})$ l'ensemble de flèches de $\mathcal{E}_{q}^{\text {deg }}$ suivant :

$$
(-\perp \mathbf{H})=\{D \stackrel{i}{\rightarrow} D \perp H \mid H \text { espace hyperbolique } ; i \text { inclusion canonique }\}
$$

et $\varepsilon_{q}^{\operatorname{deg}}\left[(-\perp \mathbf{H})^{-1}\right]$ la catégorie de fractions correspondante (qui existe par des résultats généraux, voir par exemple [18]). On note $\phi: \mathcal{E}_{q}^{\mathrm{deg}} \rightarrow \mathcal{E}_{q}^{\mathrm{deg}}\left[(-\perp \mathbf{H})^{-1}\right]$ le foncteur canonique. Le but de ce paragraphe est de démontrer qu'il existe une équivalence de catégories

$$
\Psi: \mathcal{E}_{q}^{\mathrm{deg}}\left[(-\perp \mathbf{H})^{-1}\right] \stackrel{\simeq}{\longrightarrow} \operatorname{Sp}\left(\mathcal{E}_{\mathrm{inj}}^{f}\right)
$$

où $\mathcal{E}_{\text {inj }}^{f}$ est la sous-catégorie des injections de $\mathcal{E}^{f}(k)$ (cf. définition D.1) et $\mathrm{Sp}($.$) désigne la ca-$ tégorie de Burnside dont on rappelle la construction dans la définition C.4 de l'appendice C.

Après avoir donné quelques résultats essentiels sur les morphismes de $\mathcal{E}_{q}^{\mathrm{deg}}\left[(-\perp \mathbf{H})^{-1}\right]$, on définira le foncteur $\Psi$, puis on montrera que $\Psi$ est essentiellement surjectif, plein et fidèle.

Notation 3.1. - Dans les diagrammes dans la catégorie de fractions $\mathcal{E}_{q}^{\mathrm{deg}}\left[(-\perp \mathbf{H})^{-1}\right]$, on notera par $\leadsto$ les morphismes de $\delta_{q}^{\text {deg }}$ qui sont éléments de $(-\perp \mathbf{H})$ et par $\rightarrow$ tout morphisme de $\varepsilon_{q}^{\text {deg }}$. 
Remarquons que $(-\perp \mathbf{H})$ est stable par composition et que pour tout objet $D$ de $\mathcal{E}_{q}^{\text {deg }}$, $\operatorname{Id}_{D} \in(-\perp \mathbf{H})$. On a également la propriété suivante :

Lemme 3.2. - Soient $H$ un espace hyperbolique et $f: D \rightarrow D^{\prime}$ un morphisme de $\mathcal{E}_{q}^{\mathrm{deg}}$; il existe $g: D \perp H \rightarrow D^{\prime} \perp H$ rendant le diagramme suivant commutatif :

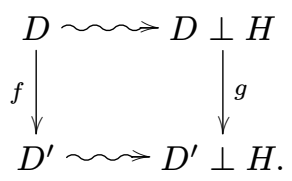

Démonstration. - Ce diagramme est commutatif pour $g=f \perp \operatorname{Id}_{H}$.

Ce lemme nous permet de donner la description suivante des morphismes de $\mathcal{E}_{q}^{\operatorname{deg}}\left[(-\perp \mathbf{H})^{-1}\right]$

Lemme 3.3. - Tout morphisme de $\mathcal{E}_{q}^{\mathrm{deg}}\left[(-\perp \mathbf{H})^{-1}\right]$ s'écrit sous la forme $g^{-1} f$ où g est un élément de $(-\perp \mathbf{H})$ et $f$ est un morphisme de $\mathcal{E}_{q}^{\mathrm{deg}}$.

Remarque 3.4. - L'ensemble $(-\perp \mathbf{H})$ n'admet pas un «calcul à gauche des fractions » au sens de Gabriel et Zisman ([18]). En effet, pour $D \stackrel{i}{\longrightarrow} D \perp H$ un élément de $(-\perp \mathbf{H})$, $f: D \perp H \rightarrow D^{\prime}$ et $g: D \perp H \rightarrow D^{\prime}$ deux morphismes de $\mathcal{E}_{q}^{\text {deg }}$ tels que $f i=g i$, il n'existe pas de morphisme de $(-\perp \mathbf{H}) \quad D^{\prime} \stackrel{i^{\prime}}{\sim} D^{\prime} \perp K$ tel que $i^{\prime} f=i^{\prime} g$. Nous n'avons donc pas une description de $\mathcal{E}_{q}^{\mathrm{deg}}\left[(-\perp \mathbf{H})^{-1}\right]$ aussi simple que dans le cas considéré par GabrielZisman ; il est donc a priori difficile de savoir quand deux morphismes sont égaux dans cette catégorie, l'écriture d'un morphisme de $\mathcal{E}_{q}^{\mathrm{deg}}\left[(-\perp \mathbf{H})^{-1}\right]$ sous la forme $g^{-1} f$ n'étant pas unique. Néanmoins nous avons les résultats suivants qui seront essentiels dans la preuve de la fidélité du foncteur $F$ du théorème 3.17.

Lemme 3.5. - Soient $D$ un objet de $\varepsilon_{q}^{\mathrm{deg}}, H$ un espace hyperbolique et $f \in \operatorname{Aut}_{\mathscr{E}_{q}^{\operatorname{deg}}}(D \perp H)$ tel que $f_{\mid D}=\operatorname{Id}_{D}$; alors $f=\operatorname{Id}_{D \perp H}$ dans $\mathcal{E}_{q}^{\mathrm{deg}}\left[(-\perp \mathbf{H})^{-1}\right]$.

Démonstration. - Soient $D, H$ et $f$ comme dans l'énoncé. L'inclusion canonique $D \stackrel{i}{\rightarrow} D \perp H$ est un élément de $(-\perp \mathbf{H})$ et est donc un isomorphisme dans $\varepsilon_{q}^{\operatorname{deg}}\left[(-\perp \mathbf{H})^{-1}\right]$. Or on a : $f i=i$, dont on déduit que $f=\operatorname{Id}_{D \perp H}$ dans $\mathcal{E}_{q}^{\mathrm{deg}}\left[(-\perp \mathbf{H})^{-1}\right]$.

Le cas particulier où $D$ est l'espace vectoriel nul fournit le lemme suivant :

Lemme 3.6. - Soient $H$ un espace hyperbolique et $f \in \operatorname{Aut}_{\mathscr{G}_{q}^{\operatorname{deg}}}(H)$. On a alors $f=\operatorname{Id}_{H}$ dans $\varepsilon_{q}^{\mathrm{deg}}\left[(-\perp \mathbf{H})^{-1}\right]$.

On déduit de ce lemme le résultat suivant :

Proposition 3.7. - Soient $D$ un objet de $\varepsilon_{q}^{\mathrm{deg}}, H$ un espace hyperbolique et $f, g \in \operatorname{Hom}_{\delta_{q}^{\mathrm{deg}}}(D, H)$. Alors $f=g$ dans $\mathcal{E}_{q}^{\mathrm{deg}}\left[(-\perp \mathbf{H})^{-1}\right]$.

Démonstration. - Par le théorème de Witt, il existe $h \in \operatorname{Aut}_{\mathscr{E}_{q}^{\operatorname{deg}}}(H)$ tel que $h f=g$. Le lemme 3.6 permet d'en déduire que $f=g$ dans $\mathscr{E}_{q}^{\text {deg }}\left[(-\perp \mathbf{H})^{-1}\right]$. 
Nous aurons également besoin dans la suite du lemme très facile suivant :

Lemme 3.8. - Soient $\alpha, \beta: V \rightarrow W$ dans $\mathcal{E}_{q}^{\mathrm{deg}}\left[(-\perp \mathbf{H})^{-1}\right]$. Alors il existe un espace hyperbolique $H$ tel que $\alpha=i^{-1}$ f et $\beta=i^{-1}$ avec $f, g: V \rightarrow W \perp H$ et $i: W \rightarrow W \perp H$ l'inclusion canonique.

Démonstration. - D'après le lemme 3.3, on a : $\alpha=i_{1}^{-1} f_{1}$ et $\beta=i_{2}^{-1} g_{2}$ où $f_{1}: V \rightarrow W \perp H_{1}, g_{2}: V \rightarrow W \perp H_{2}$ et $i_{1}: W \rightarrow W \perp H_{1}, i_{2}: W \rightarrow W \perp H_{2}$ sont les inclusions canoniques. Il suffit alors de vérifier que $\alpha=i_{2}^{-1} i_{2} i_{1}^{-1} f_{1}=i^{-1} f$ où $f: V \rightarrow W \perp H_{1} \perp H_{2}$ est la composée de $f_{1}$ et de l'inclusion canonique $W \perp H_{1} \rightarrow W \perp H_{1} \perp H_{2}$. De même pour $\beta$.

Notation 3.9. - Dans la suite, un morphisme de $\mathcal{E}_{q}^{\mathrm{deg}}\left[(-\perp \mathbf{H})^{-1}\right]$ se décomposant sous la forme $\alpha=i^{-1} f$, où $f: V \rightarrow W \perp H$ et $i: W \rightarrow W \perp H$ l'inclusion canonique, sera noté :

$$
V \stackrel{f}{\longrightarrow} W \perp H \varkappa W .
$$

Remarque 3.10. - La catégorie $\mathcal{E}_{q}^{\mathrm{deg}}\left[(-\perp \mathbf{H})^{-1}\right]$ est équivalente à la catégorie de fractions de $\varepsilon_{q}^{\text {deg }}$ où l'on inverse l'ensemble des inclusions canoniques $D \rightarrow D \perp K$ où $K$ est un espace quadratique non dégénéré. En effet, comme tout espace quadratique non dégénéré $K$ se plonge dans un espace hyperbolique $K \perp K^{\prime}$, la composée suivante :

$$
V \stackrel{a}{\rightarrow} V \perp K \stackrel{b}{\rightarrow} V \perp K \perp K^{\prime}
$$

est inversible dans $\delta_{q}^{\mathrm{deg}}\left[(-\perp \mathbf{H})^{-1}\right]$, ce qui implique que $a$ est inversible à gauche et $b$ est inversible à droite dans $\mathcal{E}_{q}^{\mathrm{deg}}\left[(-\perp \mathbf{H})^{-1}\right]$. De plus, comme la composée

$$
V \perp K \stackrel{b}{\rightarrow} V \perp K \perp K^{\prime} \stackrel{c}{\rightarrow} V \perp K \perp K^{\prime} \perp K
$$

est inversible dans $\mathcal{E}_{q}^{\mathrm{deg}}\left[(-\perp \mathbf{H})^{-1}\right], b$ est inversible à gauche dans $\mathcal{E}_{q}^{\mathrm{deg}}\left[(-\perp \mathbf{H})^{-1}\right]$. On en déduit que $b$ est inversible dans $\mathcal{E}_{q}^{\mathrm{deg}}\left[(-\perp \mathbf{H})^{-1}\right]$ et donc que $a$ l'est également.

Afin de définir le foncteur de $\mathcal{E}_{q}^{\text {deg }}$ dans $\operatorname{Sp}\left(\mathcal{E}_{\mathrm{inj}}^{f}\right)$ dont découle l'équivalence de catégories annoncée en début de section, nous avons besoin du lemme facile suivant.

Lemme 3.11. - Soit $f \in \operatorname{Hom}_{\mathscr{E}_{q}^{\operatorname{deg}}}\left(D, D^{\prime}\right)$. On a $f^{-1}\left(\operatorname{Rad}\left(D^{\prime}\right)\right) \subset \operatorname{Rad}(D)$ où $\operatorname{Rad}$ désigne le radical.

Proposition 3.12. - Il existe un foncteur $\widetilde{\Psi}: \mathcal{E}_{q}^{\mathrm{deg}} \rightarrow \mathrm{Sp}\left(\mathcal{E}_{\mathrm{inj}}^{f}\right)$ défini sur les objets par $\widetilde{\Psi}(D)=\operatorname{Rad}(D)$ et sur les morphismes par :

$$
\widetilde{\Psi}\left(D \stackrel{f}{\rightarrow} D^{\prime}\right)=\operatorname{Rad}(D) \stackrel{i}{\leftarrow} f^{-1}\left(\operatorname{Rad}\left(D^{\prime}\right)\right) \stackrel{\widetilde{f}}{\rightarrow} \operatorname{Rad}\left(D^{\prime}\right)
$$

où $i$ est l'inclusion obtenue dans le lemme 3.11 et $\tilde{f}$ est la restriction de $f \grave{a} f^{-1}\left(\operatorname{Rad}\left(D^{\prime}\right)\right)$. 
Démonstration. - Vérifions que $\widetilde{\Psi}$ définit bien un foncteur.

Pour $D \in \mathcal{E}_{q}^{\mathrm{deg}}: \widetilde{\Psi}(D \stackrel{\mathrm{Id}}{\longrightarrow} D)=\operatorname{Rad}(D) \stackrel{\mathrm{Id}}{\longleftarrow} \operatorname{Rad}(D) \stackrel{\mathrm{Id}}{\longrightarrow} \operatorname{Rad}(D)$.

Pour une paire de flèches composables de $\mathcal{E}_{q}^{\text {deg }}: D \stackrel{f}{\rightarrow} D^{\prime} \stackrel{g}{\rightarrow} D^{\prime \prime}$, on a :

$$
\widetilde{\Psi}(g \circ f)=\operatorname{Rad}(D) \hookleftarrow(g \circ f)^{-1}\left(\operatorname{Rad}\left(D^{\prime \prime}\right)\right) \stackrel{\widetilde{g \circ f}}{\longrightarrow} \operatorname{Rad}\left(D^{\prime \prime}\right)
$$

et $\widetilde{\Psi}(g) \circ \widetilde{\Psi}(f)$ est donné par le diagramme en escalier suivant :

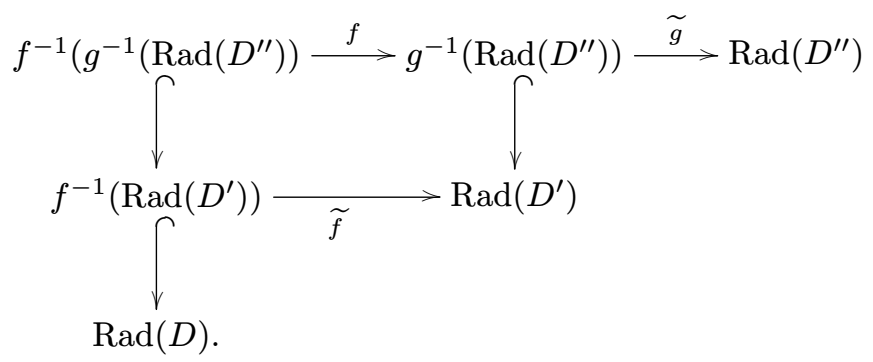

D'où

$$
\widetilde{\Psi}(g \circ f)=\widetilde{\Psi}(g) \circ \widetilde{\Psi}(f) .
$$

Proposition 3.13. - Le foncteur $\widetilde{\Psi}: \mathcal{E}_{q}^{\mathrm{deg}} \rightarrow \mathrm{Sp}\left(\mathcal{E}_{\mathrm{inj}}^{f}\right)$ induit un unique foncteur $\Psi: \mathcal{E}_{q}^{\mathrm{deg}}\left[(-\perp \mathbf{H})^{-1}\right] \rightarrow \mathrm{Sp}\left(\mathcal{E}_{\mathrm{inj}}^{f}\right)$ rendant le diagramme suivant commutatif :

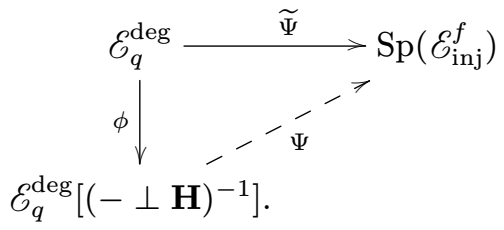

Démonstration. - Soit $D \stackrel{i}{\rightarrow} D \perp H$ un élément de $(-\perp \mathbf{H})$; on a

$$
\widetilde{\Psi}(i)=\operatorname{Rad}(D) \stackrel{\mathrm{Id}}{\longleftarrow} \operatorname{Rad}(D) \stackrel{\mathrm{Id}}{\longrightarrow} \operatorname{Rad}(D)
$$

(qui est bien un isomorphisme de $\operatorname{Sp}\left(\mathcal{E}_{\text {inj }}^{f}\right)$ !).

Par la propriété universelle de la catégorie des fractions, il existe un unique foncteur $\Psi: \mathcal{E}_{q}^{\mathrm{deg}}\left[(-\perp \mathbf{H})^{-1}\right] \rightarrow \operatorname{Sp}\left(\mathcal{E}_{\mathrm{inj}}^{f}\right)$ tel que $\Psi \circ \phi=\widetilde{\Psi}$.

Afin de montrer que le foncteur $\Psi$ fournit une équivalence de catégories, on prouve dans la suite qu'il est essentiellement surjectif et pleinement fidèle.

Proposition 3.14. - Le foncteur $\Psi: \mathcal{E}_{q}^{\mathrm{deg}}\left[(-\perp \mathbf{H})^{-1}\right] \rightarrow \operatorname{Sp}\left(\mathcal{E}_{\mathrm{inj}}^{f}\right)$ est essentiellement surjectif.

Démonstration. - Soit $V \in \mathcal{E}_{\text {inj }}^{f}$; on munit $V$ de la forme quadratique nulle. L'espace quadratique $D$ ainsi obtenu étant totalement isotrope, on a $\Psi(D)=V$.

Proposition 3.15. - Le foncteur $\Psi: \mathcal{E}_{q}^{\mathrm{deg}}\left[(-\perp \mathbf{H})^{-1}\right] \rightarrow \operatorname{Sp}\left(\mathcal{E}_{\mathrm{inj}}^{f}\right)$ est plein. 
Démonstration. - Soient $V=\operatorname{Rad}(V) \perp H$ et $W=\operatorname{Rad}(W) \perp K$ deux objets de $\mathcal{E}_{q}^{\operatorname{deg}}\left[(-\perp \mathbf{H})^{-1}\right]$ et $f \in \operatorname{Hom}_{\operatorname{Sp}\left(\mathcal{E}_{\text {inj }}^{f}\right)}(\operatorname{Rad}(V), \operatorname{Rad}(W))$. Par définition des morphismes de $\operatorname{Sp}\left(\varepsilon_{\text {inj }}^{f}\right)$, il existe un sous-espace vectoriel $X$ de $\operatorname{Rad}(V)$ tel que $f=\operatorname{Rad}(V) \stackrel{i}{\leftarrow} X \stackrel{\beta}{\rightarrow} \operatorname{Rad}(W)$, où $i$ est l'inclusion et $\beta$ est une injection linéaire.

Soient $X^{\prime}$ un espace vectoriel supplémentaire de $X$ dans $\operatorname{Rad}(V)$ et $X^{\prime \prime}$ un supplémentaire de $\beta(X)$ dans $\operatorname{Rad}(W)$. Comme les formes quadratiques sur les radicaux sont nulles, on a :

$$
\operatorname{Rad}(V) \simeq X \perp X^{\prime} \quad \operatorname{Rad}(W) \simeq \beta(X) \perp X^{\prime \prime} .
$$

Par les propriétés générales de la catégorie $\mathcal{E}_{q}^{\mathrm{deg}}$, il existe un espace hyperbolique $L$ et $g \in \operatorname{Hom}_{\mathscr{E}_{q}^{\operatorname{deg}}}\left(X^{\prime}, L\right)$.

Soit $k: V \simeq X \perp X^{\prime} \perp H \rightarrow\left(\beta(X) \perp X^{\prime \prime} \perp K\right) \perp H \perp L \simeq W \perp H \perp L$ l'application donnée par la matrice :

$$
\left(\begin{array}{ccc}
\beta & 0 & 0 \\
0 & 0 & \operatorname{Id}_{H} \\
0 & g & 0
\end{array}\right) .
$$

L'application $u=V \stackrel{k}{\longrightarrow} W \perp H \perp L \varkappa W$ de $\mathcal{E}_{q}^{\text {deg }}\left[\left(\begin{array}{lll}- & \perp & \mathbf{H}\end{array}\right)^{-1}\right]$ vérifie $\Psi(u)=f$.

Proposition 3.16. - Le foncteur $\Psi: \mathcal{E}_{q}^{\mathrm{deg}}\left[(-\perp \mathbf{H})^{-1}\right] \rightarrow \mathrm{Sp}\left(\mathcal{E}_{\mathrm{inj}}^{f}\right)$ est fidèle.

Démonstration. - Soient $\quad \alpha, \beta \in \operatorname{Hom}_{\dot{E}_{q}^{\mathrm{deg}}\left[(-\perp \mathbf{H})^{-1}\right]}(V, W)$ tels que $\Psi(\alpha)=\Psi(\beta)$. D'après les lemmes 3.3 et 3.8 , on peut écrire $\alpha$ et $\beta$ sous la forme :

$$
\alpha=V \stackrel{f}{\longrightarrow} W \perp H \varkappa W ; \quad \beta=V \stackrel{g}{\longrightarrow} W \perp H \varkappa W .
$$

Par la définition de la catégorie de Burnside $\operatorname{Sp}\left(\mathcal{E}_{\text {inj }}^{f}\right)$ rappelée en C.4, l'égalité $\Psi(\alpha)=\Psi(\beta)$ implique que $f^{-1}(\operatorname{Rad}(W))=g^{-1}(\operatorname{Rad}(W))$ et $\widetilde{f}=\widetilde{g}$.

On a les décompositions suivantes des espaces $V$ et $W \perp H: V=f^{-1}(\operatorname{Rad}(W)) \perp D$ où $D$ est un objet de $\mathcal{E}_{q}^{\text {deg }} ; W \perp H=\operatorname{Rad}(W) \perp L$ où $L$ est un espace non dégénéré. Dans des bases de $V$ et $W \perp H$ obtenues en juxtaposant des bases de $f^{-1}(\operatorname{Rad}(W))$ et $D$ pour $V$ et de $\operatorname{Rad}(W)$ et $L$ pour $W \perp H$, la matrice de l'application $f$ s'écrit :

$$
\left(\begin{array}{ll}
\tilde{f} & \epsilon \\
0 & i
\end{array}\right)
$$

où $i: D \rightarrow L$ est une injection (préservant la forme quadratique) puisque $D \cap f^{-1}(\operatorname{Rad}(W))=0$, et $\epsilon: D \rightarrow \operatorname{Rad}(W)$ est une application linéaire. La matrice de $g$ dans la même base est de la forme

$$
\left(\begin{array}{ll}
\tilde{f} & \epsilon^{\prime} \\
0 & i^{\prime}
\end{array}\right)
$$

avec les mêmes conditions sur $i^{\prime}$ et $\epsilon^{\prime}$ que précédemment.

Comme $i$ et $i^{\prime}$ sont des injections préservant les formes quadratiques, à valeurs dans un espace quadratique non dégénéré, par le théorème de Witt, il existe $u \in O(L)$ tel que $u i=i^{\prime}$. De plus, comme $i$ est injective, il existe $\alpha: L \rightarrow \operatorname{Rad}(W)$ tel que $\epsilon+\alpha i=\epsilon^{\prime}$. 
Considérons l'automorphisme $l \operatorname{de} \operatorname{Rad}(W) \perp L$ de matrice

$$
\left(\begin{array}{cc}
\operatorname{Id} & \alpha \\
0 & u
\end{array}\right)
$$

l'application $l f$ a pour matrice

$$
\left(\begin{array}{cc}
f_{\mid f^{-1}(\operatorname{Rad}(W))} & \epsilon+\alpha i \\
0 & u i
\end{array}\right)=\left(\begin{array}{cc}
f_{\mid f^{-1}(\operatorname{Rad}(W))} \epsilon^{\prime} \\
0 & i^{\prime}
\end{array}\right)
$$

dont on déduit que $l f=g$. Or, par le lemme 3.5, on a $l=\operatorname{Id}_{\operatorname{Rad}(W) \perp L}$ d'où $: f=g$ dans $\mathcal{E}_{q}^{\mathrm{deg}}\left[(-\perp \mathbf{H})^{-1}\right]$.

Nous avons donc démontré le résultat suivant :

THÉORÈme 3.17. - Le foncteur $\widetilde{\Psi}: \mathcal{E}_{q}^{\mathrm{deg}} \rightarrow \mathrm{Sp}\left(\mathcal{E}_{\mathrm{inj}}^{f}\right)$ défini à la proposition 3.12 induit une équivalence de catégories

$$
\Psi: \mathcal{E}_{q}^{\mathrm{deg}}\left[(-\perp \mathbf{H})^{-1}\right] \stackrel{\simeq}{\longrightarrow} \operatorname{Sp}\left(\mathcal{E}_{\mathrm{inj}}^{f}\right)
$$

où $\varepsilon_{\mathrm{inj}}^{f}$ est la catégorie ayant pour objets les $k$-espaces vectoriels de dimension finie et pour morphismes les applications linéaires injectives.

Pour la catégorie $\mathcal{E}_{\mathrm{alt}}^{\mathrm{deg}}$, on démontre de manière similaire le théorème suivant.

THÉORÈme 3.18. - Le foncteur $\widetilde{\Psi}: \mathcal{E}_{\mathrm{alt}}^{\mathrm{deg}} \rightarrow \mathrm{Sp}\left(\mathcal{E}_{\mathrm{inj}}^{f}\right)$ défini comme à la proposition 3.12 mutatis mutandis induit une équivalence de catégories

$$
\Psi: \mathcal{E}_{\text {alt }}^{\text {deg }}\left[(-\perp \mathbf{H})^{-1}\right] \stackrel{\simeq}{\longrightarrow} \operatorname{Sp}\left(\mathcal{E}_{\text {inj }}^{f}\right)
$$

où $\mathbf{H}$ désigne l'espace symplectique défini en 1.9.2.

\subsection{Théorème fondamental}

On commence par donner quelques notations utilisées couramment dans la suite :

Notation 3.19. - Soit $k$ un corps commutatif.

1. On désigne par $O_{\infty}(k)\left(\right.$ resp. $\left.\operatorname{Sp}_{\infty}(k)\right)$ le groupe $\operatorname{colim}_{n \in \mathbb{N}} O_{n, n}(k)$ (resp. colim $\operatorname{Sp}_{n \in \mathbb{N}}(k)$ ), la colimite étant prise conformément aux conventions de la section 1.

2. On désigne par $S^{i}$ (resp. $\Gamma^{i}, \Lambda^{i}$ ) l'endofoncteur $i$-ième puissance symétrique (resp. divisée, extérieure) des $k$-espaces vectoriels.

3. On note $\mathcal{F}(k)$, voire $\mathcal{F}$, la catégorie $\mathcal{E}^{f}(k)$-Mod des foncteurs depuis les $k$-espaces vectoriels de dimension finie vers les $\mathbb{k}$-modules.

4. Lorsque $F$ est un foncteur depuis une catégorie d'espaces vectoriels, on note $F^{\vee}$ la précomposition de $F$ par le foncteur de dualité $(-)^{*}: F^{\vee}(V)=F\left(V^{*}\right)$. 
(On rappelle que $F^{*}$ désigne pour sa part, conformément à la notation de l'appendice A, la postcomposition de $F$ par la dualité, lorsque $\mathbb{k}$ est un corps.)

La notation $O_{\infty}$ est justifiée, pour les considérations homologiques qui sont les nôtres, par le fait que la colimite sur tous les groupes orthogonaux donne des résultats canoniquement isomorphes (même si le groupe orthogonal infini obtenu n'est pas isomorphe à celui qu'on considère) - cf. remarque 2.24.3.

On aura également besoin de la notion classique suivante :

Définition 3.20 (Cf. [13]). - Soit $k$ un corps.

1. Le foncteur différence de $\mathcal{F}(k)$ est l'endofoncteur $\Delta$ de cette catégorie défini comme étant le noyau de l'épimorphisme scindé évident $(-\oplus k)^{*} \rightarrow$ Id.

2. Un foncteur $F$ de $\mathcal{F}(k)$ est dit polynomial s'il existe $n \in \mathbb{N}$ tel que $\Delta^{n}(F)=0$; son degré est alors le plus grand entier $d$ tel que $\Delta^{d}(F) \neq 0$.

3. Un foncteur analytique est une colimite (qu'on peut supposer filtrante) de foncteurs polynomiaux.

Le théorème ci-dessous constitue le résultat principal de cet article.

THÉORÈme 3.21. - Soient $k$ un corps fini et $F$ un foncteur analytique de $\mathcal{F}(k)$.

Il existe des isomorphismes naturels gradués

$$
H_{*}\left(O_{\infty}(k) ; F_{\infty}\right) \simeq \operatorname{Tor}_{*}^{\mathcal{E}^{f}(k) \times O_{\infty}(k)}\left(\mathbb{k}\left[S^{2}\right]^{\vee}, F\right)
$$

et

$$
H_{*}\left(\operatorname{Sp}_{\infty}(k) ; F_{\infty}\right) \simeq \operatorname{Tor}_{*}^{\mathcal{E}^{f}(k) \times \operatorname{Sp}_{\infty}(k)}\left(\mathbb{k}\left[\Lambda^{2}\right]^{\vee}, F\right)
$$

(où les groupes $O_{\infty}(k)$ et $\mathrm{Sp}_{\infty}(k)$ agissent trivialement).

Corollaire 3.22. - Sous les hypothèses précédentes, il existe des suites spectrales naturelles en $F$ données par

$$
E_{p, q}^{2}=\operatorname{Tor}_{p}^{\varepsilon^{f}(k)}\left(H_{q}\left(O_{\infty}(k) ; \mathbb{k}\right) \underset{\mathbb{k}}{\otimes} \mathbb{k}\left[S^{2}\right]^{\vee}, F\right) \Rightarrow H_{p+q}\left(O_{\infty}(k) ; F_{\infty}\right)
$$

et

$$
E_{p, q}^{2}=\operatorname{Tor}_{p}^{\delta^{f}(k)}\left(H_{q}\left(\operatorname{Sp}_{\infty}(k) ; \mathbb{k}\right) \underset{\mathbb{k}}{\otimes \mathbb{k}}\left[\Lambda^{2}\right]^{\vee}, F\right) \Rightarrow H_{p+q}\left(\operatorname{Sp}_{\infty}(k) ; F_{\infty}\right) .
$$

Lorsque $\mathbb{k}$ est un anneau de dimension homologique au plus 1 , elles s'effondrent au terme $E^{2}$ et procurent des isomorphismes canoniques

$$
H_{n}\left(O_{\infty}(k) ; F_{\infty}\right) \simeq \bigoplus_{p+q=n} \operatorname{Tor}_{p}^{\delta^{f}(k)}\left(H_{q}\left(O_{\infty}(k) ; \mathbb{k}\right) \underset{\mathbb{k}}{\otimes \mathbb{k}}\left[S^{2}\right]^{\vee}, F\right)
$$

et

$$
H_{n}\left(\operatorname{Sp}_{\infty}(k) ; F_{\infty}\right) \simeq \bigoplus_{p+q=n} \operatorname{Tor}_{p}^{\delta^{f}(k)}\left(H_{q}\left(\operatorname{Sp}_{\infty}(k) ; \mathbb{k}\right) \underset{\mathbb{k}}{\otimes \mathbb{k}}\left[\Lambda^{2}\right]^{\vee}, F\right)
$$


Corollaire 3.23. - Sous les hypothèses du théorème, il existe des suites spectrales naturelles

$$
E_{p, q}^{2}=H_{p}\left(O_{\infty}(k) ; \operatorname{Tor}_{q}^{\mathcal{E}^{f}(k)}\left(\mathbb{k}\left[S^{2}\right]^{\vee}, F\right)\right) \Rightarrow H_{p+q}\left(O_{\infty}(k) ; F_{\infty}\right)
$$

et

$$
E_{p, q}^{2}=H_{p}\left(\operatorname{Sp}_{\infty}(k) ; \operatorname{Tor}_{q}^{\varepsilon^{f}(k)}\left(\mathbb{k}\left[\Lambda^{2}\right]^{\vee}, F\right)\right) \Rightarrow H_{p+q}\left(\operatorname{Sp}_{\infty}(k) ; F_{\infty}\right)
$$

qui s'effondrent à la deuxième page lorsque l'anneau $\mathbb{k}$ est de dimension au plus 1 et procurent alors des isomorphismes non fonctoriels

$$
H_{n}\left(O_{\infty}(k) ; F_{\infty}\right) \simeq \bigoplus_{p+q=n} H_{p}\left(O_{\infty}(k) ; \operatorname{Tor}_{q}^{\mathcal{E}^{f}(k)}\left(\mathbb{k}\left[S^{2}\right]^{\vee}, F\right)\right)
$$

et

$$
H_{n}\left(\operatorname{Sp}_{\infty}(k) ; F_{\infty}\right) \simeq \bigoplus_{p+q=n} H_{p}\left(\operatorname{Sp}_{\infty}(k) ; \operatorname{Tor}_{q}^{\delta^{f}(k)}\left(\mathbb{k}\left[\Lambda^{2}\right]^{\vee}, F\right)\right)
$$

Avant de démontrer ces résultats, on donne quelques définitions puis un lemme reliant les différents foncteurs introduits ainsi que ceux de l'appendice D. Les mentions au corps $k$ sont sous-entendues.

DÉfinition 3.24. - On désigne par $\mathcal{E}_{\text {iso }}^{f}$ la sous-catégorie de $\mathcal{E}^{f}$ ayant les mêmes objets et les isomorphismes pour morphismes.

Dans ce qui suit, on utilise également les catégories introduites dans la définition D.1.

DÉfinition 3.25. - $\quad-$ Le foncteur $\beta: \operatorname{Mod}-\mathcal{E}_{\text {iso }}^{f} \rightarrow \operatorname{Mod}-\mathcal{E}_{\text {surj }}^{f}$ envoie un foncteur $F$ sur le foncteur prenant les mêmes valeurs sur les objets et les isomorphismes et envoyant les surjections strictes sur 0 .

- Le foncteur $\gamma: \mathbf{M o d}-\delta_{q}^{\text {deg }}\left[(-\oplus \mathbf{H})^{-1}\right] \rightarrow \mathbf{M o d}-\delta_{q}^{\text {deg }}$ est la précomposition par le foncteur canonique $\phi: \mathcal{E}_{q}^{\mathrm{deg}} \rightarrow \mathcal{E}_{q}^{\mathrm{deg}}\left[(-\oplus \mathbf{H})^{-1}\right]$.

- Le foncteur $\delta: \operatorname{Mod}-\delta^{f} \rightarrow \operatorname{Mod}-\mathcal{E}_{\text {inj }}^{f}$ est la précomposition par le foncteur d'inclusion $\mathcal{E}_{\text {inj }}^{f} \rightarrow \mathcal{E}^{f}$.

Remarque 3.26. - On rappelle qu'une forme quadratique sur un espace vectoriel $V$ est un élément de $S^{2}\left(V^{*}\right)$. Par conséquent, pour $k$ un corps commutatif (éventuellement de caractéristique 2$)$, on a l'égalité :

$$
\mathcal{E}_{q}^{\mathrm{deg}}=\left(\mathcal{E}_{\mathrm{inj}}^{f}\right)_{\left(S^{2}\right)^{\vee}}
$$

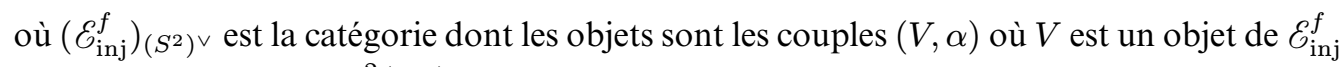
et $\alpha$ est un élément de $S^{2}\left(V^{*}\right)$. On note

$$
\Omega_{\left(S^{2}\right)^{\vee}}: \operatorname{Mod}-\mathcal{E}_{q}^{\mathrm{deg}}=\operatorname{Mod}-\left(\mathcal{E}_{\mathrm{inj}}^{f}\right)_{\left(S^{2}\right)^{\vee}} \rightarrow \operatorname{Mod}-\mathcal{E}_{\mathrm{inj}}^{f}
$$

le foncteur défini à la proposition $\mathrm{A} .3$ de l'appendice $\mathrm{A}$.

La preuve du théorème 3.21 repose sur le lemme suivant, qui combine les résultats $\mathrm{du} \S 3.1$ et la décomposition des foncteurs de Mackey. On rappelle que les foncteurs $\rho$, $\kappa$ et $\omega$ sont introduits dans la définition D.1. 
Lemme 3.27. - Le diagramme suivant :

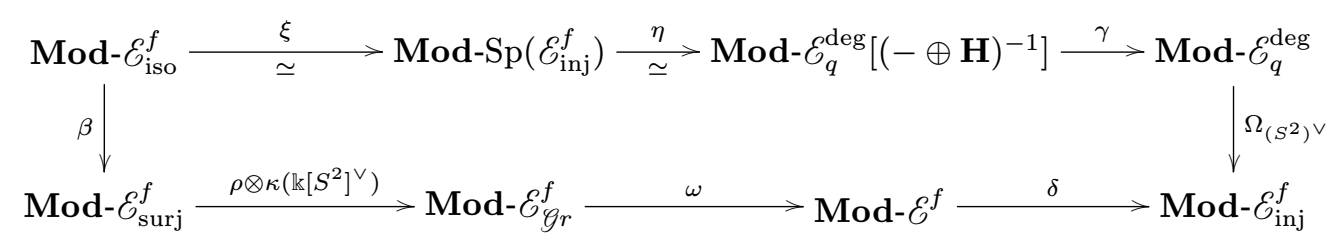

où $\eta$ est induit par l'équivalence de catégories du théorème 3.17 et $\xi$ est l'équivalence donnée dans le théorème C.5, commute à isomorphisme canonique près.

Démonstration. - Soit $\alpha$ un objet de Mod- $\varepsilon_{\text {iso }}^{f}$. Son image dans Mod- $\varepsilon_{\text {inj }}^{f}$ en suivant le chemin supérieur est donnée par

$$
V \mapsto \bigoplus_{q \in S^{2}\left(V^{*}\right)} \bigoplus_{W \subset \operatorname{Rad}(V, q)} \alpha(W)
$$

Sur les morphismes, une flèche $f: V \rightarrow V^{\prime}$ de $\mathcal{E}_{\text {inj }}^{f}$ est transformée en l'application linéaire dont la composante $\alpha\left(W^{\prime}\right) \rightarrow \alpha(W)$, pour $q \in S^{2}\left(V^{*}\right), q^{\prime} \in S^{2}\left(V^{\prime *}\right), W \subset \operatorname{Rad}(V, q)$ et $W^{\prime} \subset \operatorname{Rad}\left(V^{\prime}, q^{\prime}\right)$, est $\alpha(\bar{f})$ lorsque $S^{2}\left({ }^{t} f\right)\left(q^{\prime}\right)=q$ et que $f$ induit un isomorphisme $\bar{f}: W \rightarrow W^{\prime}$ (i.e. $f(W)=W^{\prime}$ ) et est nulle sinon.

On peut simplifier cette écriture en notant que la donnée d'une forme quadratique $q$ sur $V$ et d'un sous-espace $W$ de $V$ inclus dans son radical est équivalente à la donnée d'un sousespace $W$ de $V$ et d'une forme quadratique $\bar{q}$ sur $V / W$. Notre foncteur devient alors

$$
V \mapsto \bigoplus_{W \subset V} \alpha(W) \otimes \mathbb{k}\left[S^{2}\left((V / W)^{*}\right)\right]
$$

Sur les morphismes, la composante $\alpha\left(W^{\prime}\right) \otimes \mathbb{k}\left[S^{2}\left(\left(V^{\prime} / W^{\prime}\right)^{*}\right)\right] \rightarrow \alpha(W) \otimes \mathbb{k}\left[S^{2}\left((V / W)^{*}\right)\right]$ induite par $f$ est nulle si $f(W) \neq W^{\prime}$ et est sinon égale au produit tensoriel de $\alpha(\bar{f})$ et du morphisme $\mathbb{k}\left[S^{2}\left(\left(V^{\prime} / W^{\prime}\right)^{*}\right)\right] \rightarrow \mathbb{k}\left[S^{2}\left((V / W)^{*}\right)\right]$ associant $\left[\overline{S^{2}\left({ }^{t} f\right)\left(q^{\prime}\right)}\right]$ à $\left[\overline{q^{\prime}}\right]$ pour $q^{\prime}$ forme quadratique sur $V^{\prime}$ nulle sur $W^{\prime}$. Ce dernier morphisme n'étant autre que $\kappa\left(\mathbb{k}\left[S^{2}\right]^{\vee}\right)(f)$, cela établit le lemme.

Démonstration du théorème 3.21. - On se contente d'établir l'assertion relative au groupe orthogonal, l'autre étant tout à fait analogue.

On utilise la notation $L_{q}$ de 2.21 , le triplet sous-jacent étant $\left(\mathcal{E}_{q}^{\mathrm{deg}}, S_{\mathbf{H}}, G_{\mathbf{H}}\right)$ (cf. exemple 1.9. 1).

Compte tenu de la proposition A.3, la suite spectrale de la proposition 2.19 prend la forme :

$$
E_{p, q}^{2}=\operatorname{Tor}_{p}^{\delta_{q}^{\mathrm{deg}}}\left(L_{q}, U^{*} F\right) \simeq \operatorname{Tor}_{p}^{\delta_{\mathrm{inj}}^{f}}\left(\Omega_{\left(S^{2}\right)^{\vee}}\left(L_{q}\right), F\right) \Rightarrow H_{p+q}\left(O_{\infty} ; F_{\infty}\right)
$$

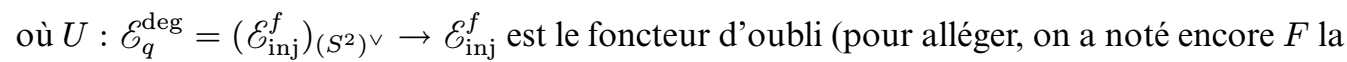
restriction de ce foncteur à $\mathcal{E}_{\mathrm{inj}}^{f}$ ).

La proposition 2.22 montre que les foncteurs $L_{q}$ inversent les inclusions $V \hookrightarrow V \perp H$, où l'espace quadratique $H$ est non dégénéré; ils appartiennent par conséquent à l'image essentielle du foncteur $\gamma$. Il existe donc, par le lemme précédent, des objets $\alpha_{q}$ de Mod- $\mathcal{E}_{\text {iso }}^{f}$ tels que $\Omega_{\left(S^{2}\right)^{\vee}}\left(L_{q}\right) \simeq \delta \omega\left(\rho \beta\left(\alpha_{q}\right) \otimes \kappa\left(\mathbb{k}\left[S^{2}\right]^{\vee}\right)\right)$. Autrement dit, $\Omega_{\left(S^{2}\right)^{\vee}}\left(L_{q}\right)$ est isomorphe à la restriction à $\mathcal{E}_{\text {inj }}^{f}$ du foncteur $\omega\left(\rho \beta\left(\alpha_{q}\right) \otimes \kappa\left(\mathbb{k}\left[S^{2}\right]^{\vee}\right)\right)$ de Mod- $\mathcal{E}^{f}$. 
Comme $F$ est par hypothèse analytique, on déduit du théorème D.5 (dû à Suslin) que le morphisme canonique $\operatorname{Tor}_{p}^{\delta_{\text {inj }}^{f}}\left(\Omega_{\left(S^{2}\right)^{\vee}}\left(L_{q}\right), F\right) \rightarrow \operatorname{Tor}_{p}^{\mathcal{E}^{f}}\left(\omega\left(\rho \beta\left(\alpha_{q}\right) \otimes \kappa\left(\mathbb{k}\left[S^{2}\right]^{\vee}\right)\right), F\right)$ est un isomorphisme.

Le caractère analytique de $F$ implique également que le monomorphisme canonique $\lambda\left(\rho \beta\left(\alpha_{q}\right) \otimes \kappa\left(\mathbb{k}\left[S^{2}\right]^{\vee}\right)\right) \rightarrow \omega\left(\rho \beta\left(\alpha_{q}\right) \otimes \kappa\left(\mathbb{k}\left[S^{2}\right]^{\vee}\right)\right)$ (le foncteur $\lambda$ est défini en D.1), induit un isomorphisme $\operatorname{Tor}_{p}^{\delta^{f}}\left(\lambda\left(\rho \beta\left(\alpha_{q}\right) \otimes \kappa\left(\mathbb{k}\left[S^{2}\right]^{\vee}\right)\right), F\right) \rightarrow \operatorname{Tor}_{p}^{\delta^{f}}\left(\omega\left(\rho \beta\left(\alpha_{q}\right) \otimes \kappa\left(\mathbb{k}\left[S^{2}\right]^{\vee}\right)\right), F\right)$, grâce au théorème D.2.

On note à présent que $\lambda\left(\rho \beta\left(\alpha_{q}\right) \otimes \kappa\left(\mathbb{k}\left[S^{2}\right]^{\vee}\right)\right)$ est isomorphe à $\alpha_{q}(0) \otimes \mathbb{k}\left[S^{2}\right]^{\vee}$. Quant au $\mathbb{k}$-module $\alpha_{q}(0)$, c'est la valeur en 0 du foncteur $L_{q}$, c'est-à-dire $H_{q}\left(O_{\infty}(k) ; \mathbb{k}\right)$.

Par la proposition 2.25, on en déduit que le morphisme naturel $H_{*}\left(O_{\infty}(k) ; F_{\infty}\right) \rightarrow H_{*}\left(\mathcal{E}_{q}^{\mathrm{deg}}(k) \times O_{\infty}(k) ; U^{*} F\right)$ est un isomorphisme. La proposition A.3 fournit par ailleurs un isomorphisme entre $H_{*}\left(\mathcal{E}_{q}^{\mathrm{deg}}(k) \times O_{\infty}(k) ; U^{*} F\right)$ et $\operatorname{Tor}_{*}^{\varepsilon_{\text {inj }}^{f} \times O_{\infty}(k)}\left(\mathbb{k}\left[S^{2}\right]^{\vee}, F\right)$, lui-même isomorphe à $\operatorname{Tor}_{*}^{\mathcal{E}^{f} \times O_{\infty}(k)}\left(\mathbb{k}\left[S^{2}\right]^{\vee}, F\right)$ grâce au théorème de Suslin (D.5) déjà évoqué (comparer les suites spectrales de Künneth associées à ces deux derniers groupes de torsion). Cela termine la démonstration.

Démonstration des corollaires 3.22 et 3.23. - Ils se déduisent du théorème 3.21 et des propositions 2.26 et 2.27 respectivement, en utilisant comme précédemment la proposition A. 3 et le théorème D. 5 pour l'identification des deuxièmes pages.

Remarque 3.28. - On vient de montrer que le morphisme naturel de $\operatorname{Tor}_{p}^{\varepsilon_{q}}(M, F)$ dans $\operatorname{Tor}_{p}^{\varepsilon_{q}^{\operatorname{deg}}}(M, F)$, où $M$ est un foncteur constant et $F$ un foncteur analytique de $\mathcal{F}(k)$ (par abus on a omis la notation de précomposition par différents foncteurs d'oubli), est un isomorphisme.

Remarque 3.29. - L'hypothèse de finitude du corps $k$ ne sert essentiellement que dans le lemme 3.27, pour pouvoir affirmer que le foncteur $\xi$ du théorème C.5 est une équivalence de catégories (l'application dudit théorème à la catégorie $\mathcal{E}_{\text {inj }}^{f}$ suppose en effet que l'ensemble des sous-espaces d'un $k$-espace vectoriel de dimension finie soit fini).

Nous pensons cependant que le théorème 3.21 reste valable lorsque $k$ est un corps commutatif infini.

Notation 3.30. - Dans la catégorie $\mathcal{F}(k)$, on désigne par $I$ le foncteur $\left(\mathbb{k}[-]^{\vee}\right)^{*}: V \mapsto \mathbb{k}^{V^{*}}$.

(C'est un objet injectif de $\mathcal{F}$.)

En utilisant la trivialité de l'homologie stable des groupes classiques sur un corps fini à coefficients dans le même corps (voir [12], chapitre 3 , §4), la caractéristique 2 étant exclue pour les groupes orthogonaux, on déduit du théorème 3.21 le résultat suivant. Dans le cas où $k$ est de caractéristique 2 , l'homologie $H_{*}\left(O_{\infty}(k) ; \mathbb{F}_{2}\right)$ n'est pas triviale mais isomorphe à $H_{*}\left(\mathbb{Z} / 2 ; \mathbb{F}_{2}\right)$ (donc de dimension 1 en chaque degré), car le sous-groupe d'indice 2 de $O_{\infty}(k)$, noté $D O$ dans [12] (défini au chapitre 2, §7, via l'invariant de Dickson), analogue en caractéristique 2 du groupe spécial orthogonal, a une homologie triviale (cf. [12], chapitre $3, \S 4)$.

$4^{\mathrm{e}}$ SÉRIE - TOME $43-2010-\mathrm{N}^{\mathrm{o}} 3$ 
Corollaire 3.31. - Supposons que $\mathbb{k}=k$ est un corps fini de caractéristique $p$. Si F est un foncteur analytique de $\mathcal{F}(k)$, il existe des isomorphismes naturels

$$
\begin{aligned}
H_{*}\left(O_{\infty}(k) ; F_{\infty}\right) & \simeq \operatorname{Tor}_{*}^{\varepsilon^{f}}\left(k\left[S^{2}\right]^{\vee}, F\right) \text { si p est impair, } \\
H_{*}\left(O_{\infty}(k) ; F_{\infty}\right) & \simeq \operatorname{Tor}_{*}^{\mathcal{E}^{f}}\left(k\left[S^{2}\right]^{\vee}, F\right) \otimes H_{*}(\mathbb{Z} / 2 ; k) \text { si } p=2 \\
\text { et } \quad H_{*}\left(\operatorname{Sp}_{\infty}(k) ; F_{\infty}\right) & \simeq \operatorname{Tor}_{*}^{\delta^{f}}\left(k\left[\Lambda^{2}\right]^{\vee}, F\right) .
\end{aligned}
$$

Les duaux de ces espaces vectoriels s'identifient canoniquement à

$$
\begin{array}{ll} 
& \operatorname{Ext}_{\mathscr{\mathcal { G }}}^{*}\left(F, I \circ \Gamma^{2}\right) \quad \text { si p est impair, } \\
& \operatorname{Ext}_{\mathscr{\mathcal { Y }}}^{*}\left(F, I \circ \Gamma^{2}\right) \otimes H^{*}(\mathbb{Z} / 2 ; k) \quad \text { si } p=2 \\
\text { et } \quad \operatorname{Ext}_{\mathscr{J}}^{*}\left(F, I \circ \Lambda^{2}\right) \quad \text { respectivement. }
\end{array}
$$

En utilisant les résultats de stabilité établis par Charney dans [8], on en déduit le corollaire suivant.

Corollaire 3.32. - Sous les hypothèses précédentes, supposons de plus que F est polynomial de degré au plus $d$. Alors pour tous entiers $i$ et $n$ tels que $n \geq 2 i+d+6$, on a des isomorphismes naturels

$$
\begin{aligned}
H_{i}\left(O_{n, n}(k) ; F\left(k^{2 n}\right)\right)^{*} & \simeq \operatorname{Ext}_{\mathscr{F}}^{i}\left(F, I \circ \Gamma^{2}\right) \quad \text { si p est impair, } \\
H_{i}\left(O_{n, n}(k) ; F\left(k^{2 n}\right)\right)^{*} & \simeq \bigoplus_{j=0}^{i} \operatorname{Ext}_{\mathscr{F}}^{j}\left(F, I \circ \Gamma^{2}\right) \text { si } p=2 \\
\text { et } \quad H_{i}\left(\operatorname{Sp}_{2 n}(k) ; F\left(k^{2 n}\right)\right)^{*} & \simeq \operatorname{Ext}_{\mathscr{G}}^{i}\left(F, I \circ \Lambda^{2}\right) .
\end{aligned}
$$

Par dualité entre homologie et cohomologie d'un groupe fini, on obtient la variante suivante :

COROLlaire 3.33. - Sous les mêmes hypothèses, il existe des isomorphismes naturels

$$
\begin{aligned}
H^{i}\left(O_{n, n}(k) ; F\left(k^{2 n}\right)\right) & \simeq \operatorname{Ext}_{\mathscr{G}}^{i}\left(k\left[S^{2}\right], F\right) \quad \text { si } p \text { est impair } \\
H^{i}\left(O_{n, n}(k) ; F\left(k^{2 n}\right)\right) & \simeq \bigoplus_{j=0}^{i} \operatorname{Ext}_{\mathscr{G}}^{i}\left(k\left[S^{2}\right], F\right) \quad \text { si } p=2 \\
\text { et } \quad H^{i}\left(\operatorname{Sp}_{2 n}(k) ; F\left(k^{2 n}\right)\right) & \simeq \operatorname{Ext}_{\mathscr{G}}^{i}\left(k\left[\Lambda^{2}\right], F\right) .
\end{aligned}
$$

\section{Quelques calculs d'homologie de groupes orthogonaux et symplectiques}

Nous allons maintenant illustrer les résultats de la section précédente par des calculs explicites de groupes d'homologie de groupes orthogonaux et symplectiques.

Convention 4.1. - Dans toute cette section, on suppose que $\mathbb{k}=k$ est un corps fini de caractéristique $p$ et de cardinal $q=p^{d}$.

On rappelle qu'on note simplement $\mathcal{F}(k)$ ou $\mathcal{F}$ la catégorie de foncteurs $\mathcal{E}^{f}(k)$-Mod.

Les mentions au corps $k$ seront souvent omises. 
On s'est limité au cas $k=\mathbb{k}$ car tout foncteur polynomial sans terme constant de $\mathcal{E}_{k}^{f}$ vers les groupes abéliens prend ses valeurs dans les $\mathbb{F}_{p}$-espaces vectoriels; une extension des scalaires au but ne modifie guère le comportement homologique de notre catégorie de foncteurs. De surcroît, c'est le cas où $\mathbb{k}$ égale $k$ qu'il est usuel d'étudier; des calculs d'algèbre homologique poussés y ont été effectués (cf. [15] et [14]).

\subsection{Compatibilité aux (co)produits}

Si $G$ est un groupe et $M$ et $N$ sont deux $G$-modules, la projection canonique $(M \otimes N)_{G} \rightarrow M_{G} \otimes N_{G}$ induit un coproduit externe en homologie $H_{*}(G ; M \otimes N) \rightarrow$ $H_{*}(G ; M) \otimes H_{*}(G ; N)$. Ce morphisme naturel gradué fait de $H^{*}(G ;-)$ un foncteur comonoïdal. Il est plus usuel de considérer la situation duale, à savoir le produit externe

$$
H^{*}(G ; M) \otimes H^{*}(G ; N) \rightarrow H^{*}(G ; M \otimes N)
$$

qui fait de $H^{*}(G ;-)$ un foncteur monoïdal des $G$-modules vers les espaces vectoriels gradués et se réduit en degré 0 à l'inclusion canonique $M^{G} \otimes N^{G} \hookrightarrow(M \otimes N)^{G}$.

Des constructions analogues existent en (co)homologie des foncteurs : le produit tensoriel induit en particulier, pour toute petite catégorie $\mathscr{C}$, des produits naturels

$$
\operatorname{Ext}_{\mathscr{C}}^{*}(A, F) \otimes \operatorname{Ext}_{\mathscr{C}}^{*}(B, G) \rightarrow \operatorname{Ext}_{\mathscr{C}}^{*}(A \otimes B, F \otimes G) .
$$

(On a des morphismes duaux évidents dans le cas des groupes de torsion.)

Lorsque le foncteur $A$ est muni d'une structure de cogèbre, on peut utiliser les morphismes $\operatorname{Ext}^{*}(A \otimes A, F \otimes G) \rightarrow \operatorname{Ext}^{*}(A, F \otimes G)$ induits par le coproduit $A \rightarrow A \otimes A$ pour en déduire un produit

$$
\operatorname{Ext}^{*}(A, F) \otimes \operatorname{Ext}^{*}(A, G) \rightarrow \operatorname{Ext}^{*}(A, F \otimes G) .
$$

Tous les foncteurs de précomposition, donc en particulier d'évaluation, sont compatibles aux (co)produits ainsi définis. Cela permet d'obtenir la compatibilité de tous les isomorphismes de la section précédente aux produits ou coproduits. Nous nous bornerons ici à l'énoncé suivant :

Proposition 4.2. - Les isomorphismes du corollaire 3.33 sont compatibles aux produits externes. Plus précisément, soient $F$ et $G$ des foncteurs polynomiaux de $\mathcal{F}$ de degrés respectifs $d$ et $d^{\prime}$ et $n, i, j$ des entiers tels que $n \geq 2(i+j)+d+d^{\prime}+6$. Supposons la caractéristique $p$ de $k$ impaire. Le diagramme

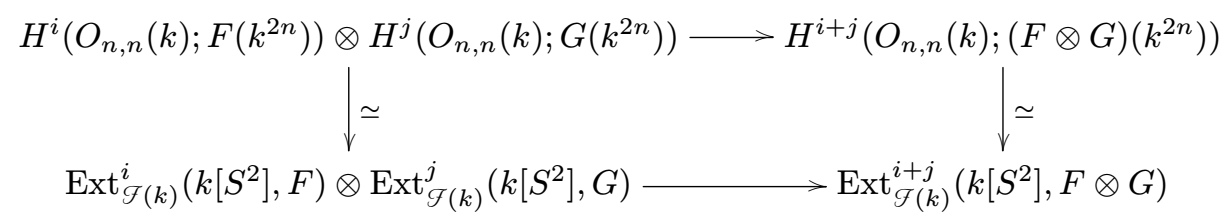

dans lequel les flèches horizontales sont les produits et les flèches verticales les isomorphismes donnés par le corollaire 3.33 commute. On dispose d'un énoncé analogue évident en termes de groupes symplectiques.

$4^{\text {e }}$ SÉRIE - TOME $43-2010-\mathrm{N}^{\mathrm{o}} 3$ 
Démonstration. - Compte tenu de la remarque précédente et de la description des isomorphismes, il suffit de démontrer que l'isomorphisme d'adjonction $\operatorname{Tor}_{*}^{\varepsilon_{\text {inj }}^{f}}\left(k\left[S^{2}\right]^{\vee}, F\right) \simeq H_{*}\left(\mathcal{E}_{q}^{\mathrm{deg}} ; F\right)$ est compatible aux coproduits (notre énoncé s'en déduit en dualisant). Celui-ci est composé du morphisme $H_{*}\left(\delta_{q}^{\mathrm{deg}} ; F\right)=$ $\operatorname{Tor}_{*}^{\varepsilon_{*}^{\mathrm{deg}}}(k ; F) \rightarrow \operatorname{Tor}_{*}^{\delta_{q}^{\mathrm{deg}}}\left(k\left[S^{2}\right]^{\vee} ; F\right)$ induit par la flèche $k \rightarrow k\left[S^{2}\right]^{\vee}$ de Mod- $\delta_{q}^{\text {deg }}$ donnée, sur un espace quadratique $(V, q)$, par l'élément $[q]$ de $k\left[S^{2}\left(V^{*}\right)\right]$, et du morphisme $\operatorname{Tor}_{*}^{\delta_{q}^{\text {deg }}}\left(k\left[S^{2}\right]^{\vee} ; F\right) \rightarrow \operatorname{Tor}_{*}^{\varepsilon_{\text {inj }}^{f}}\left(k\left[S^{2}\right]^{\vee} ; F\right)$ induit par le foncteur d'oubli de la forme quadratique $\mathcal{E}_{q}^{\mathrm{deg}} \rightarrow \mathcal{E}_{\mathrm{inj}}^{f}$. Le premier respecte les coproduits car $k \rightarrow k\left[S^{2}\right]^{\vee}$ est un morphisme de cogèbres, le second par l'observation générale précédant la démonstration, d'où la proposition.

Cette proposition permet d'obtenir une propriété générale frappante des produits externes en cohomologie stable des groupes orthogonaux, à l'aide d'observations élémentaires mais efficaces dues à Touzé - la proposition suivante est établie (dans le contexte analogue des foncteurs polynomiaux stricts) dans [30].

Proposition 4.3 (Touzé). - Soient $\mathscr{G}$ une petite catégorie additive et $A$ un objet de $\mathscr{G}$-Mod muni d'une structure de cogèbre et d'un épimorphisme naturellement scindé $A(V \oplus W) \rightarrow A(V) \otimes A(W)$ pour $V, W \in \mathrm{Ob} \mathscr{C}$ de sorte que le coproduit de $A$ soit donné par la composition $A(V) \rightarrow A(V \oplus V) \rightarrow A(V) \otimes A(V)$, où la première flèche est induite par la diagonale $V \rightarrow V \oplus V$. On suppose de surcroît soit que A possède une résolution projective de type fini, soit que $F$ et $G$ possèdent une résolution injective de type fini et que $A$ prend des valeurs de dimension finie. Alors le produit externe

$$
\operatorname{Ext}^{*}(A, F) \otimes \operatorname{Ext}^{*}(A, G) \rightarrow \operatorname{Ext}^{*}(A, F \otimes G)
$$

est une injection naturellement scindée pour tous objets $F$ et $G$ de $\mathscr{Q}$-Mod.

Démonstration. - À l'aide de l'épimorphisme scindé de l'hypothèse, des foncteurs adjoints $\oplus: \mathscr{G} \times \mathscr{G} \rightarrow \mathscr{G}$ et $\delta: \mathscr{G} \rightarrow \mathscr{G} \times \mathscr{G}$ (diagonale) et de la formule de Künneth (cf. par exemple [14], propriété (1.7.2) ${ }^{(1)}$ ), on obtient un monomorphisme naturellement scindé

$$
\begin{aligned}
& \operatorname{Ext}_{\mathscr{Q}}^{*}(A, F) \otimes \operatorname{Ext}_{\mathscr{G}}^{*}(A, G) \simeq \operatorname{Ext}_{\mathscr{Q} \times \mathscr{Q}}^{*}(A \otimes A, F \otimes G) \hookrightarrow \operatorname{Ext}_{\mathscr{Q} \times \mathscr{Q}}^{*}\left(\oplus^{*} A, F \otimes G\right) \\
& \simeq \operatorname{Ext}_{\mathscr{G}}^{*}\left(A, \delta^{*}(F \otimes G)\right)=\operatorname{Ext}_{\mathscr{Q}}^{*}(A, F \otimes G) .
\end{aligned}
$$

La description du coproduit sur $A$ à partir de l'épimorphisme scindé $\oplus^{*} A \rightarrow A \otimes A$ contenue dans l'hypothèse montre que la flèche précédente n'est autre que le produit de l'énoncé.

On rappelle au lecteur que les généralités concernant les foncteurs exponentiels, dont on fait un usage fréquent dans la suite, sont données dans l'appendice $\mathrm{B}$.

L'hypothèse sur le foncteur $A$ est en particulier vérifiée, pour $\mathscr{C}=\mathcal{E}^{f}$, lorsque $A$ est la composition $E \circ T$ d'un foncteur exponentiel $E$ et d'un foncteur $T$ tel que $T(0)=0$ (puisqu'alors $T(U \oplus V)$ contient $T(U) \oplus T(V)$ comme facteur direct naturel). Par conséquent, les propositions 4.2 et 4.3 procurent le résultat d'injectivité suivant (où l'on utilise que les

(1) Dans cet énoncé, il n'est fait mention de la formule de Künneth que dans le premier cas de finitude envisagé; l'autre se traite de façon analogue. 
foncteurs polynomiaux à valeurs de dimension finie possèdent des résolutions injectives de type fini — cf. [15], §10, où l'énoncé est donné sous forme duale).

Proposition 4.4. - Soient $F$ et $G$ deux foncteurs polynomiaux de $\mathcal{F}(k)$ prenant des valeurs de dimension finie, de degrés respectifs $d$ et $d^{\prime}, i, j$ et $n$ des entiers tels que $n \geq 2(i+j)+d+d^{\prime}+6$. Alors le produit externe

$$
H^{i}\left(O_{n, n}(k) ; F\left(k^{2 n}\right)\right) \otimes H^{j}\left(O_{n, n}(k) ; G\left(k^{2 n}\right)\right) \rightarrow H^{i+j}\left(O_{n, n}(k) ;(F \otimes G)\left(k^{2 n}\right)\right)
$$

est injectif si la caractéristique de $k$ est impaire.

Remarque 4.5. - On a en fait mieux (cf. [30], §6.1) : il existe en cohomologie stable des groupes orthogonaux ou symplectiques un coproduit externe qui fournit une rétraction naturelle au produit externe, comme on s'en convainc aisément en reprenant la démonstration. Il est impossible de décrire directement ce coproduit, qui n'existe pas en cohomologie instable.

Remarque 4.6. - Il est en fait inutile d'invoquer les propriétés de finitude des foncteurs polynomiaux à valeurs de dimension finie. En effet, la variante duale (en termes de groupes de torsion) de la proposition 4.3 est valide sans aucune hypothèse de finitude (comme la formule de Künneth pour les Tor); les groupes d'extensions ici considérés viennent tous de la dualisation de groupes de torsion. Nous avons néanmoins privilégié les énoncés en termes de groupes d'extensions, plus usuels et plus intuitifs.

Nous donnons maintenant quelques résultats préliminaires aux calculs explicites de (co)homologie stabilisée de groupes orthogonaux et symplectiques tordus par des foncteurs polynomiaux classiques. Nos calculs concerneront les foncteurs exponentiels gradués usuels : puissances extérieures, divisées, symétriques.

Lemme 4.7. - Soit F un objet de $\mathcal{F}$. Il existe un isomorphisme gradué naturel

$$
\operatorname{Tor}_{*}^{\mathcal{E}^{f}}\left(k\left[T^{2}\right]^{\vee}, F\right) \simeq H H_{*}\left(\mathcal{E}^{f} ;(V, W) \mapsto F\left(V^{*} \oplus W\right)\right) .
$$

Démonstration. - Utilisant l'adjonction entre les foncteurs exacts induits par la somme directe et la diagonale, l'équivalence de catégories $(-)^{*}:\left(\mathcal{E}^{f}\right)^{\mathrm{op}} \rightarrow \mathcal{E}^{f}$ et l'isomorphisme naturel $V^{*} \otimes W \simeq \operatorname{Hom}_{\mathcal{E}^{f}}(V, W)$, puis l'isomorphisme (10) de l'appendice A, on obtient des isomorphismes gradués naturels

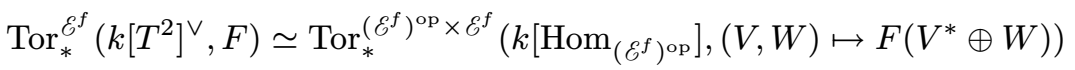

$$
\begin{aligned}
& \simeq H H_{*}\left(\mathcal{E}^{f} ;(V, W) \mapsto F\left(V^{*} \oplus W\right)\right) .
\end{aligned}
$$

Remarque 4.8. - Pour un foncteur polynomial $F$, par le théorème de Betley-Suslin [4] [14, appendice], on a un isomorphisme naturel

$$
H H_{*}\left(\mathcal{E}^{f} ;(V, W) \mapsto F\left(V^{*} \oplus W\right)\right) \simeq \operatorname{colim}_{n \in \mathbb{N}} H_{*}\left(G L_{n}(k) ; F\left(k^{n} \oplus k^{n}\right)\right)
$$

où $g \in G L_{n}(k)$ agit sur $k^{n} \oplus k^{n} \operatorname{par}\left({ }^{t} g^{-1}, g\right)$.

$4{ }^{\mathrm{e}}$ SÉRIE - TOME $43-2010-\mathrm{N}^{\mathrm{o}} 3$ 
On vérifie que le diagramme suivant commute

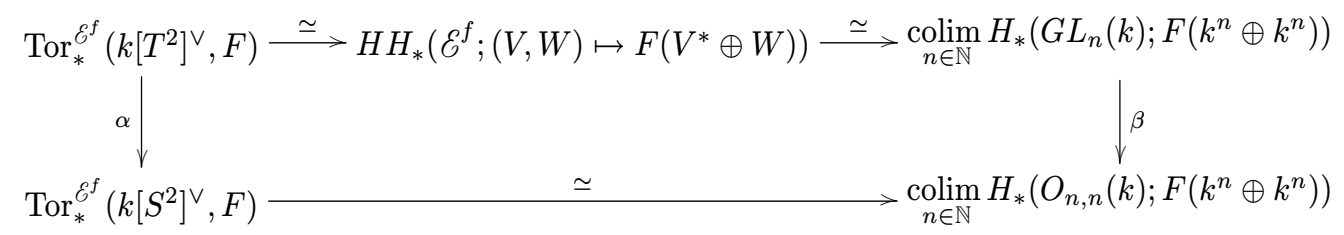

où $\alpha$ est induit par le morphisme canonique $T^{2} \rightarrow S^{2}, \beta$ par le morphisme de groupes

$$
G L_{n}(k) \rightarrow O_{n, n}(k) \quad g \mapsto\left(\begin{array}{cc}
{ }^{t} g^{-1} & 0 \\
0 & g
\end{array}\right)
$$

et l'isomorphisme du bas est induit par le corollaire 3.31.

On note $D: \mathscr{F}^{\text {op }} \rightarrow \mathcal{F}$ le foncteur de dualité de $\mathcal{F}$ composé commutatif de $(-)^{\vee}$ et $(-)^{*}$ (cf. notation 3.19), c'est-à-dire donné par $(D F)(V)=F\left(V^{*}\right)^{*}$. On remarque que ce foncteur vérifie la propriété d'auto-adjonction $\operatorname{Hom}_{\mathscr{F}}(F, D G) \simeq \operatorname{Hom}_{\mathscr{F}}(G, D F)$, qui s'étend aux groupes d'extensions par exactitude de $D$.

Proposition 4.9. - Soit $E^{\bullet}$ un foncteur exponentiel gradué de $\mathcal{F}$. Pour tout entier $n$, il existe un isomorphisme gradué naturel

$$
\operatorname{Tor}_{*}^{\mathcal{E}^{f}}\left(k\left[T^{2}\right]^{\vee}, E^{n}\right) \simeq \bigoplus_{i+j=n} \operatorname{Tor}_{*}^{\mathcal{E}^{f}}\left(\left(E^{i}\right)^{\vee}, E^{j}\right) .
$$

Le dual de cet espace vectoriel s'identifie à

$$
\operatorname{Ext}_{\mathscr{G}}^{*}\left(E^{n}, I \circ T^{2}\right) \simeq \bigoplus_{i+j=n} \operatorname{Ext}_{\mathscr{G}}^{*}\left(E^{j}, D E^{i}\right)
$$

Démonstration. - Le premier isomorphisme s'obtient par le lemme 4.7 en développant $E^{n}\left(V^{*} \oplus W\right)$ à l'aide de la propriété exponentielle et en utilisant l'expression de l'homologie de Hochschild d'un produit tensoriel extérieur comme groupe de torsion.

La deuxième assertion s'obtient à partir de la première et de l'isomorphisme (7) de l'appendice A.

RemARQue 4.10. - Le substitut de propriété exponentielle indiqué dans la remarque B.2 permet d'obtenir d'une manière analogue, pour les puissances tensorielles, un isomorphisme gradué

$$
\operatorname{Tor}_{*}^{\delta^{f}}\left(k\left[T^{2}\right]^{\vee}, T^{n}\right) \simeq \bigoplus_{i+j=n} \operatorname{Tor}_{*}^{\delta^{f}}\left(\left(T^{i}\right)^{\vee}, T^{j}\right) \underset{\mathfrak{S}_{i} \times \mathfrak{S}_{j}}{\otimes} k\left[\mathfrak{S}_{n}\right]
$$

Ces groupes peuvent être entièrement calculés; on laisse au lecteur le soin d'écrire les analogues des résultats de la suite de cette section en termes de puissances tensorielles.

Par la suite, nous utiliserons systématiquement la forme duale en termes d'Ext, car tous les calculs effectués dans la catégorie $\mathcal{F}$ ont été donnés en termes de groupes d'extensions et non de torsion (cf. [13] par exemple).

Dans l'énoncé suivant, les produits et coproduits sont internes; ils s'obtiennent à partir des structures externes en utilisant la structure de foncteur de Hopf. 
Proposition 4.11. - Soit $E^{\bullet}$ un foncteur exponentiel de Hopf commutatif ou anticommutatif de $\mathcal{F}$. L'isomorphisme bigradué

$$
\operatorname{Ext}_{\mathscr{F}}^{*}\left(E^{\bullet}, I \circ T^{2}\right) \simeq \operatorname{Ext}_{\mathscr{F}}^{*}\left(E^{\bullet}, D E^{\bullet}\right)
$$

de la proposition 4.9 est un isomorphisme d'algèbres de Hopf.

Démonstration. - La compatibilité aux unités et co-unités est évidente.

L'isomorphisme

$$
\gamma_{E^{\bullet}}: \operatorname{Ext}_{\mathscr{F}}^{*}\left(E^{\bullet}, I \circ T^{2}\right) \simeq \operatorname{Ext}_{\mathscr{F}}^{*}\left(E^{\bullet}, D E^{\bullet}\right)
$$

de la proposition 4.9 est naturel en $E^{\bullet}$, la naturalité étant relative aux morphismes respectant la structure exponentielle. Nous obtenons la compatibilité aux produits et coproduits en montrant que $\gamma_{E}$ e est monoïdal. (On rappelle qu'un foncteur monoïdal $F$ entre deux catégories monoïdales $\left(\mathscr{G}, \otimes_{A}\right)$ et $\left(\mathscr{B}, \otimes_{B}\right)$ est un foncteur muni de morphismes naturels $F(A) \otimes_{\mathcal{B}} F\left(A^{\prime}\right) \rightarrow F\left(A \otimes_{\mathscr{G}} A^{\prime}\right)$; une transformation naturelle entre foncteurs monoïdaux est monoïdale si elle est compatible à ces morphismes naturels en un sens évident.) En effet, comme le foncteur exponentiel gradué $E^{\bullet}$ est supposé (anti)commutatif, les morphismes de multiplication $E^{\bullet} \otimes E^{\bullet} \rightarrow E^{\bullet}$ et de comultiplication $E^{\bullet} \rightarrow E^{\bullet} \otimes E^{\bullet}$ sont des morphismes de foncteurs exponentiels, ce qui permet de déduire du caractère monoïdal de $\gamma_{E} \bullet$ sa compatibilité aux produits et coproduits en revenant à leur définition.

La démonstration de la proposition 4.9 montre que $\gamma_{E}$ • s'obtient par composition des trois isomorphismes suivants. Pour alléger, on note dans la suite de la démonstration $\sigma: \mathscr{E}^{f} \times \mathcal{E}^{f} \rightarrow \mathcal{E}^{f}$ le foncteur somme directe, $\pi: \mathcal{E}^{f} \times \mathcal{E}^{f} \rightarrow \mathcal{E}^{f}$ le foncteur produit tensoriel, $\delta: \mathscr{E}^{f} \rightarrow \mathcal{E}^{f} \times \mathcal{E}^{f}$ le foncteur diagonal et $b i-\mathcal{F}$ pour $\mathcal{E}^{f} \times \mathcal{E}^{f}$-Mod.

1. l'isomorphisme

$$
\operatorname{Ext}_{b i-\mathcal{G}}\left(\sigma^{*} F, \pi^{*} I\right) \stackrel{\simeq}{\rightarrow} \operatorname{Ext}_{\mathcal{F}}\left(F, I \circ T^{2}\right)
$$

naturel en $F \in \mathrm{Ob} \mathcal{F}$ déduit de l'adjonction entre $\sigma$ et $\delta$. C'est un isomorphisme monoïdal (où la structure monoïdale des deux foncteurs $\mathcal{F}^{\mathrm{op}} \rightarrow \mathscr{E}$ considérés se déduit de la structure d'algèbre sur le foncteur $I$ ) car il est composé du morphisme naturel monoïdal $\operatorname{Ext}_{b i-\mathcal{G}}\left(\sigma^{*} F, \pi^{*} I\right) \rightarrow \operatorname{Ext}_{\mathcal{G}}\left((\sigma \delta)^{*} F, I \circ T^{2}\right)$ induit par le foncteur $\delta$ et du morphisme naturel monoïdal $\operatorname{Ext}_{\mathscr{F}}\left((\sigma \delta)^{*} F, I \circ T^{2}\right) \rightarrow \operatorname{Ext}_{\mathscr{G}}\left(F, I \circ T^{2}\right)$ induit par l'unité $\operatorname{Id}_{\delta^{f}} \rightarrow \sigma \delta$;

2. l'isomorphisme

$$
\operatorname{Ext}_{b i-\mathscr{F}}\left(\sigma^{*} E^{\bullet}, \pi^{*} I\right) \simeq \operatorname{Ext}_{b i-\mathscr{G}}\left(E^{\bullet} \otimes E^{\bullet}, \pi^{*} I\right)
$$

déduit de la structure exponentielle de $E^{\bullet}$, qui est un morphisme naturel monoïdal de foncteurs contravariants depuis les foncteurs exponentiels vers les espaces vectoriels ;

3. l'isomorphisme

$$
\operatorname{Ext}_{b i-\mathscr{F}}^{*}\left(F \otimes G, \pi^{*} I\right) \stackrel{\simeq}{\longrightarrow} \operatorname{Ext}_{\mathscr{T}}^{*}(F, D G)
$$

naturel en $(F, G) \in \mathrm{Ob} \mathcal{F} \times \mathcal{F}$ est également monoïdal. Il suffit de le montrer en degré cohomologique nul, en lequel il prend la forme explicite suivante : à une transformation naturelle $\left(u_{V, W}: F(V) \otimes G(W) \mapsto I(V \otimes W)\right)_{V, W}$, on associe la transformation 
naturelle donnée par la collection d'applications $F(V) \rightarrow G\left(V^{*}\right)^{*}$ adjointes aux applications

$$
F(V) \otimes G\left(V^{*}\right) \stackrel{u_{V, V^{*}}}{\longrightarrow} I\left(V \otimes V^{*}\right) \simeq k^{\text {End } V} \rightarrow k
$$

où la dernière flèche est l'évaluation en le morphisme identique de $V$. Ce morphisme est donc compatible aux produits, ce qui achève d'établir la proposition.

La structure du foncteur $T^{2}$ dépendant de la parité de la caractéristique $p$ de $k$, nous distinguons le cas $p$ impair du cas $p=2$ dans nos investigations ultérieures. Tandis que nous obtiendrons des calculs complets sur les foncteurs usuels dans le premier cas, nous ne pourrons donner que des résultats très partiels dans le second.

\subsection{Calculs en caractéristique impaire}

Convention 4.12. - Dans tout ce paragraphe, on suppose p impair.

On suppose que $E^{\bullet}$ est un foncteur exponentiel de Hopf gradué commutatif ou anticommutatif.

D'une manière générale, si $u: A \rightarrow B$ est une flèche de $\mathcal{F}$ entre foncteurs sans terme constant (i.e. $A(0)=B(0)=0)$, on note $h(u)=(I \circ u)_{*}: \operatorname{Ext}_{\mathscr{F}}^{*}\left(E^{\bullet}, I \circ A\right) \rightarrow \operatorname{Ext}_{\mathscr{F}}^{*}\left(E^{\bullet}, I \circ B\right)$ le morphisme d'algèbres de Hopf considéré dans la proposition B.6.

Comme 2 est inversible dans $k$, le foncteur $T^{2}$ se scinde en somme des deux foncteurs simples $\Gamma^{2}\left(\simeq S^{2}\right)$ et $\Lambda^{2}$. La propriété exponentielle du foncteur $I$ procure donc un isomorphisme $I \circ T^{2} \simeq\left(I \circ \Gamma^{2}\right) \otimes\left(I \circ \Lambda^{2}\right)$. Comme le foncteur constant en $k$ est facteur direct de $I, I \circ \Gamma^{2}$ et $I \circ \Lambda^{2}$ sont en particulier facteurs directs de $I \circ T^{2}$. Pour mener à bien nos calculs, nous avons besoin d'exprimer précisément l'effet des idempotents correspondant à cette décomposition sur les isomorphismes de la proposition 4.9.

À cette fin, on note $\tau$ l'involution du foncteur $T^{2}$ échangeant les deux facteurs du produit tensoriel, puis $e_{\Gamma}=h\left(\frac{1+\tau}{2}\right)$ et $e_{\Lambda}=h\left(\frac{1-\tau}{2}\right)$ les deux idempotents de $\operatorname{Ext}_{\mathscr{G}}^{*}\left(E^{\bullet}, I \circ T^{2}\right)$ dont les images respectives sont $\operatorname{Ext}_{\mathscr{G}}^{*}\left(E^{\bullet}, I \circ \Gamma^{2}\right)$ et $\operatorname{Ext}_{\mathscr{T}}^{*}\left(E^{\bullet}, I \circ \Lambda^{2}\right)$.

Le résultat suivant permettra, dans les cas usuels, de décrire l'involution $h(\tau)$ en termes explicites :

Lemme 4.13. - Dans l'isomorphisme (5) de la proposition 4.9, l'involution $h(\tau)$ est donnée par les isomorphismes $\operatorname{Ext}_{\mathscr{F}}^{*}\left(E^{j}, D E^{i}\right) \stackrel{\cong}{\longrightarrow} \operatorname{Ext}_{\mathscr{G}}^{*}\left(E^{i}, D E^{j}\right)$ fournis par l'auto-adjonction du foncteur $D$ au signe $\epsilon^{i j}$ près, où $\epsilon$ vaut 1 si $E^{\bullet}$ est commutatif et -1 si $E^{\bullet}$ est anticommutatif.

Démonstration. - Si $F$ est un objet de $\mathcal{F}=\mathcal{E}^{f}$-Mod et $G$ un objet de Mod- $\mathscr{E}^{f}$, le diagramme d'isomorphismes suivant commute

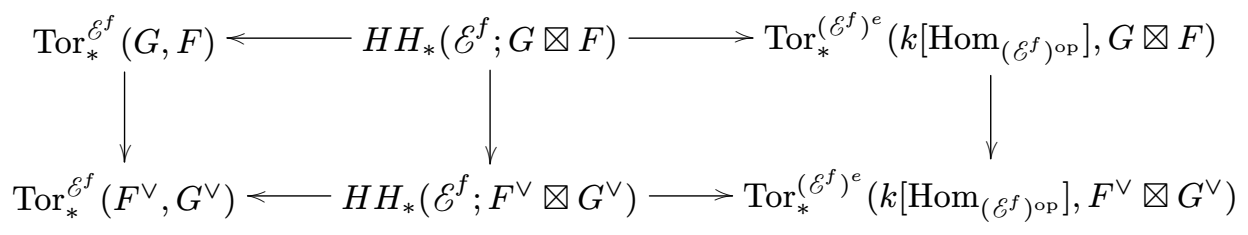

où les flèches verticales sont induites par l'échange des facteurs et l'anti-équivalence de catégories $(-)^{*}$ et où l'on a noté $\left(\mathcal{E}^{f}\right)^{e}=\left(\mathscr{E}^{f}\right)^{\mathrm{op}} \times \mathcal{E}^{f}$. 
Supposons maintenant $F=E^{\bullet}$ et $G=\left(E^{\bullet}\right)^{\vee}$ et formons le diagramme d'isomorphismes suivant

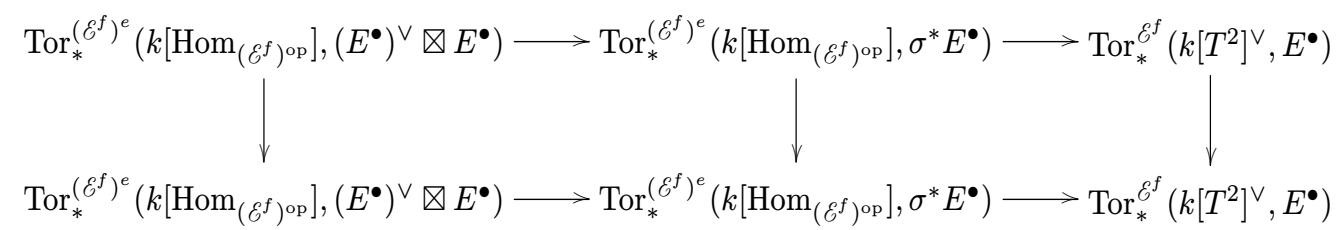

dans lequel les flèches verticales sont construites comme précédemment et où l'on désigne par $\sigma$ le foncteur $\left(\mathcal{E}^{f}\right)^{e} \rightarrow \mathcal{E}^{f}(W, V) \mapsto W^{*} \oplus V$. Le carré de droite commute car les flèches horizontales sont des isomorphismes d'adjonction entre deux foncteurs $(\sigma$ et $\left.V \mapsto\left(V^{*}, V\right)\right)$ invariants par l'auto-équivalence $(W, V) \mapsto\left(V^{*}, W^{*}\right)$ de $\left(\mathcal{E}^{f}\right)^{e}$. Le carré de gauche commute au signe $\epsilon^{i j}$ près lorsque l'on se restreint en degrés covariant $i$ et contravariant $j$, par définition de l'(anti)commutativité de $E^{\bullet}$.

Il suffit alors de reprendre la démonstration de la proposition 4.9 pour obtenir la conclusion.

Le lemme suivant permet de déterminer explicitement, via les résultats de [14], l'involution décrite dans l'énoncé précédent, lorsque $E^{\bullet}$ est un foncteur exponentiel gradué usuel. On y note par abus Id $\in \mathrm{Ob} \mathcal{F}$ le foncteur d'inclusion $\mathcal{E}^{f} \hookrightarrow \mathcal{E}$.

Lemme 4.14. - L'involution sur $\operatorname{Ext}_{\mathcal{G}}^{*}(\mathrm{Id}, \mathrm{Id})$ induite par l'auto-adjonction du foncteur D et l'auto-dualité du foncteur Id est triviale.

Démonstration. - Franjou, Lannes et Schwartz ont déterminé dans l'article [15] $\operatorname{Ext}_{\mathcal{G}}^{*}(\mathrm{Id}, \mathrm{Id})$ : cet espace vectoriel gradué est de dimension 1 en degré pair et nul en degré impair; comme algèbre (pour le produit de composition), $\operatorname{Ext}_{\mathscr{F}}^{*}(\mathrm{Id}, \mathrm{Id})$ est une algèbre symétrique (donc en particulier commutative) sur des générateurs $e_{i}$ de degré $2 p^{i-1}$, pour $i>0$, quotientée par l'idéal des puissances $p$-ièmes. L'involution donnée par la dualité étant un (anti)morphisme d'algèbre graduée, il suffit de vérifier qu'elle préserve les $e_{i}$.

Pour cela, nous aurons besoin d'utiliser la catégorie $\mathscr{P}$ des foncteurs «polynomiaux stricts » sur $k$ de Friedlander-Suslin (cf. [16]). Il existe un foncteur exact $\mathscr{P} \rightarrow \mathcal{F}$ compatible à la dualité ( $\mathscr{P}$ est munie d'une dualité analogue à celle de $\mathscr{F}$ ) tel que $e_{i}$ soit l'image d'un élément, traditionnellement encore noté de la même façon, appartenant à $\operatorname{Ext}_{\mathscr{P}}^{2 p^{i-1}}\left(\operatorname{Id}^{(i)}, \operatorname{Id}^{(i)}\right)$, où $\operatorname{Id}^{(i)}$ désigne le foncteur identité $\mathrm{Id}$ tordu $i$ fois par le morphisme de Frobenius (cf. [16], §4). De plus, le lemme 4.2 de [16] montre que $e_{1}$ est auto-dual. On utilise maintenant le corollaire 5.9 de [14] : il montre que l'image de $e_{i}$ par le morphisme $\operatorname{Ext}_{\mathscr{D}}^{2 p^{i-1}}\left(\operatorname{Id}^{(i)}, \operatorname{Id}^{(i)}\right) \rightarrow \operatorname{Ext}_{\mathscr{P}}^{2 p^{i-1}}\left(\Gamma^{p^{i-1}}(1), S^{p^{i-1}(1)}\right)$ donné par la postcomposition par le morphisme de Frobenius (itéré et tordu) $\mathrm{Id}^{(i)} \rightarrow S^{p^{i-1}(1)}$ et la précomposition par son dual (de sorte que ce morphisme est compatible à la dualité) envoie $e_{i}$ sur $e_{1}^{p^{i-1}}$, où le produit est cette fois relatif à l'algèbre (trigraduée) $\operatorname{Ext}_{\mathscr{P}}^{*}\left(\Gamma^{*(1)}, S^{*(1)}\right)$, dont la structure multiplicative, déduite des structures exponentielles duales de $\Gamma^{*}$ et $S^{*}$, est compatible à la dualité. Il s'ensuit que $e_{i}$ est auto-dual, d'où le lemme. 
Nous rappelons maintenant le premier point du théorème 6.3 de [14], dans la version bigraduée (moins précise que la version trigraduée originelle) qui nous intéresse, où l'assertion relative à la dualité se déduit du lemme 4.14 :

THÉORÈME 4.15 (Franjou-Friedlander-Scorichenko-Suslin). - L'algèbre de Hopf bigraduée $\operatorname{Ext}_{\mathcal{G}}^{*}\left(\Gamma^{\bullet}, S^{\bullet}\right)$ est une algèbre symétrique $S(U)$, où $U$ est un espace vectoriel bigradué de dimension 2 en bidegré $\left(2 q^{s} m, q^{s}+1\right)$ pour tous entiers $m \geq 0$ et $s>0$, de dimension 1 en bidegré $(2 m, 2)$ pour tout entier $m \geq 0$ et nul dans les autres bidegrés. Le premier degré est le degré cohomologique et le second le degré interne.

De surcroît, la dualité est égale à $S(t)$, où t est une involution graduée de $U$ dont 1 est valeur propre simple en chaque bidegré où $U$ est non nul.

On en déduit le théorème suivant :

THÉORÈme 4.16. - L'algèbre de Hopf bigraduée $\operatorname{Ext}_{\mathcal{G}}^{*}\left(\Gamma^{*}, I \circ \Gamma^{2}\right)\left(\right.$ resp. $\left.\operatorname{Ext}_{\mathcal{G}}^{*}\left(\Gamma^{*}, I \circ \Lambda^{2}\right)\right)$ est une algèbre symétrique $S\left(V_{\Gamma}\right)$ (resp. $S\left(W_{\Gamma}\right)$ ), où $V_{\Gamma}$ (resp. $W_{\Gamma}$ ) est un espace vectoriel bigradué de dimension 1 en bidegré $\left(2 q^{s} m, q^{s}+1\right)$ pour tous entiers $m \geq 0$ et $s \geq 0$ (resp. $s>0)$ et nul dans les autres bidegrés.

Démonstration. - Le théorème précédent (dont on conserve les notations) décrit l'algèbre de Hopf bigraduée $\operatorname{Ext}_{\mathcal{F}}^{*}\left(\Gamma^{\bullet}, S^{\bullet}\right) \simeq S(U)$; on a donc aussi $\operatorname{Ext}_{\mathscr{G}}^{*}\left(\Gamma^{\bullet}, I \circ T^{2}\right) \simeq S(U)$ par la proposition 4.11, ainsi que $h(\tau) \simeq S(t)$ par le lemme 4.13. On en déduit par les propositions B.4 et B.6.3

$$
\begin{gathered}
h(1+\tau) \simeq S(1) * S(t) \simeq S(1+t) \quad \text { puis } \\
h\left(\frac{1+\tau}{2}\right)=h\left(\frac{p+1}{2} \cdot(1+\tau)\right)=h(1+\tau)^{* \frac{p+1}{2}} \simeq S(1+t)^{* \frac{p+1}{2}}=S\left(\frac{p+1}{2} \cdot(1+t)\right)=S\left(\frac{1+t}{2}\right) ;
\end{gathered}
$$
de même $h\left(\frac{1-\tau}{2}\right) \simeq S\left(\frac{1-t}{2}\right)$, ce qui donne le résultat.

En procédant de façon similaire, utilisant cette fois la dernière assertion du théorème 6.3 de [14], on obtient le résultat suivant :

THÉORÈme 4.17. - L'algèbre de Hopf bigraduée $\operatorname{Ext}_{\mathcal{F}}^{*}\left(\Lambda^{*}, I \circ \Gamma^{2}\right)\left(\operatorname{resp} . \operatorname{Ext}_{\mathscr{T}}^{*}\left(\Lambda^{*}, I \circ \Lambda^{2}\right)\right)$ est une algèbre à puissances divisées $\Gamma\left(V_{\Lambda}\right)$ (resp. $\Gamma\left(W_{\Lambda}\right)$ ), où $V_{\Lambda}$ (resp. $\left.W_{\Lambda}\right)$ est un espace vectoriel bigradué de dimension 1 en bidegré $\left(2 q^{s} m+q^{s}-1, q^{s}+1\right)$ pour tous entiers $m \geq 0$ et $s>0$ (resp. $s \geq 0$ ) et nul dans les autres bidegrés.

Le corollaire 3.31 et les théorèmes 4.16 et 4.17 impliquent :

THÉORÈME 4.18. - 1. L'algèbre bigraduée

$$
H^{*}\left(O_{\infty} ; S_{\infty}^{\bullet}\right)
$$

est une algèbre symétrique $S\left(V_{S}\right)$, où $V_{S}$ est un espace vectoriel bigradué de dimension 1 en bidegré $\left(2 q^{s} m, q^{s}+1\right)$ pour tous entiers $m \geq 0$ et $s \geq 0$ et nul dans les autres bidegrés.

2. L'algèbre bigraduée

$$
H^{*}\left(O_{\infty} ; \Lambda_{\infty}^{\bullet}\right)
$$

est une algèbre à puissances divisées $\Gamma\left(V_{\Lambda}\right)$, où $V_{\Lambda}$ est un espace vectoriel bigradué de dimension 1 en bidegré $\left(2 q^{s} m+q^{s}-1, q^{s}+1\right)$ pour tous entiers $m \geq 0$ et $s>0$ et nul dans les autres bidegrés. 


\section{L'algèbre bigraduée}

$$
H^{*}\left(\operatorname{Sp}_{\infty} ; S_{\infty}^{\bullet}\right)
$$

est une algèbre symétrique $S\left(W_{S}\right)$, où $W_{S}$ est un espace vectoriel bigradué de dimension 1 en bidegré $\left(2 q^{s} m, q^{s}+1\right)$ pour tous entiers $m \geq 0$ et $s>0$ et nul dans les autres bidegrés.

4. L'algèbre bigraduée

$$
H^{*}\left(\operatorname{Sp}_{\infty} ; \Lambda_{\infty}^{\bullet}\right)
$$

est une algèbre à puissances divisées $\Gamma\left(W_{\Lambda}\right)$, où $W_{\Lambda}$ est un espace vectoriel bigradué de dimension 1 en bidegré $\left(2 q^{s} m+q^{s}-1, q^{s}+1\right)$ pour tous entiers $m \geq 0$ et $s \geq 0$ et nul dans les autres bidegrés.

THÉORÈME 4.19. - $\quad$ 1. La cogèbre bigraduée

$$
H_{*}\left(O_{\infty} ; \Gamma_{\infty}^{\bullet}\right)
$$

est une cogèbre à puissances divisées $\Gamma\left(V_{\Gamma}\right)$, où $V_{\Gamma}$ est un espace vectoriel bigradué de dimension 1 en bidegré $\left(2 q^{s} m, q^{s}+1\right)$ pour tous entiers $m \geq 0$ et $s \geq 0$ et nul dans les autres bidegrés.

2. La cogèbre bigraduée

$$
H_{*}\left(O_{\infty} ; \Lambda_{\infty}^{\bullet}\right)
$$

est une cogèbre symétrique $S\left(V_{\Lambda}\right)$, où $V_{\Lambda}$ est un espace vectoriel bigradué de dimension 1 en bidegré $\left(2 q^{s} m+q^{s}-1, q^{s}+1\right)$ pour tous entiers $m \geq 0$ et $s>0$ et nul dans les autres bidegrés.

3. La cogèbre bigraduée

$$
H_{*}\left(\operatorname{Sp}_{\infty} ; \Gamma_{\infty}^{\bullet}\right)
$$

est une cogèbre à puissances divisées $\Gamma\left(W_{\Gamma}\right)$, où $W_{\Gamma}$ est un espace vectoriel bigradué de dimension 1 en bidegré $\left(2 q^{s} m, q^{s}+1\right)$ pour tous entiers $m \geq 0$ et $s>0$ et nul dans les autres bidegrés.

4. La cogèbre bigraduée

$$
H_{*}\left(\mathrm{Sp}_{\infty} ; \Lambda_{\infty}^{\bullet}\right)
$$

est une cogèbre symétrique $S\left(W_{\Lambda}\right)$, où $W_{\Lambda}$ est un espace vectoriel bigradué de dimension 1 en bidegré $\left(2 q^{s} m+q^{s}-1, q^{s}+1\right)$ pour tous entiers $m \geq 0$ et $s \geq 0$ et nul dans les autres bidegrés.

Par le corollaire 3.32, le théorème précédent calcule les espaces vectoriels $H_{i}\left(O_{n, n}, \Gamma^{j}\left(\mathbb{F}_{q}^{2 n}\right)\right)$, $H_{i}\left(O_{n, n}, \Lambda^{j}\left(\mathbb{F}_{q}^{2 n}\right)\right), H_{i}\left(\mathrm{Sp}_{2 n}, \Gamma^{j}\left(\mathbb{F}_{q}^{2 n}\right)\right)$ et $H_{i}\left(\mathrm{Sp}_{2 n}, \Lambda^{j}\left(\mathbb{F}_{q}^{2 n}\right)\right)$ pour $n \geq 2 i+j+6$.

Pour déterminer par la même méthode $H_{*}\left(O_{\infty}, S_{\infty}^{\bullet}\right)$ et $H_{*}\left(\operatorname{Sp}_{\infty}, S_{\infty}^{\bullet}\right)$, on a besoin de connaître $\operatorname{Ext}_{\mathscr{F}}^{*}\left(S^{\bullet}, \Gamma^{\bullet}\right)$. Ce calcul, manquant dans [14], a été effectué récemment par Chałupnik dans [7], corollaire 4.6 (toujours en transitant par la catégorie $\mathscr{P}$ ). On laisse au lecteur le soin d'écrire le résultat, un petit peu plus technique, obtenu.

Pour conclure ce paragraphe, mentionnons un corollaire frappant de ce théorème, dont les auteurs ignorent s'il peut être établi de manière plus directe.

Corollaire 4.20. - Soient $n$ et $i$ deux entiers tels que $n \geq 2 i+8$. Alors le $i$-ième groupe d'homologie du groupe $O_{n, n}\left(\right.$ resp. $\left.\mathrm{Sp}_{2 n}\right)$ à coefficients dans sa représentation adjointe est nul. 
Démonstration. - Soit $(V, q)$ un espace quadratique de dimension finie non dégénéré. L'isomorphisme d'espaces vectoriels $\varphi: T^{2}(V) \stackrel{\simeq}{\longrightarrow}$ End $V$ composé de l'isomorphisme canonique $T^{2}(V) \simeq \operatorname{Hom}_{k}\left(V^{*}, V\right)$ et de $\operatorname{Hom}_{k}(\phi, V)$, où $\phi: V \stackrel{\simeq}{\longrightarrow} V^{*}$ est l'isomorphisme déduit de $q$, est $O(V, q)$-équivariant, où l'action est la restriction de l'action de $G L(V)$ donnée par la fonctorialité de $T^{2}$ à la source et la conjugaison au but (cf. remarque 2.13). De plus, $\varphi$ se restreint en un isomorphisme $O(V, q)$-équivariant entre $\Lambda^{2}(V)$ et la représentation adjointe de $O(V, q)$.

Par conséquent, l'annulation dans le cas orthogonal provient de celle de $H_{*}\left(O_{\infty} ; \Lambda^{2}\right)$ contenue dans le théorème précédent et du corollaire 3.32 (cf. remarque suivant le théorème précédent).

Le cas symplectique s'obtient de la même manière à partir de l'annulation de $H_{*}\left(\operatorname{Sp}_{\infty} ; S^{2}\right)$.

\subsection{Un calcul en caractéristique 2}

Convention 4.21. - Dans ce paragraphe, $k$ est le corps à 2 éléments $\mathbb{F}_{2}$.

On pourrait procéder de manière analogue sur tout corps fini de caractéristique 2 , mais nous nous restreignons à ce cas car nous nous appuyons sur des résultats de Troesch qui n'ont été énoncés que sur des corps premiers.

Sur $\mathbb{F}_{2}$, le foncteur $T^{2}$ n'est pas semi-simple, et $\Gamma^{2}$ n'est pas simple (il n'est pas non plus isomorphe à $S^{2}$ ) : on a des suites exactes courtes non scindées

$$
0 \rightarrow \Lambda^{2} \rightarrow \Gamma^{2} \rightarrow \mathrm{Id} \rightarrow 0 \quad \text { et } \quad 0 \rightarrow \Gamma^{2} \rightarrow T^{2} \rightarrow \Lambda^{2} \rightarrow 0,
$$

où la flèche $\Gamma^{2} \rightarrow$ Id («Verschiebung ») est duale du morphisme de Frobenius Id $\rightarrow S^{2}$.

Nous calculerons seulement les groupes $\operatorname{Ext}_{\mathscr{F}}^{*}\left(\mathrm{Id}, I \circ \Gamma^{2}\right)$ et $\operatorname{Ext}_{\mathscr{F}}^{*}\left(\mathrm{Id}, I \circ \Lambda^{2}\right)$, la détermination complète de $\operatorname{Ext}_{\mathscr{G}}^{*}\left(F, I \circ \Gamma^{2}\right)$ ou $\operatorname{Ext}_{\mathscr{F}}^{*}\left(F, I \circ \Lambda^{2}\right)$ semblant hors de portée lorsque $F$ est un foncteur polynomial de degré strictement supérieur à 1.

De fait, les foncteurs $\operatorname{Ext}_{\mathscr{G}}^{*}(\mathrm{Id},-)$ jouissent de la remarquable propriété suivante (qui est vraie sur tout corps fini premier), établie dans [32] (théorème 3.1) :

ThÉORÈme 4.22 (Troesch). - Soient $C^{\bullet}$ un complexe exact d'objets de $\mathcal{F}$ sans terme constant et $F$ un foncteur analytique de $\mathcal{F}$. On a

$$
\operatorname{Ext}_{\mathscr{F}}^{*}\left(\operatorname{Id}, H^{*}\left(F \circ C^{\bullet}\right)\right)=0 .
$$

(Troesch énonce ce résultat seulement pour $F$ polynomial, mais le résultat général s'en déduit aussitôt par passage à la colimite filtrante, puisque Id possède une résolution projective de type fini, d'après un résultat classique de Schwartz, établi par exemple dans [15], proposition 10.1.)

Par conséquent, les deux suites exactes précédentes induisent après postcomposition par $I$ (le caractère analytique de ce foncteur est un fait classique — cf. par exemple [13]) des suites exactes longues :

$$
\cdots \rightarrow \operatorname{Ext}^{i-1}(\operatorname{Id}, I) \rightarrow \operatorname{Ext}^{i}\left(\operatorname{Id}, I \circ \Lambda^{2}\right) \rightarrow \operatorname{Ext}^{i}\left(\operatorname{Id}, I \circ \Gamma^{2}\right) \rightarrow \operatorname{Ext}^{i}(\operatorname{Id}, I) \rightarrow \cdots
$$

et

$$
\cdots \rightarrow \operatorname{Ext}^{i}\left(\mathrm{Id}, I \circ T^{2}\right) \rightarrow \operatorname{Ext}^{i}\left(\mathrm{Id}, I \circ \Lambda^{2}\right) \rightarrow \operatorname{Ext}^{i+1}\left(\mathrm{Id}, I \circ \Gamma^{2}\right) \rightarrow \operatorname{Ext}^{i+1}\left(\mathrm{Id}, I \circ T^{2}\right) \rightarrow \cdots
$$


Mais $\operatorname{Ext}^{i}(\operatorname{Id}, I)$ est nul pour $i \neq 0$ et isomorphe à $\mathbb{F}_{2}$ pour $i=0$, tandis que $\operatorname{Ext}^{i}\left(\operatorname{Id}, I \circ T^{2}\right)=0$ pour tout $i$ d'après la proposition 4.9. On en déduit aussitôt :

THÉORÈME 4.23. - Le $\mathbb{F}_{2}$-espace vectoriel $\operatorname{Ext}_{\mathscr{F}}^{i}\left(\mathrm{Id}, I \circ \Gamma^{2}\right)$ est de dimension 1 pour $i \geq 2$ et nul sinon.

Le $\mathbb{F}_{2}$-espace vectoriel $\operatorname{Ext}_{\mathscr{G}}^{i}\left(\mathrm{Id}, I \circ \Lambda^{2}\right)$ est de dimension 1 pour $i \geq 1$ et nul pour $i=0$.

Remarque 4.24. - De façon similaire, on obtient que $\operatorname{Ext}^{i}\left(\operatorname{Id}, I \circ S^{2}\right)$ est de dimension 1 pour tout $i \in \mathbb{N}$.

On en déduit, par le corollaire 3.32, le résultat suivant.

Corollaire 4.25. - Soient $n$ et $i$ des entiers naturels tels que $n \geq 2 i+7$.

1. L'espace vectoriel $H_{i}\left(O_{n, n}\left(\mathbb{F}_{2}\right), \mathbb{F}_{2}{ }^{2 n}\right)$ est de dimension $i-1$ pour $i \geq 2$ et nul sinon.

2. L'espace vectoriel $H_{i}\left(\operatorname{Sp}_{2 n}\left(\mathbb{F}_{2}\right), \mathbb{F}_{2}{ }^{2 n}\right)$ est de dimension 1 pour $i \geq 1$ et nul sinon.

\section{Appendice A}

\section{Algèbre homologique dans les catégories de foncteurs}

Soit $\mathscr{C}$ une petite catégorie; on rappelle que l'on note $\mathscr{C}$-Mod la catégorie des foncteurs de source $\mathscr{C}$ et de but la catégorie $\mathbf{M o d}_{\mathbb{k}}$ et $\mathbf{M o d}-\mathscr{C}$ pour $\mathscr{C}^{\text {op }}$-Mod. Nous rappelons dans cet appendice quelques résultats homologiques élémentaires et bien connus de cette catégorie, qui se comporte à plusieurs égards comme une catégorie de modules.

Cette catégorie est abélienne, l'exactitude se testant au but. Elle possède toutes limites et colimites, qui se calculent au but. (On renvoie, par exemple, à [17] pour les généralités sur les catégories abéliennes.) C'est même une catégorie de Grothendieck : elle possède un générateur et les colimites filtrantes sont exactes. On peut préciser le premier point de la façon suivante : pour tout objet $i$ de $\mathscr{C}$, on note $P_{i}^{\mathscr{C}}$ le foncteur $\mathbb{k}\left[\operatorname{Hom}_{\mathscr{C}}(i,-)\right]$ (où $\mathbb{k}[E]$ désigne le $\mathbb{k}$-module libre de base $E$ ). Le lemme de Yoneda procure un isomorphisme canonique $\operatorname{Hom}_{\mathscr{C}}\left(P_{i}^{\mathscr{C}}, F\right) \simeq F(i)$ (on note ici $\operatorname{Hom}_{\mathscr{C}}$ pour $\operatorname{Hom}_{\mathscr{C} \text {-Mod }}$; des conventions analogues pour les groupes d'extensions seront utilisées), d'où l'on déduit que les $P_{i}^{\mathscr{C}}$ sont projectifs et engendrent $\mathscr{C}$-Mod. On les appelle parfois générateurs projectifs standard de $\mathscr{C}$-Mod.

Le produit tensoriel au-dessus de $\mathscr{C}$ (cf. par exemple l'appendice $\mathrm{C}$ de [21]) est le foncteur $\underset{\mathscr{C}}{\otimes}:$ Mod- $\mathscr{C} \times \mathscr{C}$-Mod $\rightarrow \operatorname{Mod}_{\mathrm{k}}$ donné ainsi : pour $F \in$ ObMod- $\mathscr{C}$ et $G \in \mathrm{Ob} \mathscr{C}$-Mod, $F \underset{\mathscr{C}}{\otimes} G$ est le quotient de $\underset{i \in \mathrm{Ob}_{\mathscr{C}}}{\bigoplus_{\mathscr{C}}} F(i) \otimes G(i)$ (on rappelle que les produits tensoriels non spécifiés sont pris sur $\mathbb{k}$ ) par le sous-module engendré par les éléments du type $F(f)\left(x^{\prime}\right) \otimes y-x^{\prime} \otimes G(f)(y)$ pour $i \stackrel{f}{\rightarrow} i^{\prime}$ flèche de $\mathscr{C}, y \in G(i)$ et $x^{\prime} \in F\left(i^{\prime}\right)$.

Le bifoncteur $\underset{\mathscr{C}}{\otimes}$ commute aux colimites en chaque variable. Il possède des propriétés de commutativité et d'associativité évidentes. De plus, on a des isomorphismes naturels

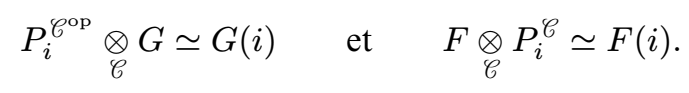

Une autre manière de caractériser le produit tensoriel est l'adjonction suivante :

$$
\operatorname{Hom}_{\mathbb{k}}(F \underset{\mathscr{C}}{\otimes} G, V) \simeq \operatorname{Hom}_{\mathscr{C}}\left(G, \mathcal{H}_{o m_{\mathbb{k}}}(F, V)\right),
$$

$4{ }^{\mathrm{e}}$ SÉRIE - TOME $43-2010-\mathrm{N}^{\mathrm{o}} 3$ 
où, pour $V \in \mathrm{Ob} \operatorname{Mod}_{\mathrm{k}}$ et $F \in \mathrm{Ob} \operatorname{Mod}-\mathscr{C}, \mathscr{H}_{o} m_{\mathrm{k}}(F, V)$ désigne l'objet de $\mathscr{C}$-Mod donné par $C \mapsto \operatorname{Hom}_{\mathbb{k}}(F(C), V)$.

Pour des raisons standard d'algèbre homologique, dériver à gauche le bifoncteur $\underset{\mathscr{C}}{\otimes}$ relativement à l'une ou l'autre des variables donne des résultats canoniquement isomorphes, notés $\operatorname{Tor}_{*}^{\mathscr{C}}$. (On peut aussi définir ces groupes comme l'homologie d'un complexe simplicial explicite - cf. [21], appendice C, par exemple.)

Un foncteur $G \in \mathrm{Ob} \mathscr{C}$-Mod est dit plat $\mathrm{si}-\underset{\mathscr{C}}{\otimes_{C}} G$ est exact. Tout foncteur projectif est plat, et les foncteurs plats sont stables par colimite filtrante. Comme dans le cas des modules, on peut remplacer, pour calculer les groupes de torsion sur $\mathscr{C}$, les résolutions projectives par des résolutions plates.

L'isomorphisme (6) montre que le produit tensoriel au-dessus de $\mathscr{C}$ est en quelque sorte dual du foncteur Hom sur $\mathscr{C}$-Mod. Cela fonctionne particulièrement bien lorsque $\mathbb{k}$ est un corps; on note alors $F^{*}$ pour $\mathcal{H}_{\mathrm{om}_{\mathbb{k}}}(F, \mathbb{k})$, la post-composition de $F$ par le foncteur de dualité des espaces vectoriels, noté $V \mapsto V^{*}$. L'exactitude de cette dualité donne alors un isomorphisme naturel gradué

$$
\operatorname{Tor}^{\mathscr{C}}(F, G)^{*} \simeq \operatorname{Ext}_{\mathscr{C}}\left(G, F^{*}\right)
$$

L'homologie de la catégorie $\mathscr{C}$ à coefficients dans un foncteur $F \in \mathrm{Ob} \mathscr{C}$-Mod est par définition le $\mathbb{k}$-module gradué $\operatorname{Tor}_{*}^{\mathscr{C}}(\mathbb{k}, F)$ (où $\mathbb{k}$ désigne le foncteur de Mod- $\mathscr{C}$ constant en $\mathbb{k})$, qui est noté $H_{*}(\mathscr{C} ; F)$. On remarque que $H_{0}(\mathscr{C} ; F)$ s'identifie canoniquement à la colimite du foncteur $F$. Lorsque $\mathscr{C}$ est la catégorie à un objet associée à un groupe (ou plus généralement un monoïde) $G, F$ se réduit à un $\mathbb{k}[G]$-module et l'on retrouve la notion habituelle d'homologie du groupe $G$ (ou plus généralement de groupes de torsion sur $G$ ). Par ailleurs, le module gradué $H_{*}(\mathscr{C} ; \mathbb{k})$ à coefficients dans le foncteur constant s'identifie à l'homologie singulière du nerf de $\mathscr{C}$.

Nous aurons besoin du résultat suivant déduit de la théorie de l'obstruction des complexes de chaînes exposée par Dold dans [11]; ce n'est qu'une transposition au cas des catégories de foncteurs de type $\mathscr{C}$-Mod de la proposition 1.6 de l'article [24] de Pirashvili sur les $\Gamma$-modules.

Proposition A.1 (Pirashvili). - Soient $F \in \mathrm{Ob}$ C-Mod et $C \bullet$ un complexe de chaines $\mathbb{N}$-gradué d'objets projectifs de Mod- $\mathscr{C}$.

1. Il existe une suite spectrale du premier quadrant

$$
E_{p, q}^{2}=\operatorname{Tor}_{p}^{\mathscr{C}}\left(H_{q}\left(C_{\bullet}\right), F\right) \Rightarrow H_{p+q}\left(C_{\bullet} \underset{\mathscr{C}}{\otimes} F\right)
$$

2. Supposons que

$$
\operatorname{Ext}_{\mathscr{C}^{\circ \mathrm{p}}}^{m-n+1}\left(H_{n}\left(C_{\bullet}\right), H_{m}\left(C_{\bullet}\right)\right)=0 \quad \text { pour } \quad n<m .
$$

Alors la suite spectrale s'effondre au terme $E^{2}$; de plus, il existe une décomposition :

$$
H_{n}\left(C_{\bullet} \otimes_{\mathscr{C}}^{\otimes} F\right) \simeq \bigoplus_{p+q=n} \operatorname{Tor}_{p}^{\mathscr{C}}\left(H_{q}\left(C_{\bullet}\right), F\right)
$$

naturelle en $F$. 
(La suite spectrale en question s'obtient en considérant le complexe double produit tensoriel au-dessus de $\mathscr{C}$ de $C$. et d'une résolution projective de $F$.)

Une généralisation de l'homologie et des groupes de torsion (modulo une hypothèse de projectivité sur les coefficients) sur la catégorie $\mathscr{C}$ est celle d'homologie des bifoncteurs, i.e. des objets de $\mathscr{C}^{\mathrm{op}} \times \mathscr{C}$-Mod. Comme cette notion correspond, dans le cas d'une catégorie à un objet, à celle d'homologie de Hochschild de l'algèbre du monoïde associé, nous noterons $H H_{*}$ cette théorie homologique. Si $B$ est un tel bifoncteur, on définit $H H_{0}(\mathscr{C} ; B)$ comme le quotient de $\bigoplus_{i \in \mathrm{Ob} \mathscr{C}} B(i, i)$ par le sous-module engendré par les éléments du type $B\left(f, \operatorname{Id}_{i}\right)(x)-$ $B\left(\operatorname{Id}_{j}, f\right)(x)$ pour $i, j$ objets de $\mathscr{C}, x \in B(j, i)$ et $f \in \operatorname{Hom}_{\mathscr{C}}(i, j)$. Le foncteur $H H_{0}(\mathscr{C},-)$ est exact à droite; les $\mathbb{k}$-modules $H H_{i}(\mathscr{C} ; B)$ sont par définition l'évaluation en $B$ de ses foncteurs dérivés. Si $B$ est un produit tensoriel extérieur $F \otimes G$ avec $F \in$ Ob Mod- $\mathscr{C}$ et $G \in$ Ob $\mathscr{C}$-Mod, i.e. $B(i, j)=F(i) \otimes G(j)$, et que l'un des foncteurs $F$ et $G$ a pour valeurs des $\mathbb{k}$-modules projectifs, on a $H H_{*}(\mathscr{C} ; B)=\operatorname{Tor}_{*}^{\mathscr{C}}(F, G)$ (c'est vrai sans hypothèse en degré 0 ).

Une autre description de $H H_{*}$ est donnée par l'isomorphisme gradué naturel suivant :

$$
H H_{*}(\mathscr{C} ; B) \simeq \operatorname{Tor}_{*}^{\mathscr{C} \mathrm{op} \times \mathscr{C}}\left(\mathbb{k}\left[\operatorname{Hom}_{\mathscr{C}} \text { op }(-,-)\right], B\right)
$$

(cf. [21], appendice C, par exemple).

L'homologie de $\mathscr{C}$ à coefficients dans un bifoncteur (et de même les groupes de torsion $\operatorname{sur} \mathscr{C}$ ) possède une fonctorialité en $\mathscr{C}:$ si $Q: \mathscr{D} \rightarrow \mathscr{C}$ est un foncteur entre petites catégories et $B$ un objet de $\mathrm{Ob} \mathscr{C}^{\mathrm{op}} \times \mathscr{C}$-Mod, on dispose d'un morphisme canonique $H H_{*}\left(\mathscr{D} ; Q^{*}(B)\right) \rightarrow H H_{*}(\mathscr{C} ; B)$, où l'étoile indique la précomposition et où l'on note par abus encore $Q$ pour $Q^{\mathrm{op}} \times Q: \mathscr{D}^{\mathrm{op}} \times \mathscr{D} \rightarrow \mathscr{C}^{\mathrm{op}} \times \mathscr{C}$. En degré 0 , il se définit en constatant que le morphisme évident

$$
\bigoplus_{j \in \mathrm{Ob} \mathscr{D}} B(Q(j), Q(j)) \rightarrow \bigoplus_{i \in \mathrm{Ob} \mathscr{C}} B(i, i)
$$

induit un morphisme $H H_{0}\left(\mathscr{D} ; Q^{*}(B)\right) \rightarrow H H_{0}(\mathscr{C} ; B)$, qu'on prolonge ensuite en un morphisme de foncteurs homologiques (c'est possible par exactitude de $Q^{*}$ ). C'est un isomorphisme si $Q$ est une équivalence de catégories.

Il existe un analogue de la notion de foncteurs adjoints pour le produit tensoriel au-dessus d'une petite catégorie. Le cas qui nous intéresse est celui correspondant aux extensions de Kan, qu'on peut traiter de façon analogue :

Proposition A.2. - Soient $\mathscr{C}$ et $\mathscr{D}$ des petites catégories et $Q: \mathscr{D} \rightarrow \mathscr{C}$ un foncteur. Il existe un foncteur $Q_{!}:$Mod-D $\rightarrow$ Mod- $\mathscr{C}$, unique à isomorphisme canonique près, tel qu'existe un isomorphisme naturel

$$
F \underset{D}{\otimes} Q^{*}(G) \simeq Q_{!}(F) \underset{\mathscr{C}}{\otimes} G
$$

pour $F \in \mathrm{Ob}$ Mod-D et $G \in \mathrm{Ob} \mathscr{C}$-Mod (où $Q^{*}$ désigne la précomposition par $Q$ ). On peut définir $Q$ ! par

$$
Q_{!}(F)(a)=F \underset{D}{\otimes} Q^{*}\left(P_{a}^{\mathscr{C}}\right)
$$

$4^{\mathrm{e}}$ SÉRIE - TOME $43-2010-\mathrm{N}^{\mathrm{o}} 3$ 
Le foncteur $Q_{!}$est exact à droite; il commute même à toutes les colimites. Ses foncteurs dérivés à gauche sont donnés par

$$
\mathbb{L}_{i} Q_{!}(F)(a)=\operatorname{Tor}_{i}^{\mathscr{D}}\left(F, Q^{*}\left(P_{a}^{\mathscr{C}}\right)\right) .
$$

De plus, le foncteur $Q$ ! est adjoint à gauche au foncteur $\left(Q^{\mathrm{op}}\right)^{*}: \operatorname{Mod}-\mathscr{C} \rightarrow \operatorname{Mod}-\mathscr{D}$; son effet sur les projectifs standard est donné par un isomorphisme naturel

$$
Q_{!}\left(P_{b}^{\mathscr{D}^{\mathrm{op}}}\right) \simeq P_{Q(b)}^{\mathcal{C}^{\mathrm{op}}}
$$

La démonstration, qui consiste en une variation sur le lemme de Yoneda, est laissée au lecteur.

Nous terminons ces rappels par un résultat plus particulier qui intervient dans la section 3.

Proposition A.3. - Soient $X$ un foncteur de $\mathscr{C}^{\text {op }}$ vers la catégorie des ensembles et $\mathscr{C}_{X}$ la catégorie dont les objets sont les couples $(i, x)$ formés d'un objet $i$ de $\mathscr{C}$ et d'un élément $x$ de $X(i)$, les morphismes $(i, x) \rightarrow(j, y)$ étant les morphismes $f: i \rightarrow j$ de $\mathscr{C}$ tels que $X(f)(y)=x$. On note $U: \mathscr{C}_{X} \rightarrow \mathscr{C}$ le foncteur d'oubli (donné sur les objets par $(i, x) \mapsto i$ ), et $\Omega_{X}:$ Mod- $\mathscr{C}_{X} \rightarrow$ Mod-C le foncteur donné par

$$
\Omega_{X}(F)(i)=\bigoplus_{x \in X(i)} F(i, x)
$$

le morphisme $\Omega_{X}(F)(f): \Omega_{X}(F)(i) \rightarrow \Omega_{X}(F)(j)$ induit par un morphisme $f: i \rightarrow j$ de $\mathscr{C}^{\text {op }}$ ayant pour composante $F(i, x) \rightarrow F(j, y)$ l'application induite par $F$ si $X(f)(i)=j$ et 0 sinon.

Il existe un isomorphisme gradué

$$
\operatorname{Tor}^{\mathscr{C} X}\left(F, U^{*}(G)\right) \simeq \operatorname{Tor}^{\mathscr{C}}\left(\Omega_{X}(F), G\right)
$$

naturel en les objets $F$ de Mod- $\mathscr{C}_{X}$ et $G$ de $\mathscr{C}$-Mod.

Ce résultat élémentaire est la traduction en terme de foncteur Tor des adjonctions étudiées dans le $\S 3.1$ de [9]. Le cas du degré 0 se déduit de la formule (11); l'exactitude du foncteur $\Omega_{X}$ permet de propager l'isomorphisme en tout degré homologique.

Le cas des coefficients constants est ce que Loday nomme lemme de Shapiro pour l'homologie des petites catégories ([21], appendice C.12).

\section{Appendice B}

\section{Foncteurs exponentiels}

Dans cet appendice, on s'intéresse uniquement à la catégorie $\mathcal{F}(k)=\mathcal{E}^{f}(k)$-Mod des foncteurs de source $\mathcal{E}^{f}(k)$ et de but $\mathcal{E}(k)$. On notera également $\mathcal{F}_{2}(k)=\mathcal{E}^{f}(k) \times \mathcal{E}^{f}(k)$-Mod.

Les notions que nous rappelons sont classiques : on pourra se référer à [14].

Notons $\pi: \mathscr{E}^{f}(k) \times \mathcal{E}^{f}(k) \rightarrow \mathcal{E}^{f}(k)$ le foncteur de somme directe et $\delta: \mathscr{E}^{f}(k) \rightarrow \mathcal{E}^{f}(k) \times \mathcal{E}^{f}(k)$ le foncteur d'inclusion diagonale. Chacun d'entre eux est adjoint à droite et à gauche à l'autre. Il en résulte que la même propriété vaut pour les foncteurs de précomposition $\pi^{*}: \mathcal{F}(k) \rightarrow \mathcal{F}_{2}(k)$ et $\delta^{*}: \mathcal{F}_{2}(k) \rightarrow \mathcal{F}(k)$. Cette observation simple s'avère tout à fait efficace pour traiter des foncteurs possédant la propriété suivante. 
DÉfinition B.1. - $\quad$ 1. Un foncteur exponentiel de $\mathcal{F}(k)$ est un objet $E$ de $\mathcal{F}(k)$ muni d'un isomorphisme $E(0) \simeq k$ et d'un isomorphisme entre les objets $\pi^{*}(E)$ et $E \otimes E$ de $\mathcal{F}_{2}(k)$ (i.e. d'un isomorphisme $E(U \oplus V) \simeq E(U) \otimes E(V)$ naturel en les objets $U$ et $V$ de $\mathcal{E}_{k}^{f}$ ).

2. Un foncteur exponentiel gradué de $\mathcal{F}(k)$ est une suite $E^{\bullet}=\left(E^{n}\right)_{n \in \mathbb{N}}$ d'objets de $\mathcal{F}(k)$ prenant des valeurs de dimension finie munie d'un isomorphisme $E^{0} \simeq k$ (foncteur constant) et d'un isomorphisme gradué $\pi^{*}\left(E^{\bullet}\right) \simeq E^{\bullet} \otimes E^{\bullet}$ (i.e. pour tout $n \in \mathbb{N}$, d'un isomorphisme $\left.\pi^{*}\left(E^{n}\right) \simeq \bigoplus_{i+j=n} E^{i} \otimes E^{j}\right)$.

3. Soit $E$ un foncteur exponentiel, éventuellement gradué, de $\mathcal{F}(k)$. Si la structure exponentielle de $E$ est compatible aux isomorphismes d'associativité de la somme directe et du produit tensoriel ${ }^{(2)}$, on dit que $E$ est un foncteur exponentiel de Hopf.

Par exemple, les foncteurs projectifs standard de $\mathcal{F}(k)$ ou le foncteur injectif $I$ (cf. notation 3.30) sont des foncteurs exponentiels de Hopf. Les foncteurs puissance symétrique $S^{*}$, puissance divisée $\Gamma^{*}$ (dual gradué du précédent) et puissance extérieure $\Lambda^{*}$ sont exponentiels gradués de Hopf. (Cela provient directement des propriétés universelles qui les caractérisent.) De plus, $S^{*}$ et $\Gamma^{*}$ sont commutatifs et $\Lambda^{*}$ est anticommutatif (au sens gradué) en un sens évident, qui est précisé dans [14], page 675 .

Noter que les composantes d'un foncteur exponentiel gradué sont des foncteurs polynomiaux.

Remarque B.2. - Le foncteur gradué puissance tensorielle $T^{*}$ n'est pas exponentiel, mais il possède une propriété analogue dont on peut se servir dans les calculs d'une façon similaire à la propriété exponentielle : la formule du binôme se traduit par un isomorphisme

$$
\pi^{*}\left(T^{n}\right) \simeq \bigoplus_{i+j=n}\left(T^{i} \otimes T^{j}\right) \underset{\mathfrak{S}_{i} \times \mathfrak{S}_{j}}{\otimes} k\left[\mathfrak{S}_{n}\right]
$$

où le groupe symétrique $\mathfrak{S}_{n}$ agit par permutation des facteurs du produit tensoriel et le groupe $\mathfrak{S}_{i} \times \mathfrak{S}_{j}$ est plongé de manière usuelle dans $\mathfrak{S}_{i+j}$.

La proposition suivante est une partie du théorème 1.7 de [14]; sa démonstration repose essentiellement sur l'adjonction entre les foncteurs $\pi^{*}$ et $\delta^{*}$ évoquée plus haut et son prolongement aux groupes d'extensions (qui découle de leur exactitude).

(2) Cela signifie que le diagramme évident d'isomorphismes

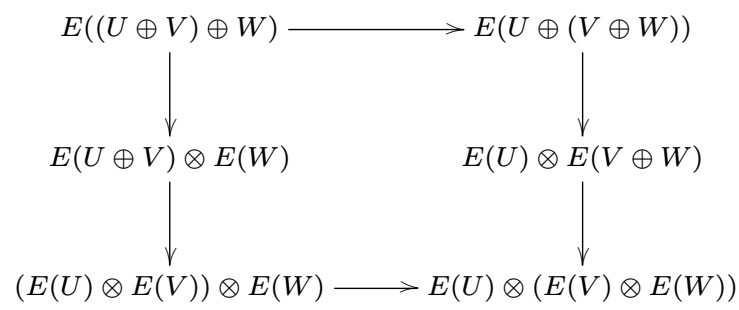

commute pour tous $U, V, W \in \mathrm{Ob} \mathcal{E}^{f}(k)$.

$4^{\text {e }}$ SÉRIE - TOME $43-2010-\mathrm{N}^{\mathrm{o}} 3$ 
Proposition B.3. - Soient $E^{*}$ un foncteur exponentiel gradué et $F, G$ deux foncteurs de $\mathcal{F}(k)$. Il existe, pour tout $n \in \mathbb{N}$, un isomorphisme gradué

$$
\operatorname{Ext}_{\mathscr{F}(k)}^{*}\left(E^{n}, F \otimes G\right) \simeq \bigoplus_{i+j=n} \operatorname{Ext}_{\mathscr{F}(k)}^{*}\left(E^{i}, F\right) \otimes \operatorname{Ext}_{\mathscr{F}(k)}^{*}\left(E^{j}, G\right)
$$

naturel en $F$ et $G$.

(Autrement dit, on dispose d'un isomorphisme bigradué $\operatorname{Ext}_{\mathscr{G}(k)}^{*}\left(E^{\bullet}, F \otimes G\right) \simeq$ $\operatorname{Ext}_{\mathscr{F}(k)}^{*}\left(E^{\bullet}, F\right) \otimes \operatorname{Ext}_{\mathscr{F}(k)}^{*}\left(E^{\bullet}, G\right)$.)

Si $E$ est un foncteur exponentiel, éventuellement gradué, on dispose de morphismes $k \simeq E(0) \rightarrow E$, appelé unité, $E \rightarrow E(0) \simeq k$, appelé co-unité, $E \otimes E=\delta^{*}(E \otimes E) \simeq$ $\delta^{*} \pi^{*}(E) \rightarrow E$ (induit par la co-unité de l'adjonction), appelé produit, $E \rightarrow E \otimes E=$ $\delta^{*}(E \otimes E) \simeq \delta^{*} \pi^{*}(E)$, appelé coproduit, et $E(-\mathrm{Id}): E \rightarrow E$, appelé antipode. On vérifie facilement (cf. [14]) que ces applications font de $E$ une algèbre de Hopf (graduée connexe si $E$ est gradué) dans la catégorie monoïdale symétrique $(\mathcal{F}(k), \otimes, k)$ lorsque $E$ est un foncteur exponentiel de Hopf (c'est en fait une caractérisation des foncteurs exponentiels de Hopf).

Ce type de structure est utile pour définir des produits de convolution. Supposons en effet que $E^{\bullet}$ est un foncteur exponentiel de Hopf et $F$ un foncteur de $\mathcal{F}(k)$ muni d'une structure d'algèbre. Alors $\operatorname{Ext}_{\mathscr{F}(k)}^{*}\left(E^{\bullet}, F\right)$ est une algèbre bigraduée pour le produit, dit de convolution,

$$
\text { * }: \operatorname{Ext}_{\mathscr{G}(k)}^{*}\left(E^{\bullet}, F\right) \otimes \operatorname{Ext}_{\mathscr{F}(k)}^{*}\left(E^{\bullet}, F\right) \simeq \operatorname{Ext}_{\mathscr{F}(k)}^{*}\left(E^{\bullet}, F \otimes F\right) \rightarrow \operatorname{Ext}_{\mathscr{F}(k)}^{*}\left(E^{\bullet}, F\right),
$$

où l'isomorphisme est donné par la proposition B.3 et la seconde flèche est induite par le produit de $F$; l'unité de ce produit est donnée par l'élément de $\operatorname{Hom}_{\mathcal{G}(k)}\left(E^{0}, F\right) \simeq F(0)$ unité de l'algèbre $F$.

Si $F$ est un foncteur muni d'une structure de cogèbre, on définit de même un coproduit sur $\operatorname{Ext}_{\mathscr{F}(k)}^{*}\left(E^{\bullet}, F\right)$ comme le morphisme

$$
\operatorname{Ext}_{\mathscr{F}(k)}^{*}\left(E^{\bullet}, F\right) \rightarrow \operatorname{Ext}_{\mathscr{T}(k)}^{*}\left(E^{\bullet}, F \otimes F\right) \simeq \operatorname{Ext}_{\mathscr{F}(k)}^{*}\left(E^{\bullet}, F\right) \otimes \operatorname{Ext}_{\mathscr{G}(k)}^{*}\left(E^{\bullet}, F\right)
$$

où la première flèche provient du coproduit de $F$. Lorsque $F$ est muni d'une structure d'algèbre de Hopf dans $\mathcal{F}(k)$, on obtient sur $\operatorname{Ext}_{\mathscr{G}(k)}^{*}\left(E^{\bullet}, F\right)$ une structure de $k$-algèbre de Hopf bigraduée (l'antipode étant induite par celle de $F$ ), connexe si $F(0)=k$.

On utilise, au paragraphe 4.2, les deux propositions suivantes où intervient la convolution.

Proposition B.4. - Soit $E^{\bullet}$ un foncteur exponentiel de Hopf gradué, commutatif ou anticommutatif.

1. Pour tout objet $V$ de $\mathcal{E}^{f}(k), E^{\bullet}(V)$ est naturellement une $k$-algèbre de Hopf graduée.

2. Si $f: V \rightarrow W$ est une flèche de $\mathcal{E}^{f}(k)$, alors $E^{\bullet}(f): E^{\bullet}(V) \rightarrow E^{\bullet}(W)$ est un morphisme de k-algèbres de Hopf graduées.

Si $g: V \rightarrow W$ est une autre flèche de $\mathcal{E}^{f}(k)$, on a $E^{\bullet}(f+g)=E^{\bullet}(f) * E^{\bullet}(g)$.

Cette conséquence facile des définitions est laissée au lecteur. 
Remarque B.5. - La nécessité d'une hypothèse d'(anti)commutativité est omise dans l'article [14]; elle a été remarquée par Touzé dans [30] (cf. sa remarque 5.6). On renvoie d'une manière générale le lecteur à la section 5 de [30] où toutes les questions de compatibilité (et parfois de regraduation) nécessaires dans les énoncés relatifs aux structures exponentielles sont traitées avec soin.

Proposition B.6. - Soient $E^{\bullet}$ un foncteur exponentiel gradué de Hopf commutatif ou anticommutatif de $\mathcal{F}(k)$ et $F$ un foncteur muni d'une structure d'algèbre (resp. d'algèbre de Hopf) dans la catégorie monoïdale symétrique $(\mathcal{F}(k), \otimes, k)$.

1. Si A est un foncteur sans terme constant (i.e. $A(0)=0$ ) de $\mathcal{F}(k)$, la structure d'algèbre (resp. d'algèbre de Hopf) de F induit une structure d'algèbre (resp. d'algèbre de Hopf) sur $F \circ A$.

2. Si $u: A \rightarrow B$ est un morphisme entre foncteurs sans terme constant de $\mathcal{F}(k)$, $\operatorname{Ext}^{*}\left(E^{\bullet}, F(u)\right): \operatorname{Ext}^{*}\left(E^{\bullet}, F \circ A\right) \rightarrow \operatorname{Ext}^{*}\left(E^{\bullet}, F \circ B\right)$ est un morphisme de $k$-algèbres (resp. de k-algèbres de Hopf) bigraduées. Ce morphisme sera noté $h(u)$.

3. Si $v: A \rightarrow B$ est un autre tel morphisme et que $F$ est un foncteur exponentiel de Hopf commutatif ou anticommutatif, on a $h(u+v)=h(u) * h(v)$.

Démonstration. - Les deux premières assertions sont immédiates. Pour la dernière, on écrit $u+v$ comme la composée $A \hookrightarrow A \oplus A \stackrel{u \oplus v}{\longrightarrow} B \oplus B \rightarrow B$, où le premier morphisme est la diagonale et le dernier la somme, puis on considère le diagramme commutatif suivant

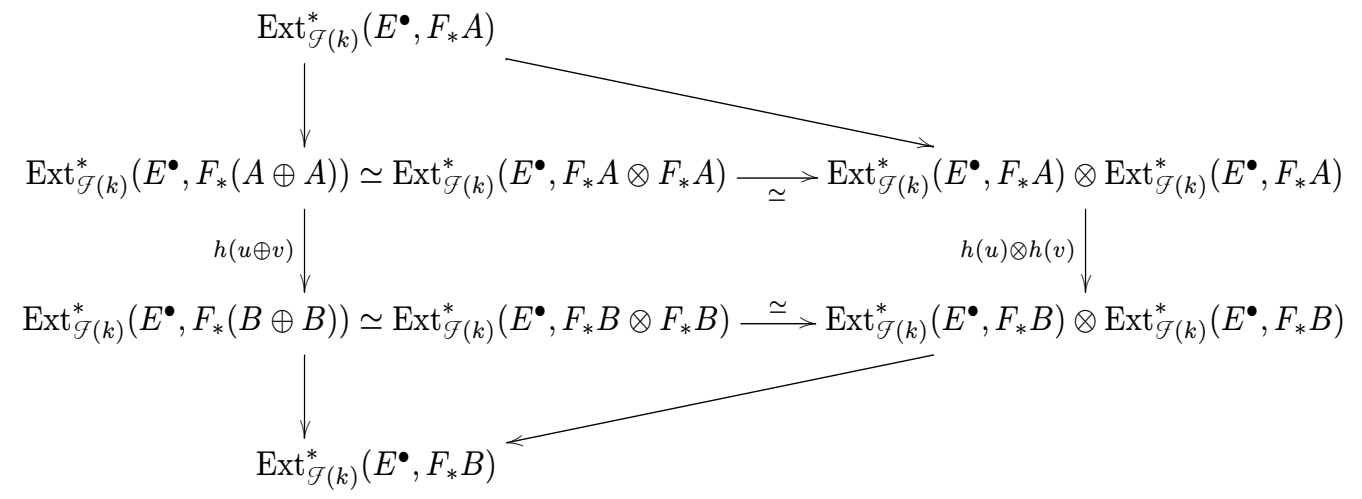

où l'on note pour alléger $F_{*}$ la postcomposition par $F$ et dans lequel les flèches verticales ou obliques non spécifiées sont induites par coproduit ou produit : le chemin vertical gauche fournit $h(u+v)$ et celui de droite $h(u) * h(v)$.

\section{Appendice $\mathbf{C}$}

\section{Inversion de Möbius et équivalences de Morita}

Cet appendice a pour but de montrer que l'argument donné par le second auteur pour obtenir l'équivalence de catégories du théorème 4.2 de [33] :

$$
\operatorname{Sp}\left(\delta_{q}^{\mathrm{deg}}\left(\mathbb{F}_{2}\right)\right)-\mathbf{M o d} \simeq \prod_{V \in \varnothing} \mathbb{F}_{2}[O(V)]-\text { Mod }
$$


où $\operatorname{Sp}\left(\mathcal{E}_{q}^{\mathrm{deg}}\left(\mathbb{F}_{2}\right)\right)$ est la catégorie de Burnside associée à $\mathcal{E}_{q}^{\mathrm{deg}}\left(\mathbb{F}_{2}\right)$ dont on rappelle la définition en C.4 et $\&$ est un ensemble de représentants des classes d'isométrie des objets de $\mathcal{E}_{q}^{\mathrm{deg}}\left(\mathbb{F}_{2}\right)$, s'adapte facilement à d'autres situations pour donner des équivalences de catégories non triviales. L'une d'entre elles intervient dans la preuve du théorème 3.21.

Cet argument repose sur la combinaison de l'inversion de Möbius et du théorème de Morita-Freyd dont on rappelle ici les énoncés.

La fonction de Möbius $\mu_{X}$ d'un ensemble fini partiellement ordonné $(X, \leq)$ est définie par:

$$
\begin{aligned}
\mu_{X}(x, x) & =1 & & \text { pour tout } x \text { dans } X \\
\sum_{x \leq z \leq y} \mu_{X}(x, z) & =0 & & \text { pour tous } x<y \text { dans } X .
\end{aligned}
$$

ThÉORÈme C.1 (Théorème 3.9.2 de [28]). - Soit $(X, \leq)$ un ensemble fini partiellement ordonné, dans lequel toute paire $\{x, y\}$ a une borne inférieure $x \wedge y$. Supposons que $X$ a un plus grand élément $M$. Soit $R$ un anneau (avec unité $1_{R}$ ). Supposons que $\alpha \mapsto e_{\alpha}$ est une application de $X$ dans $R$ vérifiant les propriétés suivantes : $e_{\alpha} e_{\beta}=e_{\alpha \wedge \beta}$ pour tout $\alpha, \beta \in X$, et $e_{M}=1_{R}$. Pour $\alpha \in X$, on définit :

$$
f_{\alpha}=\sum_{\beta \leq \alpha} \mu_{X}(\beta, \alpha) e_{\beta},
$$

où $\mu_{X}$ est la fonction de Möbius de $X$. Alors les éléments $f_{\alpha}$, pour $\alpha \in X$, sont des idempotents orthogonaux de $R$, dont la somme est égale à $1_{R}$.

Le théorème suivant, dû à Freyd, doit être vu comme une forme générale, «à plusieurs objets », du théorème classique de Morita sur l'équivalence des catégories de modules.

ThÉORÈme C.2 (Morita-Freyd). - Soient G une catégorie de Grothendieck $\mathbb{k}$-linéaire (i.e. enrichie sur les $\mathbb{k}$-modules) et $\mathscr{G}$ une sous-catégorie pleine petite de $\mathscr{C}$ dont les objets sont projectifs de type fini et engendrent $\mathscr{C}$. Alors $\mathscr{C}$ est équivalente à la catégorie des foncteurs $\mathbb{k}$-linéaires de $\mathscr{G}^{\mathrm{op}}$ dans $\mathbf{M o d}_{\mathbb{k}}$.

On commence par appliquer ces outils à la catégorie $\Gamma$ des ensembles finis pointés et on montre que cela permet de retrouver le théorème à la Dold-Kan démontré par Pirashvili dans l'article [23], qu'on utilisera dans l'appendice E. On étudie ensuite le cas, très proche de celui considéré dans [33], de la catégorie de Burnside associée à une «bonne catégorie » et qui fournit une équivalence de catégories qu'on emploie dans le paragraphe 3.2.

On désigne par $\Omega$ la catégorie des ensembles finis avec surjections. On note $(-)_{+}$l'adjoint à gauche au foncteur d'oubli de $\Gamma$ vers la catégorie des ensembles finis : pour $E$ un ensemble fini, $E_{+}$s'obtient en adjoignant un point de base externe à $E$.

ThÉorème C.3 (Pirashvili). - Il existe une équivalence de catégories cr : Mod- $\Gamma \rightarrow$ Mod- $\Omega$ telle que

$$
\operatorname{cr}(F)(E)=\operatorname{Coker}\left(\bigoplus_{e \in E} F\left(E_{e}\right) \rightarrow F\left(E_{+}\right)\right)
$$

morphisme induit par les surjections $E_{+} \rightarrow E_{e}$ égales à l'identité hors du point de base, où $E_{e}$ désigne l'ensemble E pointé par e. 
De plus, le foncteur $i_{!}: \Omega-\mathbf{M o d} \rightarrow \Gamma-\mathbf{M o d}$ tel que

$$
G \underset{\Gamma}{\otimes} i_{!}(F) \simeq \operatorname{cr}(G) \underset{\Omega}{\otimes} F
$$

est donné par

$$
i_{!}(F)(E)=\bigoplus_{E^{\prime} \subset E \backslash\{*\}} F\left(E^{\prime}\right)
$$

Démonstration. - Soit $E$ un objet de $\Gamma$ de point de base $*$. On considère l'ensemble $\mathfrak{p}(E)$ de ses sous-objets (i.e. de ses sous-ensembles contenant le point de base) ordonné par l'inclusion ; $\mathfrak{p}(E)$ admet pour plus grand élément $E$ et toute paire d'éléments de $\mathfrak{p}(E),\{A, B\}$ admet pour plus grand minorant l'intersection $A \cap B$. Soit $R=\mathbb{k}\left[\operatorname{End}_{\Gamma}(E)\right]$ la $\mathbb{k}$-algèbre du

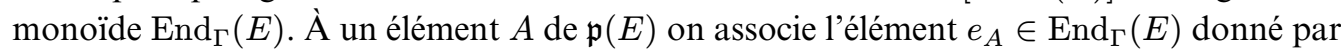
$e_{A}(x)=x$ si $x \in A$ et $e_{A}(x)=*$ sinon. On a $e_{E}=\operatorname{Id}_{E}$ et $e_{A} e_{B}=e_{A \cap B}$. Par conséquent, par le théorème C.1 les éléments $f_{A}$ définis par:

$$
f_{A}=\sum_{B \subset A} \mu_{\mathfrak{p}(E)}(B, A) e_{B}
$$

sont des idempotents orthogonaux de $R$ de somme égale à $\operatorname{Id}_{E}$. On en déduit la décomposition

$$
P_{E}^{\Gamma} \simeq \bigoplus_{A \in \mathfrak{p}(E)} f_{A} P_{E}^{\Gamma}
$$

où les $P_{E}^{\Gamma}$ désignent les projectifs standard de $\Gamma$-Mod. Les foncteurs $P_{E}^{\Gamma}$ sont de type fini et donc leurs facteurs directs $f_{A} P_{E}^{\Gamma}$ forment un ensemble de générateurs projectifs de type fini de $\Gamma$-Mod.

Afin d'appliquer le théorème C.2, nous avons besoin d'identifier les modules suivants

$$
\operatorname{Hom}\left(f_{A} P_{E}^{\Gamma}, f_{B} P_{E}^{\Gamma}\right) \simeq f_{B} \mathbb{k}\left[\operatorname{End}_{\Gamma}(E)\right] f_{A} .
$$

Pour cela, on fait les observations suivantes, pour tous $A \in \mathfrak{p}(E)$ et $t \in \operatorname{End}_{\Gamma}(E)$ :

1. $e_{A} t=t e_{t^{-1}(A)}$

2. $t=t e_{B}$, où $B$ désigne l'élément de $\mathfrak{p}(E)$ réunion du point de base et de l'ensemble complémentaire de $t^{-1}(*)$ dans $E$.

Du premier point on déduit

$$
f_{A} t=t \sum_{B \subset A} \mu_{\mathfrak{p}(E)}(B, A) e_{t^{-1}(B)}
$$

puis, compte tenu de ce que $e_{C} f_{D}=f_{D}$ si $D \subset C, 0$ sinon,

$$
f_{A} t f_{A^{\prime}}=t \sum_{t\left(A^{\prime}\right) \subset B \subset A} \mu_{\mathfrak{p}(E)}(B, A) f_{A^{\prime}}=\left\{\begin{array}{l}
t f_{A^{\prime}} \operatorname{sit} t\left(A^{\prime}\right)=A, \\
0 \text { sinon. }
\end{array}\right.
$$

Du second vient que $t f_{A^{\prime}}$ est nul sauf si $A^{\prime} \subset\left(E \backslash t^{-1}(*)\right) \cup\{*\}$. Cette condition signifie que $A^{\prime}$ induit une fonction de $A^{\prime} \backslash\{*\}$ dans $E \backslash\{*\}$, ou une surjection de $A^{\prime} \backslash\{*\}$ vers $A \backslash\{*\}$ si $t\left(A^{\prime}\right)=A$.

Des deux observations précédentes, et du fait que $t f_{A^{\prime}}$, comme les $t e_{B}$ pour $B \subset A^{\prime}$, ne dépend que de la restriction de $t$ à $A^{\prime}$, on déduit une application linéaire surjective

$$
\mathbb{k}\left[\operatorname{Surj}\left(A^{\prime} \backslash\{*\}, A \backslash\{*\}\right)\right] \rightarrow f_{A} \mathbb{k}\left[\operatorname{End}_{\Gamma}(E)\right] f_{A^{\prime}},
$$

$4^{\mathrm{e}}$ SÉRIE - TOME $43-2010-\mathrm{N}^{\mathrm{o}} 3$ 
où Surj désigne l'ensemble des fonctions surjectives entre deux ensembles.

Un argument de rang montre que cette application est en fait bijective : en effet, la somme directe des $\mathbb{k}$-modules libres $f_{A} \mathbb{k}\left[\operatorname{End}_{\Gamma}(E)\right] f_{A^{\prime}}$ lorsque $A$ et $A^{\prime}$ parcourent $\mathfrak{p}(E)$ est isomorphe à $\mathbb{k}\left[\operatorname{End}_{\Gamma}(E)\right]$. On conclut par la bijection

$$
\operatorname{End}_{\Gamma}(E) \simeq \bigsqcup_{\left(A, A^{\prime}\right) \in \mathfrak{p}(E)^{2}} \operatorname{Surj}\left(A^{\prime} \backslash\{*\}, A \backslash\{*\}\right)
$$

obtenue en associant à un endomorphisme $t$ de $E$ les éléments $A=t(E)$ et $A^{\prime}=\left(E \backslash t^{-1}(*)\right) \cup\{*\}$ de $\mathfrak{p}(E)$ et la surjection $A^{\prime} \backslash\{*\} \rightarrow A \backslash\{*\}$ induite par $t$.

On constate d'autre part que l'isomorphisme (14) est compatible à la composition en un sens évident. Il nous reste à expliciter les équivalences de catégories données par le théorème de Freyd-Morita. La décomposition (14) fournit un isomorphisme

$$
\left(i_{!}(X)\right)(E) \simeq \bigoplus_{A \in \mathfrak{p}(E)} X(A \backslash\{*\})=\bigoplus_{\substack{E^{\prime} \subset E \\ * \notin E^{\prime}}} X\left(E^{\prime}\right)
$$

pour tout objet $X$ de $\Omega$-Mod et tout objet $E$ de $\Gamma$. On vérifie facilement que la fonctorialité s'obtient en écrivant $i_{!}(X)(E)$ comme conoyau de l'injection naturelle

$$
\bigoplus_{A \in \mathfrak{p}(E)} X(A) \hookrightarrow \bigoplus_{A \subset E} X(A)
$$

(Autrement dit, la composante $X\left(E^{\prime}\right) \rightarrow X\left(F^{\prime}\right)$ de $X(f)$, pour $f: E \rightarrow F$ morphisme de $\Gamma$, est induite par $f$ si $f\left(E^{\prime}\right)=F^{\prime}$ et nulle sinon.)

La formule pour cr s'obtient de manière analogue.

Avant d'appliquer cette méthode au cas dont on se sert au paragraphe 3.2, on rappelle la définition de la catégorie de Burnside d'une catégorie admettant des produits fibrés. Cette terminologie est issue de la théorie des représentations; la notation Sp provient du terme anglais span.

DÉfinition C.4 ([1]). - Soit $\mathscr{C}$ une catégorie admettant des produits fibrés; la catégorie de Burnside de $\mathscr{C}$, notée $\operatorname{Sp}(\mathscr{C})$, est définie de la manière suivante :

1. les objets de $\operatorname{Sp}(\mathscr{C})$ sont ceux de $\mathscr{C}$;

2. pour $A$ et $B$ deux objets de $\operatorname{Sp}(\mathscr{C}), \operatorname{Hom}_{\operatorname{Sp}(\mathscr{C})}(A, B)$ est l'ensemble des classes d'équivalence de diagrammes dans $\mathscr{C}$ de la forme $A \stackrel{f}{\leftarrow} D \stackrel{g}{\rightarrow} B$, pour la relation d'équivalence qui identifie les deux diagrammes $A \stackrel{f}{\leftarrow} D \stackrel{g}{\rightarrow} B$ et $A \stackrel{u}{\leftarrow} D^{\prime} \stackrel{v}{\rightarrow} B$ s'il existe un isomorphisme $\alpha: D \rightarrow D^{\prime}$ rendant le diagramme suivant commutatif :

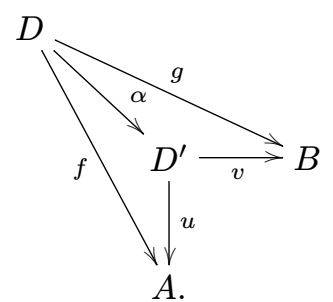


Le morphisme de $\operatorname{Hom}_{\mathrm{Sp}(\mathscr{C})}(A, B)$ représenté par le diagramme $A \stackrel{f}{\leftarrow} D \stackrel{g}{\rightarrow} B$ sera noté $[A \stackrel{f}{\leftarrow} D \stackrel{g}{\rightarrow} B]$.

3. Pour deux morphismes $T_{1}=[A \leftarrow D \rightarrow B]$ et $T_{2}=\left[B \leftarrow D^{\prime} \rightarrow C\right]$ la composition est donnée par :

$$
T_{2} \circ T_{1}=\left[A \leftarrow D \underset{B}{\times} D^{\prime} \rightarrow C\right] .
$$

On appelle foncteur de Mackey non additif depuis $\mathscr{C}$ un foncteur $\operatorname{Sp}(\mathscr{C}) \rightarrow \mathbf{M o d}_{\mathrm{k}}$.

Donnons quelques notations et définitions supplémentaires dans le cas où la catégorie $\mathscr{C}$ est petite et où tous ses morphismes sont des monomorphismes. Un sous-objet d'un objet $i$ de $\mathscr{C}$ est une classe d'équivalence de morphisme de but $i$ pour la relation identifiant $f: j \rightarrow i$ à $f^{\prime}: j^{\prime} \rightarrow i$ lorsqu'il existe un isomorphisme $g: j \stackrel{\simeq}{\longrightarrow} j^{\prime}$ tel que $f=f^{\prime} g$. On notera $\mathfrak{p}(i)$ l'ensemble des sous-objets de $i$; on supposera de plus effectué le choix d'un représentant dans chaque classe (ces représentants seront spécifiés par la notation $\hookrightarrow$ ), auquel on identifiera celle-ci. L'ensemble $\mathfrak{p}(i)$ est muni d'une relation d'ordre notée $\subset$ définie par $k \subset j$ lorsque le morphisme $k \hookrightarrow i$ se factorise (de manière unique puisque les flèches de $\mathscr{C}$ sont des monomorphismes) par $j \hookrightarrow i$; l'existence de produits fibrés dans $\mathscr{C}$ assure que deux éléments $j$ et $k$ de cet ensemble ordonné possèdent une borne inférieure notée $j \cap k$.

L'image d'un morphisme de $\mathscr{C}$ est par définition sa classe dans l'ensemble des sous-objets de son but. Si $t=[i \stackrel{u}{\leftarrow} k \stackrel{v}{\rightarrow} j]$ est un élément de $\operatorname{Hom}_{\mathrm{Sp}(\mathscr{C})}(i, j)$, l'image de $u$ (resp. $v$ ), qui ne dépend pas du choix des représentants, est notée $\operatorname{coim}(t)$ (resp. $i m(t)$ ); $t$ possède une unique écriture $t=[i \hookleftarrow \operatorname{coim}(t) \stackrel{\bar{t}}{\rightarrow} j]$.

Pour $f \in \operatorname{Hom}_{\mathscr{C}}\left(i, i^{\prime}\right), j \in \mathfrak{p}(i)$ et $j^{\prime} \in \mathfrak{p}\left(i^{\prime}\right)$, on note $f(j)=i m\left(j \hookrightarrow i \stackrel{f}{\rightarrow} i^{\prime}\right)$ et $f^{-1}\left(j^{\prime}\right)$ l'élément de $\mathfrak{p}(i)$ donné par le produit fibré de $f$ et de $j^{\prime} \hookrightarrow j$.

ThÉORÈme C.5 (Cf. [33], théorème 4.2). - Soit $\mathscr{C}$ une petite catégorie admettant des produits fibrés, dont tous les morphismes sont des monomorphismes et telle que l'ensemble $\mathfrak{p}(i)$ est fini pour tout $i \in \mathrm{Ob} \mathscr{C}$. Notons $\mathscr{C}_{\text {iso }}$ la sous-catégorie de $\mathscr{C}$ ayant les mêmes objets et pour morphismes les isomorphismes de $\mathscr{C}$. Il existe une équivalence de catégories

$$
\xi: \mathscr{C}_{\text {iso }}-\mathbf{M o d} \stackrel{\simeq}{\longrightarrow} \mathrm{Sp}(\mathscr{C}) \text {-Mod }
$$

telle que $\xi(M)(i)=\bigoplus_{j \in \mathfrak{p}(i)} M(j)$ et qu'un morphisme $t \in \operatorname{Hom}_{\mathrm{Sp}(\mathscr{C})}\left(i, i^{\prime}\right)$ induit l'application $\xi(M)(i) \rightarrow \xi(M)\left(i^{\prime}\right)$ dont la composante $M(j) \rightarrow M\left(j^{\prime}\right)$ (pour $j \in \mathfrak{p}(i)$ et $j^{\prime} \in \mathfrak{p}\left(i^{\prime}\right)$ ) est $M(\tilde{t})$ si $j \subset \operatorname{coim}(t)$ et que $\bar{t}(j)=j^{\prime}$, de sorte que $\bar{t}$ induit un isomorphisme $\tilde{t}: j \stackrel{\simeq}{\longrightarrow} j^{\prime}$, et est nulle sinon.

Démonstration. - Reprenant les arguments de [33], on procède de manière très analogue à la démonstration du théorème C.3.

Soient $c$ un objet de $\mathscr{C}$ et $i$ un élément de $\mathfrak{p}(c)$; on note $e_{i}^{c}$ (ou simplement $e_{i}$ ) l'idempotent $[c \hookleftarrow i \hookrightarrow c]$ de $\operatorname{End}_{\mathrm{Sp}(\mathscr{C})}(c)$. On a $e_{c}^{c}=\operatorname{Id}_{c}$ et $e_{i}^{c} \cdot e_{j}^{c}=e_{i \cap j}^{c}$, ce qui permet d'appliquer le théorème C.1 pour obtenir une famille complète d'idempotents orthogonaux

$$
f_{a}^{c}=\sum_{i \subset a} \mu_{\mathfrak{p}(c)}(i, a) e_{i}^{c}
$$

de l'anneau $\mathbb{k}\left[\operatorname{End}_{\mathrm{Sp}(\mathscr{C})}(c)\right]$.

$4^{\text {e }}$ SÉRIE - TOME $43-2010-\mathrm{N}^{\mathrm{o}} 3$ 
On remarque maintenant que pour tout morphisme $t \in \operatorname{Hom}_{\mathrm{Sp}(\mathscr{C})}(c, d)$, on a :

1. $t e_{i}^{c}=e_{\bar{t}(i)}^{d} t$ pour $i \subset \operatorname{coim}(t)$;

2. $t e_{\text {coim }(t)}^{c}=t$.

Le second point montre que $t f_{a}^{c}=t e_{\operatorname{coim}(t)}^{c} f_{a}^{c}=0$ sauf si $a \subset \operatorname{coim}(t)$.

On déduit par ailleurs du premier point que, sous l'hypothèse $a \subset \operatorname{coim}(t)$ :

$$
f_{b}^{d} t f_{a}^{c}=\sum_{i \subset a} \mu_{\mathfrak{p}(c)}(i, a) f_{b}^{d} e_{\bar{t}(i)}^{d} t=\sum_{\substack{i \subset a \\
b \subset \bar{t}(i)}} \mu_{\mathfrak{p}(c)}(i, a) f_{b}^{d} t=\left\{\begin{array}{l}
f_{b}^{d} t \text { si } \bar{t}(a)=b, \\
0 \text { sinon. }
\end{array}\right.
$$

Par conséquent, $f_{b}^{d} t f_{a}^{c}$ est nul sauf si $a \subset \operatorname{coim}(t)$ et que $\bar{t}$ induit un isomorphisme $\tilde{t}$ de $a$ vers $b$. De surcroît, comme $f_{b}^{d}=f_{b}^{d} e_{b}^{d}$ et $f_{a}^{c}=e_{a}^{c} f_{a}^{c}, f_{b}^{d} t f_{a}^{c}$ ne dépend alors que de $e_{b}^{d} t e_{a}^{c}$, c'est-à-dire de $\tilde{t}$. Il s'ensuit que l'application linéaire

$$
\mathbb{k}\left[\operatorname{Hom}_{\mathscr{C}_{\text {iso }}}(a, b)\right] \rightarrow f_{b}^{d} \mathbb{k}\left[\operatorname{Hom}_{\mathrm{Sp}(\mathscr{C})}(c, d)\right] f_{a}^{c} \quad[u] \mapsto f_{b}^{d}[c \hookleftarrow a \stackrel{u}{\rightarrow} b \hookrightarrow d] f_{a}^{c}
$$

est surjective. On montre que c'est en fait un isomorphisme par un argument de rang analogue à celui utilisé pour le théorème C.3, et l'on termine aussi la démonstration de la même façon.

\section{Appendice D}

\section{Quelques résultats d'annulation en homologie des foncteurs}

Cet appendice a pour objet de rappeler brièvement deux résultats d'annulation homologique utilisés de manière cruciale dans la démonstration du théorème 3.21. On s'y donne un corps fini $k$, qui sera souvent sous-entendu dans les notations.

Définition D.1. - $\quad$ On désigne par $\mathcal{E}_{\text {surj }}^{f}\left(\right.$ resp. $\left.\mathcal{E}_{\text {inj }}^{f}\right)$ la sous-catégorie de la catégorie $\mathcal{E}^{f}$ des $k$-espaces vectoriels de dimension finie ayant les mêmes objets et les surjections (resp. les injections) pour morphismes.

- On désigne par $\mathcal{E}_{\mathscr{g} r}^{f}$ la catégorie des couples $(V, W)$ formés d'un espace vectoriel de dimension finie $V$ et d'un sous-espace vectoriel $W$, avec pour morphismes $(V, W) \rightarrow\left(V^{\prime}, W^{\prime}\right)$ les applications linéaires $f: V \rightarrow V^{\prime}$ telles que $f(W)=W^{\prime}$.

- On note $\iota: \operatorname{Mod}-\mathcal{E}^{f} \rightarrow \operatorname{Mod}-\mathcal{E}_{\mathscr{g} r}^{f}$ le foncteur de précomposition par $\mathcal{E}_{\mathscr{G} r}^{f} \rightarrow \mathcal{E}^{f} \quad(V, W) \mapsto V$.

- On note $\rho: \operatorname{Mod}-\delta_{\text {surj }}^{f} \rightarrow \operatorname{Mod}-\delta_{g_{r}}^{f}$ la précomposition par $\varepsilon_{g_{r}}^{f} \rightarrow \delta_{\text {surj }}^{f}(V, W) \mapsto W$.

- On note $\kappa: \operatorname{Mod}-\mathcal{E}^{f} \rightarrow \operatorname{Mod}-\mathcal{E}_{g_{r}}^{f}$ la précomposition par $\mathcal{E}_{g_{r}}^{f} \rightarrow \mathcal{E}^{f} \quad(V, W) \mapsto V / W$.

- On note $\lambda: \operatorname{Mod}-\mathcal{E}_{\mathscr{g}_{r}}^{f} \rightarrow \operatorname{Mod}-\mathcal{E}^{f}$ la précomposition par le foncteur $\mathscr{E}^{f} \rightarrow \mathcal{E}_{\mathscr{g}_{r}}^{f}$ $V \mapsto(V, 0)$

- On désigne par $\omega: \operatorname{Mod}-\mathcal{E}_{g_{r}}^{f} \rightarrow \operatorname{Mod}-\mathcal{E}^{f}$ le foncteur défini par $\omega(X)(V)=$ $\bigoplus_{W \subset V} X(V, W)$ et le fait que $\omega(X)(f)$, pour $f: V \rightarrow V^{\prime}$ morphisme de $\mathcal{E}^{f}$, a pour composante $X(f): X\left(V^{\prime}, W^{\prime}\right) \rightarrow X(V, W)$ si $f(W)=W^{\prime}$ et 0 sinon (cf. proposition A.3). 
On remarque que l'on dispose d'un isomorphisme canonique $\omega(X \otimes \iota(F)) \simeq \omega(X) \otimes F$, où $X \in \mathrm{Ob}$ Mod- $\mathscr{E}_{\mathscr{g}_{r}}^{f}$ et $F \in \mathrm{Ob}$ Mod- $\mathcal{E}^{f}$. Par ailleurs, l'inclusion évidente de $X(V, 0)$ dans $\bigoplus_{W \subset V} X(V, W)$ définit une transformation naturelle $\lambda \hookrightarrow \omega$.

Le premier résultat d'homologie des foncteurs (sa démonstration établit clairement qu'il s'agit d'un résultat d'annulation) dont nous avons besoin pour établir le théorème principal de cet article est le suivant. Il s'agit de la variante en termes de groupes de torsion d'un cas particulier fondamental du théorème 10.2.1 de [9] (cf. aussi son corollaire 10.2.2).

ThÉORÈme D.2 (Djament). - Soient $X \in \operatorname{Mod}-\mathcal{E}_{\mathscr{g}_{r}}^{f}$ et $F$ un foncteur analytique de $\mathcal{F}$ (cf. définition 3.20). L'inclusion naturelle $\lambda(X) \hookrightarrow \omega(X)$ induit un isomorphisme

$$
\operatorname{Tor}_{*}^{\delta^{f}}(\lambda(X), F) \simeq \operatorname{Tor}_{*}^{\delta^{f}}(\omega(X), F) .
$$

Il revient au même de démontrer l'annulation de $\operatorname{Tor}_{*}^{\delta^{f}}(\omega(X) / \lambda(X), F)$. Quitte à remplacer $X$ par son quotient $X^{\prime}$ défini par $X^{\prime}(V, W)=X(V, W)$ si $W \neq 0$ et $X^{\prime}(V, 0)=0$, on est donc ramené à établir l'annulation de $\operatorname{Tor}_{*}^{\delta^{f}}(\omega(X), F)$ lorsque $\lambda(X)=0$. On s'appuie pour cela sur les lemmes suivants.

On commence par noter $\bar{P} \in \operatorname{Mod}-\mathcal{E}^{f}$ le noyau du morphisme d'augmentation $P_{k}^{\left(\delta^{f}\right)^{\mathrm{op}}} \rightarrow P_{0}^{\left(\delta^{f}\right)^{\mathrm{op}}}=\mathbb{k}$. Ainsi, les éléments $[l]-[0]$, où $l$ parcourt les formes linéaires non nulles sur $V$, forment une base de $\bar{P}(V)$. On a classiquement :

Lemme D.3. - Il existe un isomorphisme naturel $\operatorname{Tor}_{*}^{\mathcal{E}^{f}}(F \otimes \bar{P}, G) \simeq \operatorname{Tor}_{*}^{\mathcal{E}^{f}}(F, \Delta(G))$.

(On rappelle que le foncteur différence $\Delta$ est défini en 3.20.)

Ce lemme se déduit aussitôt du cas du degré 0 , par exactitude des foncteurs en jeu; la propriété provient alors de l'isomorphisme canonique $P_{V}^{\left(\delta^{f}\right)^{\mathrm{op}}} \otimes P_{W}^{\left(\delta^{f}\right)^{\mathrm{op}}} \simeq P_{V \oplus W}^{\left(\delta^{f}\right)^{\mathrm{op}}}$ en considérant une présentation projective de $F$.

Lemme D.4. - Si $X \in \operatorname{Mod}-\mathcal{E}_{g_{r}}^{f}$ vérifie $\lambda(X)=0$, il existe une suite exacte courte $0 \rightarrow Y \rightarrow X \otimes \iota(\bar{P}) \rightarrow X \rightarrow 0$ où $Y \in \operatorname{Mod}-E_{\mathscr{g} r}^{f}$ vérifie $\lambda(Y)=0$.

Démonstration. - On définit un morphisme $\iota(\bar{P}) \rightarrow \mathbb{k}$ en associant à $[l]-[0] \in \iota(\bar{P})(V, W)=\operatorname{Ker}\left(\mathbb{k}\left[V^{*}\right] \rightarrow \mathbb{k}\right) 0$ si la restriction de $l$ à $W$ est nulle et 1 dans le cas contraire. Ce morphisme est surjectif si $W$ est non nul. En le tensorisant par $X$, on obtient le résultat souhaité.

Démonstration du théorème D.2. - Par un argument de colimite, on peut supposer $F$ polynomial : il existe $d$ tel que $\Delta^{d}(F)=0$. On a vu qu'on peut également supposer $\lambda(X)=0$. En itérant $d$ fois l'épimorphisme du lemme D.4, on obtient une suite exacte $0 \rightarrow Y \rightarrow X \otimes \iota(\bar{P})^{\otimes d} \rightarrow X \rightarrow 0$ où $Y \in \operatorname{Mod}-\mathcal{E}_{\mathscr{g}_{r}}^{f}$ vérifie $\lambda(Y)=0$. Le foncteur $\omega$ étant exact, on en déduit une suite exacte $0 \rightarrow \omega(Y) \rightarrow \omega(X) \otimes \bar{P}^{\otimes d} \rightarrow \omega(X) \rightarrow 0$. La suite exacte longue d'homologie associée fournit, compte tenu de ce que $\operatorname{Tor}_{*}^{\mathcal{E}^{f}}\left(\omega(X) \otimes \bar{P}^{\otimes d}, F\right) \simeq \operatorname{Tor}_{*}^{\mathcal{E}^{f}}\left(\omega(X), \Delta^{d}(F)\right)=0$ (par le lemme D.3 appliqué $d$ fois), l'annulation de $\operatorname{Tor}_{0}^{\delta^{f}}(\omega(X), F)$ et des isomorphismes $\operatorname{Tor}_{i}^{\delta^{f}}(\omega(X), F) \simeq \operatorname{Tor}_{i-1}^{\delta^{f}}(\omega(Y), F)$. Par récurrence sur le degré homologique, le théorème s'ensuit. 
Le deuxième résultat d'homologie des foncteurs dont nous avons besoin dans cet article est le suivant. C'est une variante en termes de groupes de torsion du théorème A.8 de l'appendice de [14]; nous esquissons une démonstration se fondant sur le théorème D.2, en suivant [9], §13.2.

ThÉORÈme D.5 (Suslin). - Soient $A \in \mathrm{ObMod-} \mathcal{E}^{f}$ et $F \in \mathrm{Ob} \mathcal{F}$ analytique. Le morphisme canonique

$$
\operatorname{Tor}_{*}^{\varepsilon_{\text {inj }}^{f}}(\delta(A), \delta(F)) \stackrel{\simeq}{\longrightarrow} \operatorname{Tor}_{*}^{\varepsilon^{f}}(A, F)
$$

est un isomorphisme, où $\delta$ désigne le foncteur de restriction à la sous-catégorie $\mathcal{E}_{\mathrm{inj}}^{f}$ des injections de $\mathcal{E}^{f}$.

Lemme D.6. - Soient $X \in \mathrm{Ob}$ Mod- $\mathcal{E}_{\mathrm{inj}}^{f}$ et $F \in \mathrm{Ob} \mathcal{F}$. Il existe un isomorphisme naturel $\operatorname{Tor}_{*}^{\varepsilon_{\text {inj }}^{f}}(X, \delta(F)) \simeq \operatorname{Tor}_{*}^{\varepsilon^{f}}(\varpi(X), F)$, où $\varpi(X)$ est défini par $\varpi(X)(V)=\bigoplus_{W \subset V} X(V / W)$ et le fait que, pour toute application linéaire $f: V \rightarrow V^{\prime}, \varpi(X)(f): \varpi(X)\left(V^{\prime}\right) \rightarrow \varpi(X)(V)$ a pour composante $X\left(V^{\prime} / W^{\prime}\right) \rightarrow X(V / W)$ le morphisme induit par le monomorphisme $V / W \hookrightarrow V^{\prime} / W^{\prime}$ induit par $f$ si $f^{-1}\left(W^{\prime}\right)=W, 0$ sinon.

Démonstration. - L'isomorphisme en degré 0 se déduit facilement de la décomposition

$$
\operatorname{Hom}_{\mathcal{E}^{f}}(V,-) \simeq \coprod_{W \subset V} \operatorname{Hom}_{\mathcal{E}_{\mathrm{inj}}^{f}}(V / W,-)
$$

(qui traite le cas $F=P_{V}^{\delta^{f}}$ ). Le cas général s'en déduit par exactitude de $\varpi$ et $\delta$.

Lemme D.7. - Les endofoncteurs $\omega \kappa$ et $\varpi \delta$ de Mod- $\mathcal{E}^{f}$ sont isomorphes.

Démonstration. - On a $\omega \kappa(A)(V)=\varpi \delta(A)(V)=\bigoplus_{W \subset V} A(V / W)$, mais l'effet sur les morphismes n'est pas le même (l'un utilise l'image directe, l'autre l'image inverse d'un sous-espace vectoriel par une application linéaire). Néanmoins, on vérifie facilement (cf. [9], proposition 13.2.1 pour les détails) que l'application linéaire $\omega \kappa(A)(V) \rightarrow \varpi \delta(A)(V)$ ayant pour composante $A(V / W) \rightarrow A\left(V / W^{\prime}\right)$ le morphisme induit par la projection $V / W \rightarrow V / W^{\prime}$ lorsque $W \subset W^{\prime}$ et 0 sinon est un isomorphisme et qu'elle définit une transformation naturelle $\omega \kappa \rightarrow \varpi \delta$.

Démonstration du théorème D.5. - En utilisant successivement les lemmes D.6 et D.7 puis le théorème D.2, on obtient des isomorphismes naturels

$$
\operatorname{Tor}_{*}^{\varepsilon_{\text {inj }}^{f}}(\delta(A), \delta(F)) \simeq \operatorname{Tor}_{*}^{\delta^{f}}(\varpi \delta(A), F) \simeq \operatorname{Tor}_{*}^{\delta^{f}}(\omega \kappa(A), F) \simeq \operatorname{Tor}_{*}^{\mathcal{E}^{f}}(\lambda \kappa(A), F) ;
$$

on conclut en remarquant que $\lambda \kappa \simeq \mathrm{Id}$.

En fait, le théorème D.5 est valable dans un cadre beaucoup plus général : 
ThÉorème D.8 (Scorichenko). - Soient $\mathscr{G}$ une catégorie additive (essentiellement) petite et $\mathscr{C}_{\mathrm{inj}}$ la sous-catégorie des monomorphismes scindés de $\mathscr{G}$. Soient $F \in \mathrm{ObMod} \mathscr{\mathscr { C }}$ et $G \in \mathrm{Ob} \mathscr{C}$-Mod, $G$ étant supposé analytique ${ }^{(3)}$. Alors le morphisme canonique

$$
\operatorname{Tor}_{*}^{\ell_{\text {inj }}}(F, G) \rightarrow \operatorname{Tor}_{*}^{\mathscr{Q}}(F, G)
$$

est un isomorphisme (où l'on a, par abus, omis les foncteurs de restriction à $\mathscr{G}_{\mathrm{inj}}$ dans le membre de gauche).

La principale difficulté réside dans le fait que l'analogue du foncteur $\varpi$ qui apparaît dans le contexte général n'est ni explicite ni exact. Pour la démonstration, nous renvoyons à [27] (non publié), ou aux notes [10] qui en rendent disponibles les arguments.

Dans l'appendice F, nous utiliserons le cas particulier de ce théorème dans lequel $\mathscr{G}$ est la catégorie des modules projectifs de type fini sur un anneau fixé et $F$ un foncteur constant pour appliquer notre formalisme général à l'homologie des groupes linéaires.

\section{Appendice E}

\section{Les résultats de Betley sur les groupes symétriques revisités}

On conserve les notations de l'appendice $C$; notre anneau de base est $\mathbb{k}=\mathbb{Z}$. On note également $U: \Theta \rightarrow \Gamma$ le foncteur composé de l'inclusion de $\Theta$ dans la catégorie des ensembles finis et de $(-)_{+}$. On dispose donc de $U_{!}: \operatorname{Mod}-\Theta \rightarrow$ Mod- $\Gamma$ par la notation introduite à la proposition A.2.

On considère aussi la catégorie $\Sigma$ des ensembles finis avec bijections; les foncteurs d'inclusion $J^{\Omega}: \Sigma \rightarrow \Omega$ et $J^{\Theta}: \Sigma^{\text {op }} \rightarrow \Theta^{\text {op }}$ donnent lieu à $J_{!}^{\Omega}: \operatorname{Mod}-\Sigma \rightarrow \operatorname{Mod}-\Omega$ et $J_{!}^{\Theta}: \Sigma$-Mod $\rightarrow \Theta$-Mod.

Lemme E.1. - Pour E un ensemble fini, on a

$$
J_{!}^{\Theta}(F)(E)=\bigoplus_{E^{\prime} \subset E} F\left(E^{\prime}\right) .
$$

(On laisse au lecteur, dans cet énoncé comme dans la suite de l'appendice, le soin de préciser la fonctorialité en $E$, qui est analogue à plusieurs cas déjà rencontrés — cf. la proposition A.3.)

Démonstration. - Cela découle de l'isomorphisme canonique

$$
\left(J^{\Theta}\right)^{*}\left(P_{E}^{\Theta^{\mathrm{op}}}\right)=\mathbb{Z}\left[\operatorname{Hom}_{\Theta}(-, E)\right] \simeq \bigoplus_{E^{\prime} \subset E} \mathbb{Z}\left[\operatorname{Hom}_{\Sigma}\left(-, E^{\prime}\right)\right]=\bigoplus_{E^{\prime} \subset E} P_{E^{\prime}}^{\Sigma^{\mathrm{op}}}
$$

Proposition E.2. - La composée Mod- $\Theta \stackrel{U_{1}}{\longrightarrow} \operatorname{Mod}-\Gamma \stackrel{\mathrm{cr}}{\longrightarrow}$ Mod- $\Omega$ est isomorphe à Mod- $\Theta \stackrel{\left(J^{\Theta}\right)^{*}}{\longrightarrow} \operatorname{Mod}-\Sigma \stackrel{J_{!}^{\Omega}}{\longrightarrow}$ Mod- $\Omega$.

De surcroît, le foncteur $U_{!}$est exact.

\footnotetext{
(3) La définition est analogue à celle donnée dans le cadre de la catégorie $\mathcal{F}$. Le seul point à noter dans la définition de foncteur polynomial est qu'il faut imposer la nilpotence pour tous les foncteurs différences imaginables (avec un degré commun).

$4^{\text {e }}$ SÉRIE - TOME $43-2010-\mathrm{N}^{\mathrm{o}} 3$
} 
Démonstration. - Par un argument d'adjonction (ou plutôt sa variante en terme de produit tensoriel), la première assertion équivaut à l'existence d'un isomorphisme $U^{*} \circ i_{!} \simeq J_{!}^{\Theta} \circ\left(J^{\Omega}\right)^{*}$, qui résulte du lemme précédent.

Pour la deuxième assertion, il suffit de voir que $J_{!}^{\Omega}$ est exact, puisque cr est une équivalence de catégories ; cela provient d'une description explicite analogue à celle du lemme précédent (faisant intervenir les quotients au lieu des sous-ensembles d'un ensemble fini).

La suite spectrale du théorème suivant est équivalente à la conjonction des théorèmes 1.23 et $3.2 \mathrm{de}$ [5] (on rappelle que la naturalité du scindement suggérée par le théorème 1.23 semble incorrecte). On y note $F_{\infty}$ pour $\left(U^{*} F\right)_{\infty}$, et $\operatorname{cr}_{i}(F)$ pour $\operatorname{cr}(F)(\{1, \ldots, i\})$.

ThÉorème E.3 (Betley). - Pour tout $F \in \mathrm{Ob} \Gamma$-Mod, il existe des isomorphismes naturels

$$
H_{n}\left(\mathfrak{S}_{\infty} ; F_{\infty}\right) \simeq \bigoplus_{i \in \mathbb{N}} H_{n}\left(\mathfrak{S}_{\infty} \times \mathfrak{S}_{i} ; \operatorname{cr}_{i}(F)\right) \simeq \bigoplus_{\substack{p+q=n \\ i \in \mathbb{N}}} \operatorname{Tor}_{p}^{\mathfrak{S}_{i}}\left(H_{q}\left(\mathfrak{S}_{\infty} ; \mathbb{Z}\right), \operatorname{cr}_{i}(F)\right)
$$

(où l'action de $\mathfrak{S}_{i}$ sur $H_{q}\left(\mathfrak{S}_{\infty} ; \mathbb{Z}\right)$ est triviale), ainsi qu'une suite spectrale naturelle

$$
E_{p, q}^{2}=H_{p}\left(\mathfrak{S}_{\infty} ; H_{q}\left(\mathfrak{S}_{i} ; \mathrm{cr}_{i}(F)\right)\right) \Rightarrow H_{p+q}\left(\mathfrak{S}_{\infty} ; F_{\infty}\right)
$$

(où l'action de $\mathfrak{S}_{\infty}$ est triviale) qui s'effondre à la deuxième page et procure un isomorphisme non naturel

$$
H_{n}\left(\mathfrak{S}_{\infty} ; F_{\infty}\right) \simeq \bigoplus_{\substack{p+q=n \\ i \in \mathbb{N}}} H_{p}\left(\mathfrak{S}_{\infty} ; H_{q}\left(\mathfrak{S}_{i} ; \operatorname{cr}_{i}(F)\right)\right)
$$

Démonstration. - Les propositions E.2 et C.3 procurent des isomorphismes :

$$
\begin{aligned}
\operatorname{Tor}_{*}^{\Theta}\left(G, U^{*}(F)\right) & \simeq \operatorname{Tor}^{\Gamma}\left(U_{!}(G), F\right) \simeq \operatorname{Tor}_{*}^{\Omega}\left(\operatorname{cr} \circ U_{!}(G), \operatorname{cr}(F)\right) \\
& \simeq \operatorname{Tor}_{*}^{\Omega}\left(J_{!}^{\Omega} \circ\left(J^{\Theta}\right)^{*}(G), \operatorname{cr}(F)\right) \\
& \simeq \operatorname{Tor}_{*}^{\Sigma}\left(\left(J^{\Theta}\right)^{*}(G),\left(J^{\Omega}\right)^{*} \circ \operatorname{cr}(F)\right) \simeq \bigoplus_{i \in \mathbb{N}} \operatorname{Tor}_{*}^{\mathfrak{S}_{i}}\left(\left(J^{\Theta}\right)^{*}(G), \operatorname{cr}_{i}(F)\right) .
\end{aligned}
$$

On en déduit les isomorphismes annoncés à l'aide des propositions 2.22 et 2.26 . La suite spectrale et son effondrement proviennent de la proposition 2.27 .

Remarque E.4. - Notre méthode ne diffère pas fondamentalement de celle employée par Betley pour établir ce théorème, si l'on excepte le remplacement de la $K$-théorie stable généralisée par l'homologie de la catégorie $\Theta$.

\section{Appendice F}

\section{Un aperçu du cas des modules d'après Betley et Scorichenko}

Dans cet appendice, on se donne un anneau (unitaire) $A$, non nécessairement commutatif; on note $\mathcal{F}(A)$ pour $\mathbb{P}(A)$-Mod, où $\mathbb{P}(A)$ est la catégorie des $A$-modules à gauche projectifs de type fini. 
Comme dans l'exemple 1.9.4, $\mathbb{M}(A)$ désigne la sous-catégorie de $\mathbb{P}(A)$ avec les mêmes objets et les injections scindées comme morphismes. Nous aurons aussi besoin de la souscatégorie $\mathbb{E}(A)$ ayant les mêmes objets que $\mathbb{P}(A)$ et les épimorphismes pour flèches. La catégorie $\mathbb{E}(A)^{\text {op }}$ est équivalente à $\mathbb{M}\left(A^{\text {op }}\right)$ via le foncteur de dualité $\operatorname{Hom}_{A}(-, A)$.

On utilise également les notations $L_{q}$ et St introduites respectivement en 2.21 et en 2.18, le triplet $(\mathscr{C}, S, G)$ sous-jacent étant celui de l'exemple 1.9.4.

Lemme F.1. - Pour tout objet $V$ de $\mathbb{P}(A)$, il existe une suite spectrale du premier quadrant

$$
E_{p, q}^{2}=H_{p}\left(G L_{\infty}(A) ; H_{q}\left(\operatorname{Hom}_{A}(-, V)\right)_{\infty}\right) \Rightarrow L_{p+q}(V)
$$

où $\operatorname{Hom}_{A}(-, V)$ est vu comme foncteur de $\mathbb{E}(A)^{\text {op }}$ vers $\mathbf{A b}$.

Démonstration. - On note d'abord qu'on a $H_{q}\left(\operatorname{Hom}_{A}(-, V)\right)_{\infty} \simeq H_{q}\left(\operatorname{Hom}_{A}(-, V)_{\infty}\right)$ canoniquement puisqu'homologie et colimites filtrantes commutent.

Si $E$ est un $A$-module projectif de type fini, le stabilisateur de $V \hookrightarrow V \oplus E$ sous l'action de $G L(V \oplus E)$ s'identifie au produit semi-direct $\operatorname{Hom}_{A}(E, V) \rtimes G L(E)$. On en déduit aisément l'énoncé, en utilisant la suite spectrale de Lyndon-Hochschild-Serre.

Noter qu'il n'y a a priori aucune fonctorialité en $V$ sur cette suite spectrale : l'aboutissement est «naturellement» fonctoriel contravariant sur les monomorphismes scindés, tandis que le terme $E^{2}$ est «naturellement» fonctoriel covariant sur toutes les applications $A$-linéaires! En particulier, on ne semble pas disposer de description simple du morphisme canonique $L_{n}(V) \simeq H_{n}(\operatorname{St}(V)) \rightarrow L_{n}(0) \simeq H_{n}\left(G L_{\infty}(A)\right)$ (qui est induit par l'inclusion de $\operatorname{St}(V)$ dans $\left.G L_{\infty}(A)\right)$.

On a néanmoins le résultat suivant :

Lemme F.2. - Soit $n \in \mathbb{N}$. Supposons que, dans la suite spectrale précédente, le terme $E_{p, q}^{2}$ soit nul pour $p<n$ et $q>0$. Alors le foncteur $L_{n}$ est constant.

Démonstration. - Cette assertion provient des deux observations suivantes :

- l'épimorphisme de groupes $\pi: \operatorname{St}(V) \rightarrow G L_{\infty}(A)$ donné par la description précédente comme produit semi-direct induit, sous l'hypothèse d'annulation de l'énoncé, est un isomorphisme en homologie de degré au plus $n$, puisque $H_{*}(\pi)$ est le « coin » $L_{*}(V) \rightarrow E_{*, 0}^{2}$ (propriété générale de la suite spectrale de Lyndon-Hochschild-Serre);

- la composée du monomorphisme $G L_{\infty}(A) \hookrightarrow \operatorname{St}(V)$ (toujours donné par la description de $\operatorname{St}(V)$ de la démonstration du lemme précédent) avec l'inclusion $\operatorname{St}(V) \hookrightarrow G L_{\infty}(A)$ induit un isomorphisme en homologie (cf. la démonstration de la proposition 2.22), et sa composée avec l'épimorphisme $\pi$ est l'identité.

ThÉorème F.3 (Betley). - Si F est un foncteur polynomial de $\mathcal{F}(A)$ tel que $F(0)=0$, alors $H_{*}\left(G L_{\infty}(A), F_{\infty}\right)=0$.

Cette assertion est le théorème 4.2 de [3], où l'anneau $A$ est supposé commutatif, mais les arguments de Betley ne semblent en fait pas réellement requérir cette hypothèse (Betley suppose aussi $\mathbb{k}=\mathbb{Z}$, mais ce n'est pas restrictif). 
Démonstration. - On démontre l'assertion par récurrence sur le degré homologique. Si elle est vraie en degré $<n$, on a $E_{p, q}^{2}=0$ dans la suite spectrale du lemme F.1 pour $p<n$ et $q>0$, puisqu'alors $H_{q}\left(\operatorname{Hom}_{A}(-, V)\right)$ est un foncteur polynomial (car il est en fait exponentiel gradué par la formule de Künneth) nul en 0 (on applique ici l'hypothèse de récurrence à $A^{\mathrm{op}}$ ). On en déduit que $L_{i}$ est un foncteur constant pour $i \leq n$, en utilisant le lemme F.2. La suite spectrale du $\S 2.2$ et le théorème D.8 (dans le cas particulier où le foncteur contravariant est constant) donnent alors $H_{n}\left(G L_{\infty}(A) ; F_{\infty}\right)=0$ pour $F$ polynomial sans terme constant, d'où le théorème.

Remarque F.4. - Cette démonstration diffère profondément de celle de Betley. En effet, dans [3], il établit le résultat à partir de faits généraux sur la structure des foncteurs polynomiaux et surtout de théorèmes d'annulation démontrés dans son article antérieur [2]. Celui-ci se fondait sur des considérations explicites sur les groupes linéaires utilisant des arguments arithmétiques (différents selon qu'il s'agit de prouver l'annulation de la composante sans torsion de l'homologie ou de sa composante $p$-primaire pour un nombre premier $p$ ), qui permettent d'obtenir les annulations souhaitées, d'abord pour le cas crucial de $G L_{\infty}(\mathbb{Z})$. À l'inverse, la méthode ici suivie ne nécessite aucune considération d'ordre arithmétique, mais repose lourdement sur le théorème $\mathrm{D} .8$, résultat d'annulation tout à fait non trivial en homologie des foncteurs (même dans le cas particulier où le foncteur contravariant dans le groupe de torsion est constant).

\section{RÉFÉRENCES}

[1] J. BÉnAbou, Introduction to bicategories, in Reports of the Midwest Category Seminar, Springer, 1967, 1-77.

[2] S. Betley, Vanishing theorems for homology of $\mathrm{Gl}_{n}$ R, J. Pure Appl. Algebra 58 (1989), 213-226.

[3] S. BetLey, Homology of $\mathrm{Gl}(R)$ with coefficients in a functor of finite degree, J. Algebra 150 (1992), 73-86.

[4] S. Betley, Stable $K$-theory of finite fields, K-Theory 17 (1999), 103-111.

[5] S. Betley, Twisted homology of symmetric groups, Proc. Amer. Math. Soc. 130 (2002), 3439-3445.

[6] S. Betley, T. Pirashvili, Stable $K$-theory as a derived functor, J. Pure Appl. Algebra 96 (1994), 245-258 (electronic).

[7] M. Chalupnik, Koszul duality and extensions of exponential functors, Adv. Math. 218 (2008), 969-982.

[8] R. Charney, A generalization of a theorem of Vogtmann, in Proceedings of the Northwestern conference on cohomology of groups (Evanston, Ill., 1985), 44, 1987, 107-125.

[9] A. Duament, Foncteurs en grassmanniennes, filtration de Krull et cohomologie des foncteurs, Mém. Soc. Math. Fr. 111 (2007).

[10] A. Djament, Les résultats d'annulation homologique de Scorichenko, prépublication http://hal.archives-ouvertes.fr/hal-00411929/, 2009. 
[11] A. Dold, Zur Homotopietheorie der Kettenkomplexe, Math. Ann. 140 (1960), 278298.

[12] Z. Fiedorowicz, S. Priddy, Homology of classical groups over finite fields and their associated infinite loop spaces, Lecture Notes in Math. 674, Springer, 1978.

[13] V. Franjou, E. M. Friedlander, T. Pirashvili, L. Schwartz, Rational representations, the Steenrod algebra and functor homology, Panoramas et Synthèses 16, Soc. Math. France, 2003.

[14] V. Franjou, E. M. Friedlander, A. Scorichenko, A. Suslin, General linear and functor cohomology over finite fields, Ann. of Math. 150 (1999), 663-728.

[15] V. Franjou, J. Lannes, L. Schwartz, Autour de la cohomologie de Mac Lane des corps finis, Invent. Math. 115 (1994), 513-538.

[16] E. M. Friedlander, A. Suslin, Cohomology of finite group schemes over a field, Invent. Math. 127 (1997), 209-270.

[17] P. Gabriel, Des catégories abéliennes, Bull. Soc. Math. France 90 (1962), 323-448.

[18] P. Gabriel, M. Zisman, Calculus of fractions and homotopy theory, Ergebn. Math. Grenzg. 35, Springer New York, Inc., New York, 1967.

[19] C. Kassel, La K-théorie stable, Bull. Soc. Math. France 110 (1982), 381-416.

[20] N. J. Kunn, Computations in generic representation theory : maps from symmetric powers to composite functors, Trans. Amer. Math. Soc. 350 (1998), 4221-4233.

[21] J.-L. Loday, Cyclic homology, Grund. Math. Wiss. 301, Springer, 1998.

[22] A. PFISTER, Quadratic forms with applications to algebraic geometry and topology, London Mathematical Society Lecture Note Series 217, Cambridge Univ. Press, 1995.

[23] T. Pirashvili, Dold-Kan type theorem for Г-groups, Math. Ann. 318 (2000), 277-298.

[24] T. Pirashvili, Hodge decomposition for higher order Hochschild homology, Ann. Sci. École Norm. Sup. 33 (2000), 151-179.

[25] D. Quillen, On the cohomology and $K$-theory of the general linear groups over a finite field, Ann. of Math. 96 (1972), 552-586.

[26] W. Scharlau, Quadratic and Hermitian forms, Grund. Math. Wiss. 270, Springer, 1985.

[27] A. Scorichenko, Stable K-theory and functor homology over a ring, Thèse, Evanston, 2000.

[28] R. P. Stanley, Enumerative combinatorics. Vol. 1, Cambridge Studies in Advanced Math. 49, Cambridge Univ. Press, 1997.

[29] A. TouzÉ, Universal classes for algebraic groups, Duke Math. J. 151 (2010), 219-249.

[30] A. Touzé, Cohomology of classical algebraic groups from the functorial point of view, prépublication arXiv:0902.4459, à paraître dans Advances in Math.

[31] A. Touzé, W. VAn Der Kallen, Bifunctor cohomology and cohomological finite generation for reductive groups, Duke Math. J. 151 (2010), 251-278.

$4^{\mathrm{e}}$ SÉRIE - TOME $43-2010-\mathrm{N}^{\mathrm{O}} 3$ 
[32] A. Troesch, Quelques calculs de cohomologie de compositions de puissances symétriques, Comm. Algebra 30 (2002), 3351-3382.

[33] C. Vespa, Generic representations of orthogonal groups : the functor category $\mathscr{F}_{\text {quad }}$, J. Pure Appl. Algebra 212 (2008), 1472-1499.

(Manuscrit reçu le 5 janvier 2009; accepté, après révision, le 28 septembre 2009.)

\author{
Aurélien DJAMENT \\ CNRS et Laboratoire de mathématiques Jean Leray \\ Université de Nantes \\ Département de Mathématiques \\ 2 rue de la Houssinière \\ 44322 NANTES Cedex 03 \\ E-mail: aurelien.djament@univ-nantes.fr \\ Christine VEsPA \\ Institut de Recherche Mathématique Avancée \\ UMR 7501 \\ Université de Strasbourg et CNRS \\ 7 rue René Descartes \\ 67000 Strasbourg \\ E-mail: vespa@math.unistra.fr
}

